\title{
Fredholm Theory in Ordered Banach Algebras
}

\author{
by
}

Ronalda Abigail Marsha Benjamin

\author{
Dissertation presented for the degree of Doctor \\ of Philosophy in Mathematics in the Faculty of Science at \\ Stellenbosch University
}

Promoter: Prof. Sonja Mouton

March 2016 


\section{Declaration}

By submitting this dissertation electronically, I declare that the entirety of the work contained therein is my own, original work, that I am the sole author thereof (save to the extent explicitly otherwise stated), that reproduction and publication thereof by Stellenbosch University will not infringe any third party rights and that I have not previously in its entirety or in part submitted it for obtaining any qualification.

March 2016

Copyright (C) 2016 Stellenbosch University

All rights reserved 


\section{Abstract}

Since its inception, Fredholm theory has become an important aspect of spectral theory. Among the spectra arising within Fredholm theory is the Weyl spectrum which has been intensively studied by several authors, both in the operator case and in the general situation of Banach algebras.

The Weyl spectrum of a bounded linear operator $T$ on a Banach space $X$ is the set $\bigcap_{K \in \mathcal{K}(X)} \sigma(T+K)$, where $\sigma(T)$ denotes the spectrum of $T$ and $\mathcal{K}(X)$ the closed ideal of all compact operators on $X$. A recent result by $\mathrm{E}$. A. Alekhno shows that, if "Banach space" is replaced by an arbitrary complex Banach lattice $E$, then the Weyl spectrum of $T$ on $E$ can be made more precise, and takes on the form $\bigcap_{0 \leq K \in \mathcal{K}(E)} \sigma(T+K)$.

By an ordered Banach algebra (OBA) we mean a complex unital Banach algebra $A$ containing an algebra cone; that is, a subset $C$ which contains the unit of $A$ and is closed under addition, multiplication and positive scalar multiplication. As is well-known, the algebra of all bounded linear operators on a complex Banach lattice is an important example of an OBA.

If $A$ denotes an arbitrary OBA with algebra cone $C, B$ a Banach algebra and $T: A \rightarrow B$ a homomorphism with $\mathrm{N}(T)=\{a \in A: T a=0\}$ indicating the null space of $T$, then the Weyl spectrum $\bigcap_{c \in \mathrm{N}(T)} \sigma(a+c)$ of $a \in A$ is in general strictly contained in the set $\bigcap_{c \in C \cap N(T)} \sigma(a+c)$ - see Example 4.1.13. As a result of this, we investigate the latter set, which we shall refer to as the upper Weyl spectrum of $a \in A$. In this work the concept of the upper Browder spectrum of $a$ will also be introduced and results related to these spectra and the underlying sets of elements on which these spectra are defined will be given.

This thesis aims to present initial steps taken in the effort of unifying the theory of positivity in OBAs with the general Fredholm theory in Banach algebras. 


\section{Opsomming}

Sedert die bekendstelling daarvan, het die Fredholmteorie ' $n$ belangrike aspek van spektraalteorie geword. Onder die spektra wat ontstaan in Fredholmteorie is die Weyl spektrum, wat alreeds in diepte bestudeer is deur verskeie outeurs, beide in die operatorkonteks en in willekeurige Banach algebras.

Die Weyl spektrum van 'n begrensde lineêre operator $T$ op 'n Banach ruimte $X$ is die versameling $\bigcap_{K \in \mathcal{K}(X)} \sigma(T+K)$, waar $\sigma(T)$ die spektrum van $T$ voorstel en $\mathcal{K}(X)$ die geslote ideaal van kompakte operatore op $X$. 'n Resultaat wat onlangs deur E. A. Alekhno bewys is, toon dat, as "Banach ruimte" vervang word met 'n willekeurige Banach rooster $E$, dan kan die voorstelling van die Weyl spektrum van $T$ op $E$ meer presies gemaak word, en dit word gegee deur $\bigcap_{0 \leq K \in \mathcal{K}(E)} \sigma(T+K)$.

Met 'n geordende Banach algebra (GBA) bedoel ons 'n komplekse unitale Banach algebra $A$ wat 'n algebra-keël bevat; dit is, 'n deelversameling $C$ wat die eenheid van $A$ as element het en wat geslote is onder optelling, vermenigvuldiging en positiewe skalaarvermenigvuldiging. Die versameling van begrensde lineêre operatore op 'n komplekse Banach rooster is 'n belangrike voorbeeld van 'n GBA.

As $A$ 'n willekeurige GBA met algebra-keël $C$ voorstel, $B$ 'n Banach algebra en $T: A \rightarrow B$ 'n homomorfisme met $\mathrm{N}(T)=\{a \in A: T a=0\}$ die nulruimte van $T$, dan is die Weyl spektrum $\bigcap_{c \in \mathrm{N}(T)} \sigma(a+c)$ van $a \in A$ in die algemeen eg bevat in die versameling $\bigcap_{c \in C \cap N(T)} \sigma(a+c)$ - kyk na Voorbeeld 4.1.13. As gevolg hiervan, ondersoek ons die laasgenoemde versameling, wat ons die bo-Weyl spektrum van $a \in A$ sal noem. In hierdie werk word die konsep van die bo-Browder spektrum van $a$ ook bekend gestel en resultate wat verband hou met hierdie spektra en met die onderliggende versamelings van elemente waarop hierdie spektra gedefineer is sal gegee word.

Die doel van hierdie tesis is die bekendstelling van die beginstappe wat geneem is in die poging om die teorie van positiwiteit in GBAs met die algemene Fredholmteorie in Banach algebras te verenig. 


\section{Acknowledgements}

I wish to express my deep gratitude to my advisor Professor Sonja Mouton for her excellent guidance and support during the course of this study. To accomplish this project without her suggestions and commitment would not have been possible.

Thank you for the opportunity to study under your supervision. You have taught me a lot.

The financial assistance of the National Research Foundation (NRF) towards this research is hereby acknowledged. Opinions expressed and conclusions arrived at, are those of the author and are not necessarily to be attributed to the NRF.

This work was also financially supported by the Ernst and Ethel Eriksen Trust. Your financial assistance has made my dream a reality.

Many thanks to my examiners Professors P. Maritz, H. Raubenheimer and

S. Č. Živković-Zlatanović for the time and effort you invested in reviewing this dissertation. Your valuable comments and recommendations made this document look better.

To all my lecturers in the Mathematics Division at Stellenbosch University, I have greatly valued your (mathematical) input in my life. A special thank you to Professors A. Fransman, I. Rewitzky and L. Van Wyk and Dr. C. Naude for your caring words and advice throughout my studies.

Our department is filled with a group of wonderful postgraduate students who are both brilliant and funny. Our annual postgraduate outings will always be remembered. Thank you for the good times!

I wholeheartedly thank my parents for the countless sacrifices they have made to help me get to where I am today. Thank you also for your understanding during stressful times. Ek waardeer als wat julle doen: klein en groot.

I would also like to extend my sincere thanks to all the lovely people I knew and have come to know during my career as a graduate student at Stellenbosch University - you are too many to mention. I thank you for 
your love and words of encouragement that enabled me to see this journey through.

Thank you also to Professor Toit Mouton for inviting me numerous times to eat some delicious food.

Last but not least, my heartfelt appreciation goes to the Almighty God for the privilege of education and for enabling me to come this far.

"But by the grace of God I am what I am..." 1 Corinthians 15:10 


\section{Dedication}

To my father on the occasion of his seventieth birthday. 


\section{Contents}

Declaration $\quad$ i

$\begin{array}{ll}\text { Abstract } & \text { ii }\end{array}$

Opsomming

Acknowledgements

Dedication vi vi vin

$\begin{array}{ll}\text { List of notations } & \text { ix }\end{array}$

Introduction $\quad$ xiii

1 Preliminaries 1

1.1 Banach algebra theory ................ 1

1.2 Spectral theory in Banach algebras . . . . . . . . . . . . . . 4

1.3 Functional Calculus . . . . . . . . . . . . . . . . 8

1.4 Ideals in Banach algebras . . . . . . . . . . . . . . 9

1.5 Fredholm theory in Banach algebras . . . . . . . . . . . 11

1.6 Banach lattice theory . . . . . . . . . . . . . . . . . . 13

1.7 Ordered Banach algebras . . . . . . . . . . . . . . . 16

1.8 Spectral theory in OBAs . . . . . . . . . . . . . 18

1.9 Irreducibility in OBAs . . . . . . . . . . . . 20

2 Poles and the Laurent expansion of the resolvent 28

2.1 Ideals, homomorphisms and poles . . . . . . . . . . . . . 28

2.2 Coefficients of Laurent series . . . . . . . . . . . . . . . . . . 31

3 Upper Browder and upper Weyl elements 35 
3.1 Definitions and examples . . . . . . . . . . . . 36

3.2 Examining the equation $\mathcal{W}_{\mathrm{T}}=\mathcal{W}_{\mathrm{T}}^{+} \ldots \ldots \ldots 39$

3.3 Basic properties of upper Browder and upper Weyl elements . 49

3.4 Perturbation results . . . . . . . . . . . . . . . . . . 54

3.5 Regularities .................... 56

4 The upper Browder and upper Weyl spectra 59

4.1 Elementary properties and examples . . . . . . . . . . . . . 59

4.2 Spectral mapping theorems . . . . . . . . . . . . . 66

4.3 Connected hulls . . . . . . . . . . . . . . . . . . 69

5 The upper Browder spectrum property 72

5.1 Introduction . . . . . . . . . . . . . . . 72

5.2 Finite-dimensional semisimple OBAs . . . . . . . . . . . 75

5.3 Homomorphisms with closed range having the Riesz property 80

5.4 Homomorphisms having the strong Riesz property . . . . . . 84

5.5 OBAs with disjunctive products . . . . . . . . . . . 86

6 The lower Weyl and Lozanovsky spectra 92

6.1 Introducing the lower Weyl spectrum . . . . . . . . . . . . . . . 92

6.2 A note on the Lozanovsky spectrum . . . . . . . . . . . . . 98

$\begin{array}{ll}\text { Conclusion } & 104\end{array}$

$\begin{array}{ll}\text { List of references } & 106\end{array}$

$\begin{array}{ll}\text { Index } & 109\end{array}$ 


\section{List of notations}

Throughout the dissertation, we shall adhere to the following notations.

\section{Operations}

$\|a\| \quad$ norm of normed space element $a$

$\|T\|_{r} \quad r$-norm of a regular operator $T$

$a \circ b \quad$ quasi-product of $a$ and $b$ or composition of functions $a$ and $b$

$x_{\alpha} \downarrow x \quad$ decreasing net $\left(x_{\alpha}\right)$ satisfying $\inf \left\{x_{\alpha}\right\}=x$

$x_{\alpha} \uparrow x \quad$ increasing net $\left(x_{\alpha}\right)$ satisfying $\sup \left\{x_{\alpha}\right\}=x$

$f_{\mid X} \quad$ restriction of a function $f$ to a set $X$

$\operatorname{dim} B \quad$ dimension of a vector space $B$

$\bigoplus_{i=1}^{n} B_{i}$ direct sum of the sets $B_{i}$

$\eta K \quad$ connected hull of a compact set $K$

$\partial K \quad$ topological boundary of a compact set $K$

\section{Sets}

$\mathbb{R}(\mathbb{C}) \quad$ set of real (complex) numbers

$\mathbb{R}^{n}\left(\mathbb{C}^{n}\right) \quad$ set of $n$-tuples $(n \geq 2)$ with real (complex) entries

$\left(\mathbb{R}^{n}\right)^{+} \quad$ set of $n$-tuples $(n \geq 1)$ with non-negative real entries

$\mathbb{D} \quad$ closed unit disc in $\mathbb{C}$

$H(\Omega) \quad$ algebra of complex-valued functions holomorphic on $\Omega \subseteq \mathbb{C}$

$\bar{B}_{A} \quad$ closure of a set $B$ in a metric space $A$

span $B \quad$ linear span of a set $B$ in a set $A$

$V_{+} \quad$ positive cone in a vector lattice $V$

$V_{n}^{\sim} \quad$ order continuous dual of a vector lattice $V$

$B^{d} \quad$ disjoint complement of a subset $B$ of a vector lattice

$\operatorname{Comm}(a)$ commutant of an algebra element $a$ 
$\operatorname{Comm}^{2}(a)$ double commutant of an algebra element $a$

$A_{1} \quad$ unitization of a Banach algebra $A$

$A_{n} \quad$ set of all order continuous elements of an OBA $A$

$\mathrm{OI}(A) \quad$ set of order idempotents of an OBA $A$

$A^{-1} \quad$ set of invertible elements of a unital algebra $A$

$A^{D} \quad$ set of generalized Drazin invertible elements of an algebra $A$

$q-A^{-1} \quad$ set of quasi-invertible elements of an algebra $A$

$\mathrm{QN}(A) \quad$ set of quasinilpotent elements of a Banach algebra $A$

$\mathrm{N}(T) \quad$ null space of a linear operator $T$

$\mathcal{F}_{T} \quad$ set of Fredholm elements relative to $T$

$\mathcal{B}_{T} \quad$ set of Browder elements relative to $T$

$\mathcal{B}_{T}^{+} \quad$ set of upper Browder elements relative to $T$

$\mathcal{W}_{T} \quad$ set of Weyl elements relative to $T$

$\mathcal{W}_{T}^{+} \quad$ set of upper Weyl elements relative to $T$

\section{Ideals}

$\operatorname{Rad}(A)$ radical of an algebra $A$

$\mathcal{F}(X) \quad$ ideal of finite-rank operators on a Banach space $X$

$\mathcal{K}(X) \quad$ ideal of compact operators on a Banach space $X$

$\mathcal{K}^{r}(E) \quad$ ideal of $r$-compact operators on a Banach lattice $E$

\section{Spaces}

$E_{\mathbb{R}} \quad$ real Banach lattice

$C(K)$ algebra of continuous complex-valued functions on a compact set K

$\mathscr{A}(\mathbb{D})$ disc algebra

$M_{n}(A)$ algebra of $n \times n$ matrices with entries in an algebra $A$

$M_{n}^{u}(A)$ algebra of upper triangular matrices in $M_{n}(A)$

$M_{n}^{l}(A)$ algebra of $n \times n$ lower triangular matrices in $M_{n}(A)$

$\mathcal{L}(X)$ algebra of bounded linear operators on a Banach space $X$

$\mathcal{L}^{r}(E)$ algebra of regular operators on a Banach lattice $E$

$\mathcal{Z}(E)$ algebra of central operators on a Banach lattice $E$ 
$l^{\infty}(A)$ algebra of norm bounded sequences of elements of an algebra $A$

$l^{2}(A)$ algebra of square-summable sequences with entries in an algebra A

\section{Spectra}

$\sigma(a) \quad$ spectrum of a Banach algebra element $a$

$\sigma^{\prime}(a)$ non-zero spectrum of a Banach algebra element $a$

iso $\sigma(a)$ set of isolated points of $\sigma(a)$

acc $\sigma(a)$ set of accumulation points of $\sigma(a)$

$\rho(a) \quad$ resolvent set of a Banach algebra element $a$

$r(a)$ spectral radius of a Banach algebra $a$

$D(a, I)$ the set of all $\lambda$ in $\sigma(a)$ which are not Riesz points of $\sigma(a)$ w.r.t. $I$

$\sigma_{o}(T) \quad o$-spectrum of $T \in \mathcal{L}^{r}(E)$

$\sigma_{e}(T) \quad$ essential spectrum of $T \in \mathcal{L}(E)$

$\sigma_{o e}(T)$ order essential spectrum of $T \in \mathcal{L}^{r}(E)$

$\sigma_{e l}(T)$ Lozanovsky essential spectrum of a positive operator $T$

$\beta_{T}(a) \quad$ Browder spectrum of a Banach algebra element $a$ w.r.t. $T$

$\beta_{T}^{+}(a) \quad$ upper Browder spectrum of a Banach algebra element $a$ w.r.t. $T$

$\omega_{T}(a)$ Weyl spectrum of a Banach algebra element $a$ w.r.t. $T$

$\omega_{T}^{+}(a)$ upper Weyl spectrum of a Banach algebra element $a$ w.r.t. $T$

$\omega_{T}^{-}(a)$ lower Weyl spectrum of a Banach algebra element $a$ w.r.t. $T$

$\omega_{T}^{L}(a) \quad$ Lozanovsky spectrum of a Banach algebra element $a$ w.r.t. $T$

\section{Elements}

$\mathbf{1}_{A}$ unit of an algebra $A$

$a_{p} \quad$ the element pap

$p^{d} \quad$ the element $\mathbf{1}-p$

$P_{B} \quad$ order projection on the projection band $B$

$T_{\mathbb{C}} \quad$ the operator $T+i 0$

$a^{-1} \quad$ inverse of an algebra element $a$

$a^{D} \quad$ generalized Drazin inverse of a Banach algebra element $a$

$x^{+} \quad$ positive part of a vector lattice element $x$ 
$x^{-} \quad$ negative part of a vector lattice element $x$

$|x| \quad$ modulus of a vector lattice or OBA element $x$

$\sup \{x, y\}$ supremum of vector lattice or OBA elements $x$ and $y$

$\inf \{x, y\}$ infimum of vector lattice or OBA elements $x$ and $y$

$p(a, \lambda) \quad$ spectral idempotent of $a$ corresponding to $\lambda \in$ iso $\sigma(a)$ 


\section{Introduction}

This thesis has as its main motivation that of unifying the theory of positivity in ordered Banach algebras with the general Fredholm theory in Banach algebras.

An important class of operators which occur within the classical Fredholm theory is the class of Weyl operators. There are a number of equivalent ways in which one can define a Weyl operator on a Banach space. According to one characterization, an operator $S$ on a Banach space $X$ is called Weyl if $S$ can be written as a sum of an invertible operator and a compact operator on $X$. In his 2007 paper [3], by primarily focussing on the spectrum which arises from the class of Weyl operators, E. A. Alekhno essentially asks in the case where $X$ is ordered by some relation (in particular when $X$ is a Banach lattice) - to what extent the element of "positivity" has an effect on certain results within the classical Fredholm theory. Remarkably, it turns out that an operator $S$ on a (complex) Banach lattice $E$ is Weyl if and only if $S$ can be decomposed as a sum of an invertible operator and a positive compact operator on $E$ ([4], Theorem 3). Alekhno's discovery demonstrates a strong relation between the theory of positive operators on Banach lattices and the classical Fredholm theory.

The primary structure for us will be a Banach algebra. All Banach algebras considered are assumed to be complex and unital.

In 1982 R. E. Harte showed, in light of a theorem of F. V. Atkinson ([12], p.4), that homomorphisms between Banach algebras gave rise to an abstract version of Fredholm theory. We recall the following definition.

Definition 0.0.1. ([20], p.431) Let $A$ and $B$ be Banach algebras and $T: A \rightarrow B$ be a homomorphism. An element $a \in A$ is called

(i) Fredholm if $\mathrm{Ta} \in B^{-1}$,

(ii) Weyl if there exist elements $b \in A^{-1}$ and $c \in \mathrm{N}(T)$ such that $a=b+c$,

(iii) Browder if there exist commuting elements $b \in A^{-1}$ and $c \in \mathrm{N}(T)$ such that $a=b+c$, 
where $A^{-1}$ denotes the set of invertible elements of $A$ and the null space of $T$ is indicated by $\mathrm{N}(T):=\{a \in A: T a=0\}$.

We point out that the classical Fredholm theory for operators on a Banach space $X$ corresponds to the Fredholm theory of the Banach algebra $\mathcal{L}(X)$ of bounded linear operators on $X$ relative to the canonical homomorphism $\pi$ : $\mathcal{L}(X) \rightarrow \mathcal{L}(X) / \mathcal{K}(X)$, where $\mathcal{K}(X)$ denotes the ideal of compact operators on $X$. Recalling Alekhno's discovery mentioned at the end of the second paragraph, the identity

$$
\mathcal{L}(E)^{-1}+\mathrm{N}(\pi)=\mathcal{L}(E)^{-1}+(K \cap \mathrm{N}(\pi)),
$$

where $K$ indicates the cone of positive operators on $E$, holds.

By an ordered Banach algebra (we abbreviate it as OBA) - which we shall denote by $(A, C)$ - we mean a Banach algebra $A$ containing an algebra cone; that is, a subset $C$ which contains the unit of $A$ and is closed under addition, multiplication and positive scalar multiplication. As is well-known, the algebra of all bounded linear operators on a Banach lattice is an important example of an OBA.

Since OBAs were introduced by H. Raubenheimer and S. Rode in [34], several problems which originated in $\mathcal{L}(E)$ have been investigated in an OBA context. On the other hand, new insights established in this more general setting could be applied back to $\mathcal{L}(E)$ and various other examples of OBAs.

In view of (0.0.2) one is tempted to ask the following question:

Question 0.0.3. If $(A, C)$ denotes an arbitrary $O B A$ and $T$ a homomorphism from $A$ to a general Banach algebra $B$, is it true that

$$
A^{-1}+\mathrm{N}(T)=A^{-1}+(C \cap \mathrm{N}(T)) ?
$$

According to Example 3.1.8 the answer to the above-stated question is negative for a general OBA. Consequently, we make the following definition:

Definition 0.0.5. Let $(A, C)$ be an $O B A$ and $T$ be a homomorphism from $A$ to a general Banach algebra $B$. An element $a \in A$ is called

(i) upper Weyl if there exist elements $b \in A^{-1}$ and $c \in C \cap \mathrm{N}(T)$ such that $a=b+c$, 
(ii) upper Browder if there exist commuting elements $b \in A^{-1}$ and $c \in C \cap \mathrm{N}(T)$ such that $a=b+c$.

Evidently, the above definition provides a means by which positivity theory in OBAs is connected with the abstract Fredholm theory. Clearly:

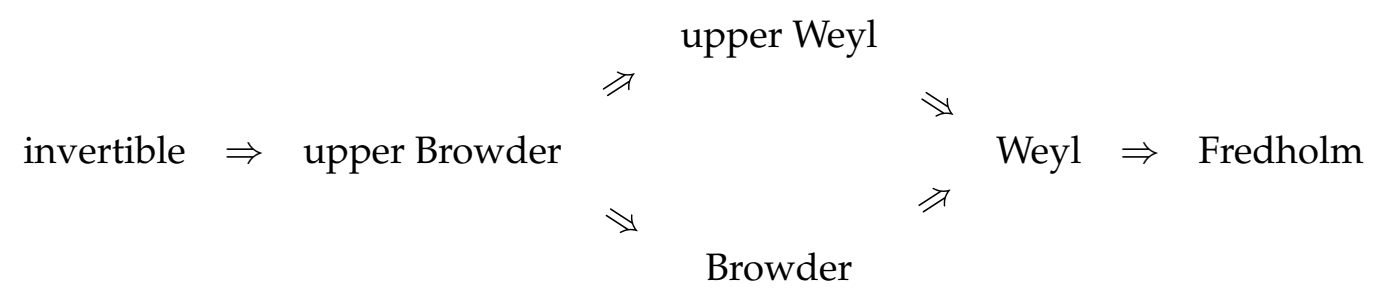

This thesis consists of six chapters. We give a short introduction to what is studied in each chapter:

In Chapter 1 we review some basic concepts and establish the terminology and notation needed throughout the rest of this thesis. This chapter is fairly lengthy since the treatment is self-contained. In particular, we point out that Section 1.9 is quite long (this is done for the reader's convenience) as the theory developed in this section is relatively new in the context of OBAs.

Chapter 2 can be viewed as a repository chapter in which we display a number of observations that will be employed in the rest of the document. In Section 2.1, in particular, we gather new insights regarding ideals, homomorphisms and poles that stem from the preliminary theory. As will be seen, Section 2.2 contains several results (whose proofs rely mainly on the holomorphic functional calculus) giving useful properties of the coefficients of the main part of the Laurent series of the resolvent.

Throughout the discussion that follows, let $(A, C)$ denote an $O B A$ and $T$ be a homomorphism from $A$ to a general Banach algebra $B$.

In Chapter 3 we develop the theory of upper Weyl and upper Browder elements in OBAs. We shall begin this chapter with the definitions of upper Weyl and upper Browder elements as introduced in Definition 0.0.5 and provide a selection of examples illustrating that the converse implications in the implication-scheme above generally do not hold. As a follow up (in view of (0.0.2)), we identify in Section 3.2 classes of homomorphisms $T$ relative to which every Weyl element can be decomposed as a sum of an invertible element and a positive element in $\mathrm{N}(T)$. Our main result in this 
section is Theorem 3.2.6(a), which states that the sets of Weyl and upper Weyl elements coincide relative to $T$ having the Riesz property and satisfying $\mathrm{N}(T)=\operatorname{span}(C \cap \mathrm{N}(T))$. This result can be viewed as a generalization of (0.0.2) in the case where the Banach lattice is either an $A L$ - or an $A M$ space.

How different the classes of upper Weyl and upper Browder elements are to the sets of Weyl and Browder elements, respectively, becomes more evident when studying some of their fundamental arithmetic properties in Section 3.3. Nevertheless, under the additional assumption that the homomorphism has the Riesz property, we find that certain algebraic properties known to hold for Weyl and Browder elements are inherited by upper Weyl and upper Browder elements, respectively (see, for instance, Lemma 3.3.4 and Theorem 3.3.8). In Section 3.4 we study the behaviour of upper Weyl and upper Browder elements under perturbation by elements from a number of sets. Among other things, we establish that the upper Weyl elements remain stable under perturbation by both positive and negative elements in the null space of a homomorphism which has the Riesz property (Proposition 3.4.1). In conclusion, we study in Section 3.5 regularities in connection with upper Weyl and upper Browder elements.

In Chapter 4 special attention is given to the corresponding spectra derived from the sets of upper Weyl and upper Browder elements. In particular, we focus on some aspects closely related to spectral theory: spectral mapping theorems and the relationship between the connected hulls of different spectra.

Suppose that $\sigma(\mathrm{Ta}), \omega_{T}(a), \beta_{T}(a)$ and $\sigma(a)$ denote, respectively, the Fredholm, Weyl, Browder and (usual) spectra of a Banach algebra element $a$. The sets of upper Weyl and upper Browder elements give (in a natural way) rise to two new spectra, defined for elements of a general OBA: the upper Browder spectrum of $a \in A$ is given by

$$
\beta_{T}^{+}(a)=\{\lambda \in \mathbb{C}: \lambda \mathbf{1}-a \text { is not upper Browder }\}=\bigcap_{\substack{c \in \mathcal{C} \cap N(T) \\ a c=c a}} \sigma(a+c)
$$

and the upper Weyl spectrum of $a \in A$ is given by

$$
\omega_{T}^{+}(a)=\{\lambda \in \mathbb{C}: \lambda \mathbf{1}-a \text { is not upper Weyl }\}=\bigcap_{c \in C \cap N(T)} \sigma(a+c) .
$$


These spectra are non-empty and compact subsets of the complex plane (Corollary 4.1.12). However, as suggested by Example 4.1.2, one should be careful to replace the element $\lambda \mathbf{1}-a$ in the definitions of $\beta_{T}^{+}(a)$ and $\omega_{T}^{+}(a)$ by $a-\lambda \mathbf{1}$. We point out that the replacement becomes possible whenever $T$ satisfies the Riesz property (Proposition 4.1.3).

Evidently, in view of the implication-scheme displayed before, we have (for $a \in A$ ) that

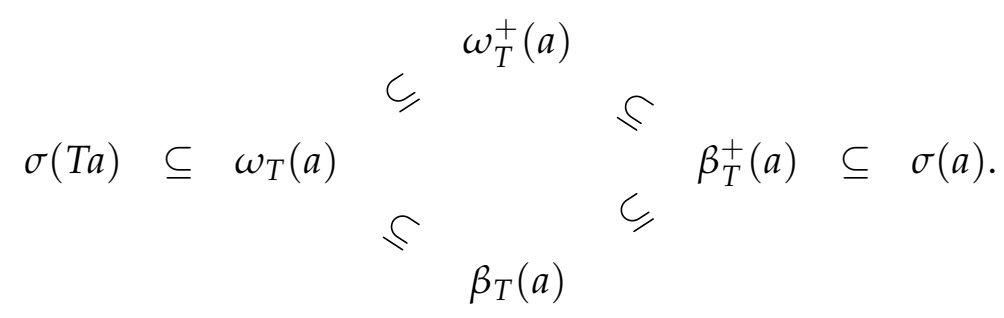

An important insight is revealed by Example 4.2.2, namely that, under the conditions developed by Harte (Theorem 4.2.1) which guarantee oneway spectral mapping theorems for both the Weyl and Browder spectra, the upper Weyl and upper Browder spectra do not satisfy the given one-way spectral inclusions. Up to now, we have only managed to set up a spectral inclusion result for the upper Weyl and upper Browder spectra dealing with a special holomorphic function, to be specific, the inverse function $\lambda \mapsto \frac{1}{\lambda}$ (see Proposition 4.2.5).

A result due to $\mathrm{H}$. Mouton, $\mathrm{S}$. Mouton and $\mathrm{H}$. Raubenheimer states that the connected hulls of the Fredholm, Weyl and Browder spectra coincide relative to Banach algebra homomorphisms with closed range having the Riesz property (Theorem 1.5.8). Under the latter assumptions on the homomorphism, we show in Theorem 4.3.2 that the connected hulls of the Fredholm, Weyl, Browder and upper Weyl spectra of all elements $a$, with the property that

$$
p(a, \lambda) \in \operatorname{span}(C \cap \mathrm{N}(T)) \text { for all } \lambda \in(\text { iso } \sigma(a)) \backslash \sigma(T a),
$$

where $p(a, \lambda)$ denotes the spectral idempotent of $a$ corresponding to an isolated point $\lambda$ of $\sigma(a)$, coincide. A simple example illustrates that the connected hull of the upper Browder spectrum cannot in general be added to the list of identities in Theorem 4.3.2 (see Example 4.3.3).

In order to introduce Chapter 5, we make the following assumptions.

The following assumptions are valid throughout the discussion that follows: $E$ is a complex Banach lattice, $S$ is a positive operator on $E$ and $\pi: \mathcal{L}(E) \rightarrow$ 
$\mathcal{L}(E) / \mathcal{K}(E)$ is the canonical homomorphism on $\mathcal{L}(E)$.

It is known that the spectral radius of a positive operator on a Banach lattice belongs to the spectrum of the operator. Prior to his discovery that the Weyl and upper Weyl spectra of a bounded linear operator coincide, Alekhno investigated the following question in [3].

Problem 1: If $r(S) \notin \sigma_{e}(S)$, where $\sigma_{e}(S)=\sigma(\pi S)$ denotes the essential spectrum of $S$, do we have that $r(S) \notin \omega_{\pi}^{+}(S)$ ?

The above problem in the context of OBAs becomes:

Problem 2: If $a \in C$ is such that $r(a) \notin \sigma(T a)$, do we have that $r(a) \notin \omega_{T}^{+}(a)$ ? Accordingly, in view of Theorem 4.3.2 and Example 4.3.3, a more general question is: If $a \in C$ is such that $r(a) \notin \sigma(T a)$, does it follow that $r(a) \notin$ $\beta_{T}^{+}(a)$ ? An element satisfying this condition is said to have the upper Browder spectrum property (relative to $T$ ).

It is not yet known whether all positive operators on arbitrary Banach lattices have the upper Browder spectrum property (relative to $\pi$ ).

The central problem of Chapter 5 is to present different types of sufficient conditions for a positive element to have the upper Browder spectrum property. In view of Theorem 4.3.2, we shall be concerned with homomorphisms with closed range satisfying the Riesz property.

First we consider the finite-dimensional case (Section 5.2). Indeed, an application of the Wedderburn-Artin Theorem ([10], Theorem 2.1.2), enables us to show that any finite-dimensional semisimple OBA is algebraically isomorphic to an OBA in which all positive elements have the upper Browder spectrum property (see Corollary 5.2.11).

In the rest of our discussion on Chapter 5 we consider an element $a \in C$ satisfying $r(a) \notin \sigma(T a)$.

In Section 5.3 we study infinite-dimensional OBAs. Using the spectral mapping theorem, it is straightforward to show that $r(a) \notin \sigma(a+p(a, r(a))$ (Corollary 2.2.6), and therefore $r(a) \notin \beta_{T}^{+}(a)$ whenever $p(a, r(a)) \in C$ (Theorem 5.3.3). This fact generalizes ([3], Theorem 4(a)), where the author used totally different methods for positive operators on Banach lattices. Also, this observation (under natural conditions on the algebra cone) has the following three applications:

(1) All positive elements of commutative semisimple OBAs have the upper 
xix

Browder spectrum property.

(2) All positive elements of semisimple OBAs with inverse-closed algebra cones have the upper Browder spectrum property.

(3) All positive operators in the center of a Banach lattice have the upper Browder spectrum property.

We proceed to show in Section 5.4 that all results obtained in Section 5.3 can be extended to homomorphisms having the strong Riesz property.

Over the last twenty years the knowledge on the spectral theory of positive OBA elements has increased considerably. Recent work done by Alekhno in [6] shows that, under natural conditions, the spectrum of a positive element is determined by the spectra of irreducible elements. The notion of an irreducible OBA element is also introduced in [6], where it is established that these elements have useful spectral properties. The ideas drawn from Alekhno's work open doors to the study of whether positive elements of arbitrary Dedekind complete semisimple OBAs with disjunctive products have the upper Browder spectrum property. Our main result here is Theorem 5.5.4, where we show that, under certain circumstances, the spectral radius of $a$ is not in the upper Browder spectra of the elements $q_{i} a q_{i}$, where the terms $q_{i}$ are certain idempotents satisfying $0 \leq q_{i} \leq \mathbf{1}$. Unfortunately, at this stage, it is not possible to say more; that is, we are unable to replace, in general, the union of the upper Browder spectra of $q_{i} a q_{i}$ in Theorem 5.5.4 by $\beta_{T}^{+}(a)$. This section is closed by applying Theorem 5.5.4 to the positive regular operators on a Dedekind complete Banach lattice (Corollary 5.5.8, see also Corollary 5.5.9).

We conclude this thesis with Chapter 6 where some results involving the lower Weyl and Lozanovsky spectra for an arbitrary positive OBA element are presented. These spectra are natural generalizations of the lower Weyl and Lozanovsky essential spectra for a positive bounded linear operator on a Banach lattice. The two questions, which originated in $\mathcal{L}(E)$, that are addressed in this chapter are the following:

(i) Given that the spectral radius of a positive element is outside its Fredholm spectrum, what conditions suffice for it to be outside the lower Weyl spectrum of the element?

(ii) When does the Lozanovsky spectrum contain the Weyl spectrum?

In [3] and [4] Alekhno identified conditions on a positive bounded linear operator $S$ (and the Banach lattice on which $S$ acts) which ensure that (i) and 
(ii) hold; these proofs in the operator-context relied heavily on operator and lattice theoretic arguments. Our aim in this chapter is to present solutions in the OBA-context whose proofs use basic techniques available in the abstract setting of an OBA. The examination of (i) takes place in Section 6.1, while we investigate (ii) in Section 6.2. 


\section{Chapter 1}

\section{Preliminaries}

This chapter is a collection of basic definitions, notations and results that will be useful in the remaining chapters. The results for which proofs are provided are either new ideas or facts that were given in an article without proof.

\subsection{Banach algebra theory}

This section contains a brief look into the theory of Banach algebras.

Definition 1.1.1 (Algebra). ([24], p.394) An algebra is a vector space A over a field $K$ such that for each ordered pair of elements $x, y \in A$, a unique product $x y \in A$ is defined satisfying the properties

- $x(y z)=(x y) z$

- $(x+y) z=x z+y z$

- $x(y+z)=x y+x z$

- $\lambda(x y)=(\lambda x) y=x(\lambda y)$

for all $x, y, z \in A$ and $\lambda \in K$.

If $K=\mathbb{R}$ in Definition 1.1.1, then $A$ is called a real algebra, whereas if $K=\mathbb{C}$, then $A$ is said to be a complex algebra. Hereafter we use the word "algebra" to mean "complex algebra".

We say that algebra elements $a$ and $b$ commute whenever $a b=b a$. An algebra $A$ is then said to be commutative if any two of its elements commute. For an element $a$ in an algebra, the commutant of $a$, being the set of all 
elements which commute with $a$, is denoted by $\operatorname{Comm}(a)$. The double commutant of $a$, denoted by $\operatorname{Comm}^{2}(a)$, is the set of all elements that commute with every element in $\operatorname{Comm}(a)$.

An algebra $A$ is said to be unital if it has a unit, which we will denote by $\mathbf{1}_{A}$. If the algebra under discussion is clear from the context we will only write 1. If $A$ is a unital algebra, then $a \in A$ is said to be invertible if there exists an element $b \in A$ satisfying $a b=\mathbf{1}=b a$. If such $b$ exists, then it is unique. We will refer to $b$ as the inverse of $a$ and denote it by $a^{-1}$. We write $A^{-1}$ for the set of all invertible elements of $A$.

Definition 1.1.2 (Banach algebra). ([10], Definition p.30) An algebra $A$ is said to be a Banach algebra if $A$ is a Banach space for a norm $\|\cdot\|$ and satisfies $\|a b\| \leq$ $\|a\|\|b\|$ for all $a, b \in A$.

It is known that, for a Banach algebra $A$, the set $A^{-1}$ is open. We may always assume that the unit of a unital Banach algebra has norm 1 ([10], p. 30). We remark that, since almost all results in this thesis apply to unital Banach algebras (or unital algebras), from this point on we will only write "Banach algebra" ("algebra") to mean "unital Banach algebra" ("unital algebra"). In cases where the results for Banach algebras (or just algebras) without units are stated, this will be explicitly mentioned.

A simple example of a Banach algebra is the complex plane $\mathbb{C}$, where the norm is given by the modulus function. The space $C(K)$ of all continuous complex-valued functions defined on a compact set $K$ equipped with pointwise addition and multiplication and the sup-norm $\|f\|=\sup \{|f(z)|: z \in$ $K\}$ is an example of a commutative Banach algebra. Another important example of a commutative Banach algebra is the algebra $\mathscr{A}(\mathbb{D})$ of all continuous complex-valued functions on the closed unit disc $\mathbb{D}:=\{z \in \mathbb{C}:|z| \leq 1\}$ which are analytic on the interior of $\mathbb{D}$. Here we define addition and multiplication pointwise and the norm by

$$
\|f\|=\sup \{|f(z)|: z \text { on the boundary of } \mathbb{D}\} .
$$

$\mathscr{A}(\mathbb{D})$ is called the disc algebra.

The algebra $M_{n}(\mathbb{C})$ of all $n \times n$ matrices with complex entries is a noncommutative Banach algebra with the usual linear structure and the norm defined by

$$
\|X\|=\sup \left\{\sum_{j=1}^{n}\left|x_{i j}\right|: i \in\{1, \ldots, n\}\right\},
$$


where $x_{i j}$ presents the $i j$-th entry in the matrix $X$.

If $X$ denotes a complex Banach space, then the vector space $\mathcal{L}(X)$ of all bounded linear operators on $X$ is a Banach algebra when the multiplication is defined as the composition of operators and the norm is chosen to be the usual operator norm.

The closure of a subset $B$ of an algebra $A$ will be denoted by $\bar{B}_{A}$. If the algebra under discussion is clear from the context we will only write $\bar{B}$.

By an ideal $I$ of an algebra $A$ we mean a proper two-sided ideal of $A$; i.e. $I \subsetneq A$ is a vector space which is closed under multiplication from the left and right. It is familiar that the sets $\mathcal{F}(X)$ and $\mathcal{K}(X)$ of finite-rank and compact operators on a Banach space $X$, respectively, are ideals in $\mathcal{L}(X)$.

The radical of an algebra $A$, denoted by $\operatorname{Rad}(A)$, is defined as

$$
\operatorname{Rad}(A)=\left\{a \in A: \mathbf{1}-b a \in A^{-1} \text { for all } b \in A\right\} .
$$

It is known that $\operatorname{Rad}(A)$ is a closed ideal of $A$. If $\operatorname{Rad}(A)=\{0\}$, then $A$ is said to be semisimple. Examples of semisimple Banach algebras include $M_{n}(\mathbb{C}), \mathcal{L}(X)$ and $C(K)$, where $X$ and $K$ denote, respectively, a complex Banach space and a compact Hausdorff space. Also, if $A$ is a semisimple Banach algebra, then the Banach algebra

$$
l^{\infty}(A)=\left\{\left(a_{1}, a_{2}, \ldots\right): a_{n} \in A \text { for all } n \in \mathbb{N} \text { and } \sup \left\{\left\|a_{n}\right\|: n \in \mathbb{N}\right\}<\infty\right\}
$$

equipped with norm $\left\|\left(a_{n}\right)_{n \in \mathbb{N}}\right\|=\sup \left\{\left\|a_{n}\right\|: n \in \mathbb{N}\right\}$ and componentwise addition, scalar multiplication and multiplication is again semisimple.

There are a number of ways to represent the radical of an algebra. The following representation of the radical will be useful throughout this thesis.

Theorem 1.1.3. ([25], Theorem 2.5) Let $A$ be an algebra. Then $\operatorname{Rad}(A)=\{a \in$ $\left.A: a+A^{-1} \subseteq A^{-1}\right\}$.

Let $A$ and $B$ be algebras. A linear operator $T: A \rightarrow B$ is said to be an (algebra) homomorphism if $T(a b)=T a T b$ for $a, b \in A$ and $T \mathbf{1}_{A}=\mathbf{1}_{B}$. By a "Banach algebra homomorphism" we mean a homomorphism between two Banach algebras. An isomorphism is defined as a homomorphism that is one-to-one and onto. Two algebras are said to be isomorphic if there exists an isomorphism between them.

We denote by $\mathrm{N}(T)$ the null space of a linear operator $T$. It is easy to verify that if $T$ is a non-zero linear operator which preserves multiplication, 
then $\mathrm{N}(T)$ is an ideal of $A$.

From here onwards, the reader should keep in mind the following meanings of the homomorphism $\pi$.

Remark 1.1.4. Throughout this thesis, by $\pi$ we shall mean the following:

- If $T: A \rightarrow B$ is a Banach algebra homomorphism, then $\pi$ will denote the canonical homomorphism $\pi: A \rightarrow A / \overline{\mathrm{N}(T)}$ from $A$ onto $A / \overline{\mathrm{N}(T)}$.

- If I denotes an ideal of a Banach algebra $A$, then $\pi$ is understood to be the canonical homomorphism $\pi: A \rightarrow A / \bar{I}$ from $A$ onto $A / \bar{I}$.

- In the operator case (with $X$ denoting a complex Banach space) $\pi$ will indicate the canonical homomorphism $\pi: \mathcal{L}(X) \rightarrow \mathcal{L}(X) / \mathcal{K}(X)$ from $\mathcal{L}(X)$ onto $\mathcal{L}(X) / \mathcal{K}(X)$.

For use in Section 5.2 we recall the following well-known facts.

Theorem 1.1.5. ([24], Theorem 2.6.9(b)) A linear operator with finite-dimensional domain has finite-dimensional range.

Theorem 1.1.6. ([24], Theorem 2.4.3) Every finite-dimensional subspace of a normed space is closed.

Let $\operatorname{dim} A$ denote the dimension of a vector space $A$. The following result follows from the preceding two theorems, bearing in mind that the range of a linear operator is a vector space ([24], Theorem 2.6.9(a)).

Corollary 1.1.7. If $T: A \rightarrow B$ is a Banach algebra homomorphism and $\operatorname{dim} A<$ $\infty$, then $T$ has closed range.

\subsection{Spectral theory in Banach algebras}

In this section we gather basic information on the spectral theory in Banach algebras.

Definition 1.2.1 (Spectrum). ([10], Definition, p.36) Let A be a Banach algebra. The spectrum of an element $a \in A$, denoted by $\sigma(a, A)$, is defined as follows:

$$
\sigma(a, A):=\left\{\lambda \in \mathbb{C}: \lambda \mathbf{1}-a \notin A^{-1}\right\}=\left\{\lambda \in \mathbb{C}: a-\lambda \mathbf{1} \notin A^{-1}\right\}
$$


Whenever there is no ambiguity we shall drop the $A$ in $\sigma(a, A)$. By $\sigma^{\prime}(a)$ we denote the set of all non-zero elements of $\sigma(a)$.

For $X \in M_{n}(\mathbb{C})$, we have that $\sigma(X)=\{\lambda \in \mathbb{C}: \lambda$ is an eigenvalue of $X\}$. Also, if $f \in C(K)$, where $K$ is a compact Hausdorff space, then $\sigma(f)=f(K)$.

If $a$ is an arbitrary Banach algebra element, then the set of all isolated spectral points of $a$ will be denoted by iso $\sigma(a)$, while the set of all accumulation points of the spectrum of $a$ will be denoted by acc $\sigma(a)$. The notation $\rho(a)$ will be used to denote the complement of $\sigma(a)$ and is referred to as the resolvent set of $a$.

If $T: A \rightarrow B$ is a Banach algebra homomorphism, then $\sigma(T a) \subseteq \sigma(a)$ for all $a \in A$ as $T\left(A^{-1}\right) \subseteq B^{-1}$. If the converse inclusion $\sigma(T a) \supseteq \sigma(a)$ also holds for all $a \in A$, then $T$ is said to be spectrum preserving. For a Banach algebra $A$ it is known that the canonical homomorphism $T: A \rightarrow$ $A / \operatorname{Rad}(A)$ is spectrum preserving.

For a compact set $K \subseteq \mathbb{C}$, we denote by $\eta K$ and $\partial K$, respectively, the connected hull of $K$, which is the union of $K$ with the bounded components of $\mathbb{C} \backslash K$, and the topological boundary of $K$. The spectrum of a Banach algebra element is a compact set (see Theorem 1.2.8(ii)).

Theorem 1.2.2. ([17], Theorem 5.4, p.211) Suppose that $B$ is a closed subalgebra of a Banach algebra $A$ such that $\mathbf{1}_{A} \in B \subseteq A$. If $b \in B$, then

(i) $\sigma(b, A) \subseteq \sigma(b, B)$,

(ii) $\eta \sigma(b, A)=\eta \sigma(b, B)$.

If $A$ is an algebra (with or without unit), then the quasi-product of elements $a$ and $b$ of $A$, denoted by $a \circ b$, is defined by $a \circ b:=a+b-a b$. An element $a \in A$ is said to be quasi-invertible if there exists $b \in A$ such that $a \circ b=0=b \circ a$. Denote by $q-A^{-1}$ the set of quasi-invertible elements of $A$.

In the case where $A$ is a Banach algebra without unit, the spectrum of $a \in A$, which we shall denote by $\sigma_{1}(a, A)$, is given by

$$
\sigma_{1}(a, A):=\{0\} \cup\left\{\lambda \in \mathbb{C} \backslash\{0\}: \frac{1}{\lambda} a \notin q-A^{-1}\right\}
$$

([14], p.20). By ([14], Lemma 2, p.20), $\sigma_{1}(a, A)=\sigma\left((a, 0), A_{1}\right)$ for all $a \in A$, where $A_{1}:=A \oplus \mathbb{C}$ indicates the unitization of a Banach algebra $A$.

The following result introduces an interesting relationship between the spectrum relative to a Banach algebra and the spectrum relative to the unitization of the Banach algebra. 
Proposition 1.2.3. ([14], Proposition 5, p.16) If $A$ is an algebra and $a \in A$, then $a$ is quasi-invertible if and only if $\mathbf{1}-a$ is invertible.

Hence, if $A$ is a Banach algebra, then

$$
\sigma^{\prime}(a, A)=\left\{\lambda \in \mathbb{C} \backslash\{0\}: \frac{1}{\lambda} a \notin q-A^{-1}\right\}=\sigma^{\prime}\left((a, 0), A_{\mathbf{1}}\right) .
$$

From Proposition 1.2.3 we have that $\sigma^{\prime}(a, A)=\sigma_{1}^{\prime}(a, A)$ for all elements $a$ of a Banach algebra $A$.

Proposition 1.2.4. Suppose that $B$ is a closed subalgebra of a Banach algebra A. If $b \in B$, then

(i) $\sigma\left((b, 0), A_{\mathbf{1}}\right) \subseteq \sigma\left((b, 0), B_{\mathbf{1}}\right)$,

(ii) $\eta \sigma\left((b, 0), A_{\mathbf{1}}\right)=\eta \sigma\left((b, 0), B_{\mathbf{1}}\right)$.

The above result follows from Theorem 1.2.2 applied to $A_{1}$ and $B_{1}$ where the condition $\mathbf{1}_{A_{1}}=(0,1)=\mathbf{1}_{B_{1}} \in B_{1}$ always holds.

In the following theorem, if we add the assumption $\mathbf{1}_{A} \in B$, then the result will be clear from Theorem 1.2.2(ii). Here we show that the non-zero spectrum of an element with finite spectrum relative to a Banach algebra (not necessarily unital) and the non-zero spectrum of this element relative to a "larger" Banach algebra in general coincide. This fact will come in handy when proving one of the main results in Section 5.2.

Theorem 1.2.5. Suppose that $B$ is a closed subalgebra of a Banach algebra $A$. If $b \in B$ is such that $\sigma_{1}(b, B)$ is finite, then $\sigma_{1}^{\prime}(b, B)=\sigma^{\prime}(b, A)$.

Proof. Suppose that $b \in B$. Using Propositions 1.2.3 and 1.2.4(ii) and the finiteness of $\sigma_{1}(b, B)$, we have that $\sigma_{1}^{\prime}(b, B)=\sigma^{\prime}\left((b, 0), B_{1}\right)=\sigma^{\prime}\left((b, 0), A_{\mathbf{1}}\right)=$ $\sigma^{\prime}(b, A)$.

Definition 1.2.6 (Spectral radius). ([10], Definition, p.36) Let $A$ be a Banach algebra and $a \in A$. The spectral radius $r(a, A)$ is defined as follows:

$$
r(a, A):=\sup \{|\lambda|: \lambda \in \sigma(a, A)\}
$$

It suffices to write $r(a)$ if the Banach algebra being discussed is clear from the context.

If $T: A \rightarrow B$ is a Banach algebra homomorphism, then $r(T a) \leq r(a)$ for all $a \in A$ since the inclusion $\sigma(T a) \subseteq \sigma(a)$ holds for all $a \in A$. If we also have that $r(T a) \geq r(a)$ for all $a \in A$, then $T$ is said to be spectral radius 
preserving. Obviously, if $T$ is spectrum preserving, then $T$ is spectral radius preserving. We point out the following fact for spectral radius preserving Banach algebra homomorphisms.

Lemma 1.2.7. If $T: A \rightarrow B$ is a Banach algebra homomorphism which is spectral radius preserving, then $\mathrm{N}(T) \subseteq \operatorname{Rad}(A)$.

Proof. Let $a \in \mathrm{N}(T)$. Then $T(a b)=0$ for all $b \in A$. By assumption we have that $r(a b)=r(T(a b))=0$ for all $b \in A$, and hence $a \in \operatorname{Rad}(A)$.

Theorem 1.2.8 (I.M. Gelfand). ([10], Theorem 3.2.8) Let A be a Banach algebra and $a \in A$. Then

(i) $\lambda \mapsto(\lambda \mathbf{1}-a)^{-1}$ is analytic on $\rho(a)$,

(ii) $\sigma(a)$ is compact and non-empty,

(iii) $r(a)=\lim _{n \rightarrow \infty}\left\|a^{n}\right\|^{\frac{1}{n}}$.

The function in (i) is called the resolvent of $a$. It is known that

$$
(\mu \mathbf{1}-a)^{-1}-(\lambda \mathbf{1}-a)^{-1}=(\lambda-\mu)(\lambda \mathbf{1}-a)^{-1}(\mu \mathbf{1}-a)^{-1},
$$

where $\lambda, \mu \in \rho(a)$. The equation above is referred to as the resolvent equation or resolvent identity ([17], pp.202-203).

From statement (ii) in Theorem 1.2.8 we have that the spectrum is a closed and bounded subset of $\mathbb{C}$, while a consequence of statement (iii) is that $r(a) \leq\|a\|$ for all Banach algebra elements $a$. The latter fact will be useful in Proposition 6.1.8.

Proposition 1.2.9. ([10], Corollary 3.2.10) Let $A$ be a Banach algebra and $a, b \in$ A. If $a b=b a$, then $\sigma(a+b) \subseteq \sigma(a)+\sigma(b)$ and $\sigma(a b) \subseteq \sigma(a) \sigma(b)$. Hence $r(a+b) \leq r(a)+r(b)$ and $r(a b) \leq r(a) r(b)$.

An element of a Banach algebra is said to be quasinilpotent if its spectrum consists only of the zero-element. $\operatorname{By} \mathrm{QN}(A)$ we denote the set of all quasinilpotent elements of a Banach algebra $A$. We point out that $\mathrm{QN}(A)=$ $\operatorname{Rad}(A)$ whenever $A$ is commutative ([10], Remark 1, p.71).

Definition 1.2.10 (Generalized Drazin inverse). ([22], Definition 2.3) Let $A$ be a Banach algebra. An element $a \in A$ is said to be generalized Drazin invertible if there exists an element $b \in A$ such that

$$
a b=b a, b a b=b \text { and } a-a b a \in \mathrm{QN}(A) .
$$


Such $b$, if it exists, is unique; it is called the generalized Drazin inverse of $a$ and will be denoted by $a^{D}$. If $A^{D}$ denotes the set of all generalized Drazin invertible elements of $A$, then it is known that $a \in A^{D}$ if and only if $0 \notin$ acc $\sigma(a)$. We remark that, in the literature, generalized Drazin invertible elements are often called almost invertible elements.

\subsection{Functional Calculus}

The algebra of all complex-valued functions defined and holomorphic on an open set $\Omega \subseteq \mathbb{C}$ will be denoted by $H(\Omega)$.

Proposition 1.3.1. ([10], p.43) Let $A$ be a Banach algebra and $a \in A$. If $\Omega$ is an open set containing $\sigma(a)$ and $\Gamma$ is a smooth contour included in $\Omega$ and surrounds $\sigma(a)$, then the function $f \mapsto f(a)=\frac{1}{2 \pi i} \int_{\Gamma} f(\lambda)(\lambda \mathbf{1}-a)^{-1} d \lambda$ from $H(\Omega)$ into $A$ is well-defined.

Theorem 1.3.2 (Holomorphic Functional Calculus). ([10], Theorem 3.3.3) Let $A$ be a Banach algebra and $a \in A$. Suppose that $\Omega$ is an open set containing $\sigma(a)$ and that $\Gamma$ is a smooth contour included in $\Omega$ and surrounding $\sigma(a)$. Then the function defined in Proposition 1.3.1 has the following properties:

(1) $\left(f_{1}+f_{2}\right)(a)=f_{1}(a)+f_{2}(a)$;

(2) $f_{1}(a) f_{2}(a)=\left(f_{1} f_{2}\right)(a)=f_{2}(a) f_{1}(a)$;

(3) $1(a)=\mathbf{1}$ and $I(a)=a$, where 1 and $I$ are the unit and identity functions on $\mathbb{C}$, respectively;

(4) $\sigma(f(a))=f(\sigma(a))$

for all $f_{1}, f_{2}, f \in H(\Omega)$.

Number (4) above is called the spectral mapping theorem for holomorphic functions ([17], p.208), which we will only refer to as the spectral mapping theorem.

Theorem 1.3.3. ([10], Theorem 3.3.4) Let $A$ be a Banach algebra. Suppose that $a \in A$ has a disconnected spectrum and that $U_{0}$ and $U_{1}$ are disjoint open sets satisfying

$$
\sigma(a) \subseteq U_{0} \cup U_{1}, \sigma(a) \cap U_{0} \neq \varnothing \text { and } \sigma(a) \cap U_{1} \neq \varnothing
$$


Let $f \in H\left(U_{0} \cup U_{1}\right)$ be defined by

$$
f(\lambda)= \begin{cases}0 & \text { if } \lambda \in U_{0} \\ 1 & \text { if } \lambda \in U_{1}\end{cases}
$$

Then $p:=f(a)$ is a non-trivial idempotent commuting with a satisfying $\sigma(p a)=$ $\left(\sigma(a) \cap U_{1}\right) \cup\{0\}$ and $\sigma((\mathbf{1}-p) a)=\left(\sigma(a) \cap U_{0}\right) \cup\{0\}$.

The idempotent $p$ in Theorem 1.3.3 is called the spectral idempotent of a corresponding to the set $\sigma(a) \cap U_{1}$. In the case where $\sigma(a) \cap U_{1}=\left\{\lambda_{0}\right\}$, that is $\lambda_{0} \in$ iso $\sigma(a)$, then $p$ is said to be the spectral idempotent of a corresponding to $\lambda_{0}$ and is given by

$$
p\left(a, \lambda_{0}\right):=\frac{1}{2 \pi i} \int_{\Gamma}(\lambda \mathbf{1}-a)^{-1} d \lambda
$$

where $\Gamma$ is a circle centred at $\lambda_{0}$, separating $\lambda_{0}$ from the remaining spectrum of $a$. Then, $p\left(a, \lambda_{0}\right)=0$ if and only if $\lambda_{0} \notin \sigma(a)$.

The following fact will be required in Lemma 2.2.7 and Theorem 4.3.2.

Lemma 1.3.4. Let $A$ be a Banach algebra and $a \in A$. Then $p(\lambda \mathbf{1}-a, 0)=p(a, \lambda)$ for all $\lambda \in$ iso $\sigma(a)$.

Proof. Let $\lambda \in$ iso $\sigma(a)$. Then $0 \in$ iso $\sigma(\lambda \mathbf{1}-a)$, and we choose $\Gamma$ to be a circle centred at 0 , separating 0 from the rest of $\sigma(\lambda \mathbf{1}-a)$. Consequently,

$$
p(\lambda \mathbf{1}-a, 0)=\frac{1}{2 \pi i} \int_{\Gamma}(\mu-(\lambda \mathbf{1}-a))^{-1} d \mu=\frac{1}{2 \pi i} \int_{\Gamma}(a-(\lambda-\mu) \mathbf{1})^{-1} d \mu .
$$

Let $z=\lambda-\mu$. Then $d z=-d \mu$ and $\Gamma^{*}=\lambda+\Gamma$ is a circle centred at $\lambda$, separating $\lambda$ from the rest of $\sigma(a)$. Consequently, the identities

$$
p(\lambda \mathbf{1}-a, 0)=\frac{1}{2 \pi i} \int_{\Gamma^{*}}(a-z \mathbf{1})^{-1}(-d z)=\frac{1}{2 \pi i} \int_{\Gamma^{*}}(z \mathbf{1}-a)^{-1} d z=p(a, \lambda)
$$

hold.

\subsection{Ideals in Banach algebras}

In this section we state a few useful results pertaining to ideals in Banach algebras. To begin with, we recall the fact that, if $I$ is an ideal of a Banach algebra $A$, then $I$ and $\bar{I}$ have the same set of idempotents ([10], p.107). 
Definition 1.4.1 (Riesz element). ([12], p.53) Let I be a closed ideal of a Banach algebra $A$. An element $a \in A$ is said to be Riesz w.r.t. I if $\sigma(a+I)=\{0\}$.

Definition 1.4.2 (Riesz point of spectrum). (Aupetit (1986), see [30], p.150) Let $I$ be an ideal of a Banach algebra $A$ and $a \in A$. Then $\lambda \in$ iso $\sigma(a)$ is called $a$ Riesz point of $\sigma(a)$ relative to $I$ if $p(a, \lambda) \in I$.

Definition 1.4.3 (Inessential ideal). ([10], p.106) An ideal I of a Banach algebra $A$ is said to be inessential if the spectrum of each element in $I$ is either finite or a sequence converging to zero.

It is known that $\mathcal{F}(X)$ and $\mathcal{K}(X)$ are examples of inessential ideals in $\mathcal{L}(X)$. The fact that $\bar{I}$ is an inessential ideal of a Banach algebra $A$ whenever $I$ is an inessential ideal of $A$ ([10], Corollary 5.7.6) will often be used without any mention.

Definition 1.4.4 (Riesz property of a homomorphism). ([20], p.432) A Banach algebra homomorphism $T: A \rightarrow B$ is said to have the Riesz property if $\mathrm{N}(T)$ is an inessential ideal.

If $a$ denotes a Banach algebra element, then a point $z \in \sigma(a)$ is called a pole of order $k$ of $(\lambda \mathbf{1}-a)^{-1}$, where $\lambda \notin \sigma(a)$, if $z \in$ iso $\sigma(a)$ and $k$ is the smallest natural number such that $(z \mathbf{1}-a)^{k} p(a, z)=0$.

The following result tells us when a Riesz point of the spectrum will also be a pole and will be useful in the sequel.

Lemma 1.4.5. ([30], Lemma 2.1) Let $A$ be a semisimple Banach algebra, I be an inesssential ideal of $A$ and $a \in A$. Then $\alpha$ is a Riesz point of $\sigma(a)$ relative to $I$ if and only if $\alpha$ is a pole of $(\lambda \mathbf{1}-a)^{-1}$ and $p(a, \alpha) \in I$.

For an inessential ideal $I$ of a Banach algebra $A$ and $a \in A$, we denote the set $\sigma(a) \backslash\{\lambda \in \sigma(a): \lambda$ is a Riesz point of $\sigma(a)$ relative to $I\}$ by $D(a, I)$. We remark that Corollary 2.1.4 (a result that will often be referred to in the sequel of this thesis) relies on the following theorem.

Theorem 1.4.6 (Perturbation by inessential elements). ([10], Theorem 5.7.4) Let $A$ be a Banach algebra and $I$ be an inessential ideal of $A$. Then $\sigma(a+\bar{I}) \subseteq$ $D(a, I)$ and $\eta D(a, I)=\eta \sigma(a+\bar{I})$ for all $a \in A$. 


\subsection{Fredholm theory in Banach algebras}

The basic concepts and results from Fredholm theory in Banach algebras needed for our study will be reviewed briefly in this section.

Definition 1.5.1 (Fredholm, Weyl and Browder elements). ([20], pp.431-432) Let $T: A \rightarrow B$ be a Banach algebra homomorphism. An element $a \in A$ is called

- Fredholm if $T a \in B^{-1}$,

- Weyl if there exist elements $b \in A^{-1}$ and $c \in \mathrm{N}(T)$ such that $a=b+c$,

- Browder if there exist commuting elements $b \in A^{-1}$ and $c \in \mathrm{N}(T)$ such that $a=b+c$,

- almost invertible Fredholm if it is Fredholm and generalized Drazin invertible.

Denote by $\mathcal{F}_{T}, \mathcal{W}_{T}, \mathcal{B}_{T}$ and $A^{D} \cap \mathcal{F}_{T}$ the sets of Fredholm, Weyl, Browder and almost invertible Fredholm elements of $A$ (relative to $T$ ), respectively.

In the following example we point out additional representations for the Weyl operators on a Banach space for ease of reference in the sequel.

Example 1.5.2. ([20], p.431; [13], Corollary 2.8) Let $X$ be a Banach space and $\pi: \mathcal{L}(X) \rightarrow \mathcal{L}(X) / \mathcal{K}(X)$ be the canonical homomorphism. Then:

(a) $T \in \mathcal{F}_{\pi}$ if and only if $\operatorname{dim} \mathrm{N}(T)<\infty, T(X)$ is closed and $\operatorname{dim}(X / T(X))<\infty$.

(b) $T \in \mathcal{B}_{\pi}$ if and only if $T \in \mathcal{F}_{\pi}$ with finite ascent and descent.

(c) $T \in \mathcal{W}_{\pi}$ if and only if $T \in \mathcal{F}_{\pi}$ with index zero if and only if $T \in(\mathcal{L}(X))^{-1}+$ $\mathcal{K}(X)$ if and only if $T \in(\mathcal{L}(X))^{-1}+\mathcal{F}(X)$.

Example 1.5.3. ([20], p.432) Let $K_{1}$ and $K_{2}$ be compact Hausdorff spaces and $A:=C\left(K_{1}\right)$ and $B:=C\left(K_{2}\right)$ be the Banach algebras of continuous complexvalued functions on $K_{1}$ and $K_{2}$, respectively. Consider the homomorphism $T$ : $A \rightarrow B$ defined by $T f=f \circ \theta$, where $\theta: K_{2} \rightarrow K_{1}$ is a continuous map. Then (a) $f \in \mathcal{F}_{T}$ if and only if $\theta\left(K_{2}\right) \cap \mathrm{N}(f)=\varnothing$,

(b) $f \in \mathcal{W}_{T}$ if and only if its restriction to $\theta\left(K_{2}\right)$ has an invertible extension to $K_{1}$.

In the previous example, since $A$ is a commutative Banach algebra, the sets of Browder and Weyl elements coincide. 
Proposition 1.5.4. ([20], (1.4) and Theorem 1; [29], Corollary 2.5) Let $T: A \rightarrow B$ be a Banach algebra homomorphism. Then

$$
A^{-1} \subseteq A^{D} \cap \mathcal{F}_{T} \subseteq \mathcal{B}_{T} \subseteq \mathcal{W}_{T} \subseteq \mathcal{F}_{T}
$$

Moreover, $A^{D} \cap \mathcal{F}_{T}=\mathcal{B}_{T}$ if and only if $T$ has the Riesz property.

By using the theory of generalized Drazin invertible elements we provide an alternative (simpler) proof of a result that follows from ([18], Proposition 2.1). This result, assuming boundedness of the homomorphism, was mentioned by R. Harte in [20], and in [29] H. Mouton and H. Raubenheimer observed that it was true for unbounded homomorphisms as well, upon which they used it to extend a theorem of Harte in [20].

We recall the identity $p(a, 0)=\mathbf{1}-a^{D} a$ for a generalized Drazin invertible Banach algebra element $a$ ([22], Theorem 5.4(iv)).

Proposition 1.5.5. Let $T: A \rightarrow B$ be a Banach algebra homomorphism. If a $\in$ $A^{D} \cap \mathcal{F}_{T}$, then $p \in \mathrm{N}(T)$, where $p$ is the spectral idempotent of a corresponding to 0 .

Proof. The inclusion $T\left(A^{D}\right) \subseteq B^{D}$ follows easily from the fact that $T$ is a homomorphism. In particular one has that $(T a)^{D}=T a^{D}$ for all $a \in A^{D}$.

Consequently, if $a \in A^{D} \cap \mathcal{F}_{T}$, then

$$
T p=T\left(\mathbf{1}-a^{D} a\right)=\mathbf{1}-T a^{D} T a=\mathbf{1}-(T a)^{D} T a=\mathbf{1}-(T a)^{-1} \mathrm{~T} a=0,
$$

that is $p \in \mathrm{N}(T)$.

Let $T: A \rightarrow B$ be a Banach algebra homomorphism. Using the sets $\mathcal{F}_{T}$, $\mathcal{W}_{T}$ and $\mathcal{B}_{T}$ we recall the following spectra introduced by Harte in ([20], p.433-434). Let $a \in A$.

- The Fredholm spectrum of $a$ is given by

$$
\sigma(T a)=\left\{\lambda \in \mathbb{C}: \lambda \mathbf{1}-a \notin \mathcal{F}_{T}\right\} .
$$

- The Weyl spectrum of $a$ is given by

$$
\omega_{T}(a)=\left\{\lambda \in \mathbb{C}: \lambda \mathbf{1}-a \notin \mathcal{W}_{T}\right\}=\bigcap_{c \in \mathrm{N}(T)} \sigma(a+c) .
$$


- The Browder spectrum of $a$ is given by

$$
\beta_{T}(a)=\left\{\lambda \in \mathbb{C}: \lambda \mathbf{1}-a \notin \mathcal{B}_{T}\right\}=\bigcap_{\substack{c \in \mathrm{N}(T) \\ a c=c a}} \sigma(a+c) .
$$

- The almost invertible Fredholm spectrum of $a$ is given by

$$
\left\{\lambda \in \mathbb{C}: \lambda \mathbf{1}-a \notin A^{D} \cap \mathcal{F}_{T}\right\}=\sigma(T a) \cup \operatorname{acc} \sigma(a) .
$$

From Proposition 1.5.4 we have that

$$
\sigma(T a) \subseteq \omega_{T}(a) \subseteq \beta_{T}(a) \subseteq \sigma(T a) \cup \operatorname{acc} \sigma(a) \subseteq \sigma(a)
$$

and that $\beta_{T}(a)=\sigma(T a) \cup \operatorname{acc} \sigma(a)$ if and only if $T$ has the Riesz property. It is known that these spectra are non-empty and compact.

Theorem 1.5.7. ([28], Corollary 5.6) Let $T: A \rightarrow B$ be a Banach algebra homomorphism which satisfies the Riesz property. Then $\beta_{T}(a)=\beta_{\pi}(a)$ and $\omega_{T}(a)=$ $\omega_{\pi}(a)$ for all $a \in A$.

In the subsequent chapters, quite often, the following result will be used.

Theorem 1.5.8. ([28], Corollary 7.6, 7.8) Let $T: A \rightarrow B$ be a Banach algebra homomorphism with closed range satisfying the Riesz property. Then $\eta \sigma(T a)=$ $\eta \omega_{T}(a)=\eta \beta_{T}(a)$ for all $a \in A$.

\subsection{Banach lattice theory}

In this section, we review a few results from the theory of Banach lattices and discuss some properties of operators between them. We give here a presentation of the structural properties of Banach lattices and point out that concepts (related to convergence) can be found in [7].

Definition 1.6.1 (Vector lattice). ([7], p.2) A vector lattice (Riesz space) is a real vector space $V$ with a partial order in which, for every $x, y \in V$, the following conditions hold:

- the supremum and infimum of $x$ and $y$ both exist in $V$,

- if $x \leq y$, then $x+z \leq y+z$ for all $z \in V$,

- if $x \leq y$ and $c \in \mathbb{R}^{+}$, then $c x \leq c y$. 
For an element $x$ in a vector lattice $V$, its positive part, its negative part and its modulus are defined by

$$
x^{+}=\sup \{x, 0\}, x^{-}=\sup \{-x, 0\} \text { and }|x|=\sup \{x,-x\},
$$

respectively. Consequently, the identities $x=x^{+}-x^{-}$and $|x|=x^{+}+x^{-}$ hold. By $x_{\alpha} \downarrow x\left(x_{\alpha} \uparrow x\right)$ we mean that $\left(x_{\alpha}\right)$ is a decreasing (an increasing) net satisfying $\inf \left\{x_{\alpha}\right\}=x\left(\sup \left\{x_{\alpha}\right\}=x\right)$. We use the notations $V_{+}:=\{x \in$ $V: x=|x|\}$ to denote the positive cone in $V$ and $V_{n}^{\sim}$ to denote the order continuous dual of $V$. An operator $T$ on $V$ is said to be positive if $T V_{+} \subseteq V_{+}$.

By a Dedekind complete vector lattice $V$ we mean a vector lattice in which every non-empty subset of $V$ that is (order) bounded from above has a supremum.

If $V_{1}$ and $V_{2}$ denote vector lattices, then $T: V_{1} \rightarrow V_{2}$ is said to be an order continuous operator if $\left(T x_{\alpha}\right)$ is order convergent (see [7]) to 0 in $V_{2}$ whenever $\left(x_{\alpha}\right)$ is order convergent to 0 in $V_{1}$. It is useful to note that a positive operator $T$ is order continuous if and only if $x_{\alpha} \downarrow 0$ implies $T x_{\alpha} \downarrow 0$.

Definition 1.6.2 (Normed vector lattice). ([7], p.174) A normed vector lattice is a normed space $V$ which is also a vector lattice in which $|x| \leq|y| \Rightarrow\|x\| \leq\|y\|$ for all $x, y \in V$.

A normed vector lattice which is also a Banach space is said to be a $B a-$ nach lattice.

Definition 1.6.3 (Order continuous norm). ([7], Definition 12.7) A norm in a normed vector lattice is said to be order continuous if $x_{\alpha} \downarrow 0$ implies $\left\|x_{\alpha}\right\| \downarrow 0$.

If $V$ denotes a vector lattice, then a vector subspace $B$ of $V$ is called an order ideal if $|x| \leq|y|, x \in V$ and $y \in B$ imply $x \in B$.

Definition 1.6.4 (Band). ([35], Definition 2.8, p.61) A band in a vector lattice $V$ is an order ideal $B$ with the property that, if $D \subseteq B$ and $x$ is the supremum of $D$, then $x \in B$.

A band $B$ in a vector lattice $V$ is said to be a projection band if $V=B \oplus B^{d}$, where $B^{d}:=\{x \in V: \inf \{|x|,|y|\}=0$ for all $y \in B\}$ denotes the disjoint complement of $B$ in $V$.

Let $X$ be a Banach space and $T \in \mathcal{L}(X)$. Recall that a vector subspace $V$ of $X$ is said to be $T$-invariant if $T V \subseteq V$. 
Definition 1.6.5 (Band irreducible). ([2], Definition 9.2) Let E be a Banach lattice and $T \in \mathcal{L}(E)$. Then $T$ is said to be band irreducible on $E$ if $E$ contains no non-trivial T-invariant bands.

Definition 1.6.6. ([2], Definition 3.1 and Exercise 7, p.101; [7], Definition 14.10) We say that a Banach lattice $E$ is:

(i) an $A M$-space if $\|\sup \{x, y\}\|=\sup \{\|x\|,\|y\|\}$ for every $x, y \in E_{+}$,

(ii) an AL-space if $\|x+y\|=\|x\|+\|y\|$ for every $x, y \in E_{+}$,

(iii) a KB-space if every increasing norm bounded sequence of elements in $E_{+}$converges in norm in $E$.

A Banach lattice $E$ is said to be an $A M$-space with unit if $E$ is an $A M$-space and there exists an element $e$, called the unit, such that for each $x \in E$ we can find some $\lambda>0$ satisfying $|x| \leq \lambda$ e.

We have the following implication-scheme that will frequently be referred to (see ([11], Theorem 2.83) and ([7], Theorem 12.9 and p.225)):

$E$ is an $A L$-space $\Rightarrow E$ is a $K B$-space $\Rightarrow E$ has order continuous norm

$$
\Rightarrow E \text { is Dedekind complete }
$$

We fix the following notation that will be needed in the sequel. An operator on a Banach lattice $E$ is called regular if it can be written as a linear combination (over $\mathbb{R}$ ) of positive operators. The space of all regular operators on $E$ will be denoted by $\mathcal{L}^{r}(E)$ and, for $T \in \mathcal{L}^{r}(E)$, the regular norm (which we will abbreviate as $r$-norm) is defined by

$$
\|T\|_{r}:=\inf \{\| S||: S \in \mathcal{L}(E),|T x| \leq S|x| \text { for all } x \in E\} .
$$

In addition, $T \in \mathcal{L}^{r}(E)$ is called $r$-compact if it can be approximated in the $r$-norm by finite-rank operators. The space of all $r$-compact operators will be denoted by $\mathcal{K}^{r}(E)$.

The following result will be useful in Example 3.2.8.

Theorem 1.6.8. ([1], Theorem 5.2, Corollary, p.25) Let $X$ and $Y$ be Banach lattices, where $X$ is not a KB-space. Then $Y$ is an AM-space if and only if each compact operator $T: X \rightarrow Y$ is regular.

By a complex Banach lattice $E$ we mean the complexification $E_{\mathbb{R}}+i E_{\mathbb{R}}$ of a real Banach lattice $E_{\mathbb{R}}$. Additionally, a complex $A M$-space (respectively, $A M$-space with unit, $A L$-space and $K B$-space) is the complexification of 
a (real) $A M$-space (respectively, $A M$-space with unit, $A L$-space and $K B$ space).

If $T \in \mathcal{L}(E):=\mathcal{L}\left(E_{\mathbb{R}}\right)+i \mathcal{L}\left(E_{\mathbb{R}}\right)$, then there exist $T_{1}, T_{2} \in \mathcal{L}\left(E_{\mathbb{R}}\right)$ such that $T=T_{1}+i T_{2}$. The operator $T+i 0$, where $T \in \mathcal{L}\left(E_{\mathbb{R}}\right)$, will be denoted by $T_{\mathbb{C}}$ and we say that $T \in \mathcal{L}(E)$ is positive if $T=S_{\mathbb{C}}$ for some positive operator $S \in \mathcal{L}\left(E_{\mathbb{R}}\right)$.

In this thesis we shall mainly be concerned with complex Banach lattices and use the term "Banach lattice" to mean "complex Banach lattice". However, in the sequel, whenever needed, we shall denote a real Banach lattice by $E_{\mathbb{R}}$.

If $E$ is a Banach lattice, then $\mathcal{L}^{r}(E):=\mathcal{L}^{r}\left(E_{\mathbb{R}}\right)+i \mathcal{L}^{r}\left(E_{\mathbb{R}}\right)$ is a Banach algebra when equipped with the $r$-norm ([2], Corollary 3.27). In addition, if $E$ is Dedekind complete, then $\mathcal{L}^{r}(E)$ is a Dedekind complete Banach lattice ([2], Theorem 1.32).

In view of Remark 1.1.4 we point out here that $\pi_{r}$ means the canonical homomorphism $\pi_{r}: \mathcal{L}^{r}(E) \rightarrow \mathcal{L}^{r}(E) / \mathcal{K}^{r}(E)$.

It is well-known that $\overline{\mathcal{F}(E)}_{\mathcal{L}(E)} \neq \mathcal{K}(E)$ in general. It turns out that if $E$ is either $A L$ or $A M$, then the two sets coincide. The following observation will be used when proving Corollary 5.5.9.

Lemma 1.6.9. If a Banach lattice $E$ is either $A L$ or $A M$, then $\mathcal{K}^{r}(E)=\mathcal{K}(E)$.

Proof. For an $A M$-space $E$ the identity was established in ([5], Lemma 7.6(b)).

Suppose that $E$ is an $A L$-space. Using the fact that every $A L$-space is a $K B$-space, the identity $\mathcal{L}(E)=\mathcal{L}^{r}(E)$ holds by ([2], Theorem 3.9), and hence

$$
\mathcal{K}^{r}(E)=\overline{\mathcal{F}(E)}_{\mathcal{L}^{r}(E)}=\overline{\mathcal{F}(E)}_{\mathcal{L}(E)} .
$$

In view of ([35], Theorem 2.4, p.239) and ([2], Theorem 4.12) the result then follows.

\subsection{Ordered Banach algebras}

In this section we give the general background on ordered Banach algebras needed for our study.

Definition 1.7.1 (Algebra cone). ([34], p.492) A non-empty subset $C$ of a Banach algebra $A$ is said to be an algebra cone of $A$ if $C$ satisfies the following properties: 
- $C+C \subseteq C$,

- $\lambda C \subseteq C$ for all $\lambda \in \mathbb{R}^{+}$,

- $C \cdot C \subseteq C$,

- $\mathbf{1} \in C$.

Let $C$ be an algebra cone of a Banach algebra $A$. Then $C$ is said to be generating if $A=$ span $C$, that is, the elements of $A$ are linear combinations of elements of $C$, and proper whenever $C \cap-C=\{0\}$. If there exists a constant $\beta>0$ such that $0 \leq a \leq b$ implies that $\|a\|<\beta\|b\|$, then $C$ is said to be normal. It is known that every normal algebra cone is proper.

$C$ induces an ordering " $\leq$ " on $A$ in the following way: If $a, b \in A$, then

$$
a \leq b \text { if and only if } b-a \in C \text {. }
$$

A Banach algebra $A$ containing an algebra cone, say, $C$ is called an ordered Banach algebra $(O B A)$, which we shall denote by $(A, C)$.

Considering the ordering that $C$ induces we find that $C=\{a \in A: a \geq$ $0\}$ and therefore the elements of $C$ are called positive. When writing $a>0$, we mean $a \geq 0$ and $a \neq 0$. If $a \in-C$, that is, $a \leq 0$, then $a$ is said to be negative.

We now supply examples of some of the more well-known OBAs.

- $\left(\mathbb{C}, \mathbb{R}^{+}\right)$is an OBA.

- If $K$ is a compact Hausdorff space, then $(C(K), C)$ is an OBA, where $C=\left\{f \in C(K): f(x) \in \mathbb{R}^{+}\right.$for all $\left.x \in K\right\}$.

- If $C=\left\{f \in \mathscr{A}(\mathbb{D}): f(z) \in \mathbb{R}^{+}\right.$for all $\left.z \in \mathbb{D}\right\}$, then the disc algebra $(\mathscr{A}(\mathbb{D}), C)$ is an OBA.

- Let $C:=M_{n}\left(\mathbb{R}^{+}\right)$and $C^{\prime}$ be the subset of $M_{n}(\mathbb{C})$ consisting of all diagonal matrices with non-negative real entries. Then $\left(M_{n}(\mathbb{C}), C\right)$ and $\left(M_{n}(\mathbb{C}), C^{\prime}\right)$ are OBAs. If $M_{n}^{u}(\mathbb{C})$ and $M_{n}^{l}(\mathbb{C})$ denote, respectively, the algebras of upper triangular matrices and lower triangular matrices in $M_{n}(\mathbb{C})$, then $\left(M_{n}^{u}(\mathbb{C}), C \cap M_{n}^{u}(\mathbb{C})\right)$ and $\left(M_{n}^{u}(\mathbb{C}), C^{\prime} \cap M_{n}^{u}(\mathbb{C})\right)$ are OBAs. Also, $\left(M_{n}^{l}(\mathbb{C}), C \cap M_{n}^{l}(\mathbb{C})\right)$ and $\left(M_{n}^{l}(\mathbb{C}), C^{\prime} \cap M_{n}^{l}(\mathbb{C})\right)$ are OBAs. 
In the sequel we indicate $C \cap M_{n}^{u}(\mathbb{C})$ and $C \cap M_{n}^{l}(\mathbb{C})$ by $M_{n}^{u}\left(\mathbb{R}^{+}\right)$ and $M_{n}^{l}\left(\mathbb{R}^{+}\right)$, respectively.

- If $E$ denotes a Banach lattice, then $(\mathcal{L}(E), K)$ and $\left(\mathcal{L}^{r}(E), K\right)$ are OBAs, where $K:=\left\{T \in \mathcal{L}(E): T E_{+} \subseteq E_{+}\right\}$denotes the cone of positive operators on $E$.

We mention that the algebra cones given in the examples above are closed and normal. The following examples illustrate how new OBAs can be constructed from existing OBAs.

If $(A, C)$ is an OBA and $l^{\infty}(C):=\left\{\left(c_{1}, c_{2}, \ldots\right) \in l^{\infty}(A): c_{n} \in C\right.$ for all $n \in$ $\mathbb{N}\}$, then a direct verification shows that $\left(l^{\infty}(A), l^{\infty}(C)\right)$ is an OBA. Furthermore, if $C$ is closed (normal), then $l^{\infty}(C)$ is closed (normal).

Let $n \in \mathbb{N}$ and suppose that $\left(A_{i}, C_{i}\right)$ is an OBA for each $i \in\{1,2, \ldots, n\}$. If we define the direct sum of a finite number of algebras in the usual way, then $A:=\bigoplus_{i=1}^{n} A_{i}$ is another example of an OBA with algebra cone $C:=$ $\bigoplus_{i=1}^{n} C_{i}$. We mention that, if $C_{i}$ is closed (normal) for all $i \in\{1,2, \ldots, n\}$, then $C$ is closed (normal).

Definition 1.7.2. Let $(A, C)$ be an $O B A$. Then $C$ is said to be inverse-closed if for every invertible element $a \in C$ we have that $a^{-1} \in C$.

A simple example of an inverse-closed algebra cone is $\mathbb{R}^{+}$in $\mathbb{C}$. Also, if $C$ is an inverse-closed algebra cone of a Banach algebra $A$, then $l^{\infty}(C)$ is an inverse-closed algebra cone of $l^{\infty}(A)$. More examples of inverse-closed algebra cones include $C$ in $C(K)$ and $C^{\prime}$ in $M_{n}(\mathbb{C})$. (See examples on previous page).

If $n \in \mathbb{N}$ and $\left(A_{i}, C_{i}\right)$ is an OBA for each $i \in\{1,2, \ldots, n\}$, then it is an easy exercise to show that $C:=\bigoplus_{i=1}^{n} C_{i}$ is an inverse-closed algebra cone in $A:=\bigoplus_{i=1}^{n} A_{i}$ whenever $C_{i}$ is an inverse-closed algebra cone in $A_{i}$ for all $i \in\{1,2, \ldots, n\}$. In addition, if at least one $C_{i}$ is not inverse-closed, then $C$ will not be inverse-closed in $A$.

\subsection{Spectral theory in OBAs}

In this section we highlight a number of important results on the spectral theory of positive elements in OBAs. The reader is reminded of Remark 1.1.4. 
Theorem 1.8.1. ([32], Theorem 3.2) Let $(A, C)$ be an OBA with closed algebra cone $C$ and suppose that $a \in C$. If $r(a)$ is a pole of order $k$ of $(\lambda \mathbf{1}-a)^{-1}$, so that $(\lambda \mathbf{1}-a)^{-1}=\sum_{j=-k}^{\infty}(\lambda-r(a))^{j} a_{j}$, then there exists $0 \neq u:=a_{-k} \in C$ such that $a u=u a=r(a) u$.

Let $(A, C)$ be an OBA and $a, b \in A$. If $0 \leq a \leq b$ (relative to $C$ ) implies that $r(a) \leq r(b)$, then we say that the spectral radius function in $(A, C)$ is monotone. If $I$ is an ideal of $A$, then we say that the spectral radius in $(A / \bar{I}, \pi C)$ is weakly monotone, if $0 \leq a \leq b$ (relative to $C$ ) implies that $r(\pi a) \leq r(\pi b)$.

It is well-known that, if $C$ is normal, then the spectral radius function in $(A, C)$ is monotone (see [34], Theorem 4.1(1)).

The following theorem, due to H. Raubenheimer and S. Rode [34], will be of fundamental importance when proving several results for positive elements in OBAs.

Theorem 1.8.2. ([34], Theorem 5.2) Let $(A, C)$ be an OBA with closed algebra cone $C$ such that the spectral radius function in $(A, C)$ is monotone. If $a \in C$, then $r(a) \in \sigma(a)$.

The following result will be useful in proving Theorem 5.5.4.

Theorem 1.8.3. ([34], Theorem 5.3) Let $(A, C)$ be an OBA with closed algebra cone $C$ and let $I$ be a closed ideal of $A$ such that the spectral radius function in $(A / I, \pi C)$ is weakly monotone. If $a \in C$, then $r(\pi a) \in \sigma(\pi a)$.

For use in Lemma 6.2.2 we state the following result.

Theorem 1.8.4. ([34], Theorem 6.2) Let $(A, C)$ be an $O B A$ and let I be a closed ideal in $A$ such that the spectral radius function in $(A / I, \pi C)$ is weakly monotone. If $a, b \in A$ satisfy $0 \leq a \leq b$ and $b$ is Riesz w.r.t. I, then $a$ is Riesz w.r.t. I.

The following result will be used to prove Proposition 1.9.22.

Theorem 1.8.5. ([32], Theorem 4.3) Let $(A, C)$ be an OBA with closed algebra cone $C$ such that the spectral radius function in $(A, C)$ is monotone and let $I$ be a closed inessential ideal such that spectral radius function in $(A / I, \pi C)$ is weakly monotone. Also suppose that $a, b \in A$ satisfy $0 \leq a \leq b$ and $r(a)=r(b)$. If $r(b)$ is a Riesz point of $\sigma(b)$ relative to $I$, then $r(a)$ is a Riesz point of $\sigma(a)$ relative to $I$.

The following result will be required in Lemma 6.2.2 . 
Proposition 1.8.6. ([31], Proposition 4.6) Let $(A, C)$ be an OBA with closed algebra cone $C$ and $a \in C$. If $\lambda>r(a)$, then $(\lambda \mathbf{1}-a)^{-1} \in C$.

Proposition 1.8.7. ([15], Proposition 4.2) Let $(A, C)$ be an OBA with closed and inverse-closed algebra cone $C$. If $a \in C$, then $0 \leq a \leq r(a) \mathbf{1}$.

The following corollary is an immediate consequence of Proposition 1.8.7 and will be used to prove Lemma 2.2.9.

Corollary 1.8.8. Let $(A, C)$ be an $O B A$ with proper, closed and inverse-closed algebra cone $C$. If $a \in C \cap \mathrm{QN}(A)$, then $a=0$.

Proof. Let $a \in C \cap \mathrm{QN}(A)$. From Proposition 1.8.7 we have that $0 \leq a \leq$ $r(a) \mathbf{1}=0$, and hence $a \in C \cap-C$. The result follows from the fact that $C$ is proper.

\subsection{Irreducibility in OBAs}

The theory of irreducibility in an OBA was developed by Alekhno in [6]. In this section we record some concepts and important results from [6] which will serve as the background needed to study spectral properties of elements in OBAs which have disjunctive products.

We start our discussion by defining a few concepts (known from the theory of vector lattices) in the context of OBAs.

Definition 1.9.1 (Infimum (Supremum)). Let $(A, C)$ be an $O B A$ and $B \subseteq A$. An element $x \in A$ is said to be the infimum (supremum) of $B$ if $x \leq b(b \leq x)$ for all $b \in B$, and, if $y \in A$ is such that $y \leq b(b \leq y)$ for all $b \in B$, then $y \leq x$ $(x \leq y)$.

We write $x=\inf B(x=\sup B)$.

Definition 1.9.2. Let $(A, C)$ be an $O B A$ and $\left(a_{\alpha}\right)$ a net in $A$. We write $a_{\alpha} \uparrow a$ $\left(a_{\alpha} \downarrow a\right)$ if

- $\left(a_{\alpha}\right)$ is increasing (decreasing),

- $\sup \left\{a_{\alpha}\right\}\left(\inf \left\{a_{\alpha}\right\}\right)$ exists with $\sup \left\{a_{\alpha}\right\}=a\left(\inf \left\{a_{\alpha}\right\}=a\right)$.

A subset $B$ of an OBA $(A, C)$ is said to be order-bounded above whenever there exists $a \in A$ satisfying $b \leq a$ for all $b \in B$. 
Definition 1.9.3 (Dedekind complete). An OBA $(A, C)$ is said to be Dedekind complete if every non-empty order-bounded above set in $A$ has a supremum.

Some examples of Dedekind complete OBAs include $\left(\mathbb{C}, \mathbb{R}^{+}\right.$) (or more generally $\left(\mathbb{C}^{n},\left(\mathbb{R}^{n}\right)^{+}\right),\left(l^{\infty}(\mathbb{C}), l^{\infty}\left(\mathbb{R}^{+}\right)\right)$and $\left(M_{n}(\mathbb{C}), M_{n}\left(\mathbb{R}^{+}\right)\right)$. We mention that the main tool here is the fact that $\mathbb{R}$ satisfies the completeness axiom. As an illustration we present a proof which shows that $M_{2}(\mathbb{C})$ is a Dedekind complete OBA.

Example 1.9.4. The $O B A\left(M_{2}(\mathbb{C}), M_{2}\left(\mathbb{R}^{+}\right)\right)$is Dedekind complete.

Suppose that $B \subseteq M_{2}(\mathbb{C})$ is order-bounded above; that is, there exist elements $x_{1}, x_{2}, x_{3}, x_{4} \in \mathbb{C}$ such that $\left(\begin{array}{ll}a & b \\ c & d\end{array}\right) \leq\left(\begin{array}{ll}x_{1} & x_{2} \\ x_{3} & x_{4}\end{array}\right)$ for all $\left(\begin{array}{ll}a & b \\ c & d\end{array}\right) \in B$ $(a, b, c, d \in \mathbb{C})$. Hence

$\left(\begin{array}{ll}\operatorname{Re} x_{1}-\operatorname{Re} a+i\left(\operatorname{Im} x_{1}-\operatorname{Im} a\right) & \operatorname{Re} x_{2}-\operatorname{Re} b+i\left(\operatorname{Im} x_{2}-\operatorname{Im} b\right) \\ \operatorname{Re} x_{3}-\operatorname{Re} c+i\left(\operatorname{Im} x_{3}-\operatorname{Im} c\right) & \operatorname{Re} x_{4}-\operatorname{Re} d+i\left(\operatorname{Im} x_{4}-\operatorname{Im} d\right)\end{array}\right) \in M_{2}\left(\mathbb{R}^{+}\right)$,

for all $a, b, c, d \in \mathbb{C}$ such that $\left(\begin{array}{ll}a & b \\ c & d\end{array}\right) \in B$.

Let $a_{1}:=\sup \left\{\operatorname{Re} a:\left(\begin{array}{ll}a & b \\ c & d\end{array}\right) \in B\right.$ for some $\left.b, c, d \in \mathbb{C}\right\}, b_{1}:=\sup \{\operatorname{Re} b$ : $\left(\begin{array}{ll}a & b \\ c & d\end{array}\right) \in B$ for some $\left.a, c, d \in \mathbb{C}\right\}, c_{1}:=\sup \left\{\operatorname{Re} c:\left(\begin{array}{ll}a & b \\ c & d\end{array}\right) \in B\right.$ for some $a, b, d \in$ $\mathbb{C}\}$ and $d_{1}:=\sup \left\{\operatorname{Re} d:\left(\begin{array}{ll}a & b \\ c & d\end{array}\right) \in B\right.$ for some $\left.a, b, c \in \mathbb{C}\right\}$ by using the completeness axiom in $\mathbb{R}$. One can easily verify that

$$
\sup B=\left(\begin{array}{cc}
a_{1}+i \operatorname{Im} x_{1} & b_{1}+i \operatorname{Im} x_{2} \\
c_{1}+i \operatorname{Im} x_{3} & d_{1}+i \operatorname{Im} x_{4}
\end{array}\right)
$$

Also, if $E$ is a Dedekind complete Banach lattice, then $\mathcal{L}^{r}(E)$ is an example of a Dedekind complete OBA. If, in addition, $E$ is either $A L$ or $A M$ with unit, then $\mathcal{L}(E)=\mathcal{L}^{r}(E)$ is a Dedekind complete OBA in view of (1.6.7) and ([2], Theorem 3.9).

The following result indicates that there are plenty of examples of Dedekind complete OBAs. The proof of this result is straightforward, and therefore we shall omit it.

Lemma 1.9.5. If $\left(A_{1}, C_{1}\right)$ and $\left(A_{2}, C_{2}\right)$ are Dedekind complete $O B A$ s, then $(A, C)$ is Dedekind complete, where $A:=A_{1} \oplus A_{2}$ and $C:=C_{1} \oplus C_{2}$. 
We remark that, if either $\left(A_{1}, C_{1}\right)$ or $\left(A_{2}, C_{2}\right)$ in Lemma 1.9 .5 is not a Dedekind complete OBA, then $(A, C)$ will not be a Dedekind complete OBA. It is known that $C[0,1]$ (as a Banach lattice, and consequently as an OBA) is not Dedekind complete ([11], Example 2.52). From the implication-scheme on page 15 of this thesis, we deduce now that $C[0,1]$ is not a $K B$-space. This observation will be useful in Example 3.2.8. The space $C(K)$ is Dedekind complete if and only if $K$ is a compact Hausdorff space in which the closure of every open subset of $K$ is open in $K$ ([35], Corollary, p.108). This result provides another reason why $C[0,1]$ is not Dedekind complete.

From this point onward we recall concepts and results from [6] that will be useful in the sequel.

Definition 1.9.6 (Order idempotents). ([6], p.144) Let $(A, C)$ be an OBA. An idempotent $p \in A$ is called an order idempotent if $0 \leq p \leq \mathbf{1}$.

The set of all order idempotents of $A$ will be denoted by $\mathbf{O I}(A)$. Clearly, $p \in \mathbf{O I}(A)$ if and only if $\mathbf{1}-p \in \mathbf{O I}(A)$.

If $V$ denotes a vector lattice and $B$ a projection band in $V$, then $P_{B}: V \rightarrow$ $V$ defined by $P_{B} x=x_{1}$, where $x=x_{1}+x_{2} \in B+B^{d}$, is called an order projection. For a Banach lattice $E$ we have that $T \in \mathbf{O I}(\mathcal{L}(E))$ if and only if $T$ is an order projection on $E$ ([7], Theorem 3.10). It is not difficult to see that $\mathbf{O I}(\mathcal{L}(E))=\mathbf{O I}\left(\mathcal{L}^{r}(E)\right)$

We also have that $\mathbf{O I}(\mathbb{C})=\{0,1\}, \mathbf{O I}\left(\mathbb{C}^{2}\right)=\{(0,0),(1,0),(0,1),(1,1)\}$, $\mathbf{O I}\left(M_{2}(\mathbb{C})\right)=\left\{\left(\begin{array}{ll}0 & 0 \\ 0 & 0\end{array}\right),\left(\begin{array}{ll}1 & 0 \\ 0 & 0\end{array}\right),\left(\begin{array}{ll}0 & 0 \\ 0 & 1\end{array}\right),\left(\begin{array}{ll}1 & 0 \\ 0 & 1\end{array}\right)\right\}=\mathbf{O I}\left(M_{2}^{u}(\mathbb{C})\right), \mathbf{O I}(C[0,1])=\{0, \mathbf{1}\}$ and $\mathbf{O I}\left(l^{\infty}(\mathbb{C})\right)$ consists of $(0,0,0, \ldots),(\mathbf{1}, \mathbf{1}, \ldots)$, together with all sequences consisting of zeros and ones.

The following two results will be needed in proving Theorem 5.5.3.

Lemma 1.9.7. ([6], Lemma 2.1) Let $(A, C)$ be an OBA with proper algebra cone C. If $p_{1}, p_{2} \in \mathbf{O I}(A)$, then $p_{1} p_{2}=p_{2} p_{1}=\inf \left\{p_{1}, p_{2}\right\} \in \mathbf{O I}(A)$.

Corollary 1.9.8. ([6], Corollary 2.2) If $(A, C)$ is a Dedekind complete OBA with proper algebra cone $C$, then $\mathbf{O I}(A)$ is Dedekind complete.

It is familiar that $\mathcal{L}(E)$ is not a Dedekind complete OBA in general (even if $E$ is a Dedekind complete Banach lattice). Nevertheless, since $\mathcal{L}^{r}(E)$ is a Dedekind complete OBA whenever $E$ is a Dedekind complete Banach lattice, we have that $\mathbf{O I}(\mathcal{L}(E))\left(=\mathbf{O I}\left(\mathcal{L}^{r}(E)\right)\right)$ is Dedekind complete in view of Corollary 1.9.8. 
Definition 1.9.9 (a-invariant). ([6], p.144) Let $(A, C)$ be an $O B A$ and $a \in A$. An element $p \in \mathbf{O I}(A)$ is said to be a-invariant if $(\mathbf{1}-p)$ ap $=0$.

Definition 1.9.10 (a-invariant chain). ([6], p.147) Let $(A, C)$ be an OBA and $a \in A$. A simply ordered set of order idempotents $\left\{p_{n}, \ldots, p_{1}\right\}$ is called an ainvariant chain if $p_{n} \geq \cdots \geq p_{1}$ and $p_{i}$ is a-invariant for each $i \in\{1, \ldots, n\}$.

Analogously, if $T$ is a bounded linear operator on a Banach lattice, then a simply ordered set of $T$-invariant projection bands $\left\{B_{n}, \ldots, B_{1}\right\}$ is called a $T$-invariant chain if $B_{n} \supseteq \cdots \supseteq B_{1}$.

Definition 1.9.11 (Irreducible elements). ([6], p.144) Let $(A, C)$ be an OBA. An element $a \in C$ is said to be irreducible if $p=0$ and $p=\mathbf{1}$ are the only a-invariant order idempotents.

If $E$ is a Dedekind complete Banach lattice, then $0 \leq T \in \mathcal{L}(E)$ is a band irreducible operator on $E$ if and only if $T$ is an irreducible element in $\mathcal{L}(E)$; that is, $E$ contains no non-trivial $T$-invariant (projection) bands if and only if $T$ has no non-trivial $T$-invariant order projections ([6], p.144).

We mention that every non-zero positive element in an OBA $(A, C)$ is irreducible if and only if $\mathbf{O I}(A)=\{0, \mathbf{1}\}$ ([6], p.158).

Definition 1.9.12 (Irreducible w.r.t. an order idempotent). ([6], p.144) Let $(A, C)$ be an $\mathrm{OBA}$ and $0<p \in \mathbf{O I}(A)$. An element $a \in C$ is said to be irreducible w.r.t. $p$ if there exists no $p^{*} \in \mathbf{O I}(A)$ such that $0<p^{*}<p$ and $\left(p-p^{*}\right) a p^{*}=0$.

It is easy to check that a positive OBA element $a$ is irreducible if and only if $a$ is irreducible w.r.t. the unit in the OBA.

Definition 1.9.13 (Order continuous element). ([6], p.150) Let $(A, C)$ be an $O B A$. An element $a \in C$ is said to be order continuous if $p_{\alpha} a \downarrow 0$ and $a p_{\alpha} \downarrow 0$ whenever $p_{\alpha} \downarrow 0$, where $\left(p_{\alpha}\right)$ is a net in $\mathbf{O I}(A)$.

Let $A_{n}$ denote the set of all order continuous elements of an $\operatorname{OBA}(A, C)$. It then follows that, if $0 \leq a \leq b$ relative to (a proper algebra cone) $C$, then $b \in A_{n}$ implies that $a \in A_{n}$.

It is easy to verify that $\mathbb{C}_{n}=\mathbb{R}^{+},\left(\mathbb{C}^{2}\right)_{n}=\mathbb{R}^{2+},\left(l^{\infty}(\mathbb{C})\right)_{n}=l^{\infty}\left(\mathbb{R}^{+}\right)$and $\left(M_{2}^{u}(\mathbb{C})\right)_{n}=M_{2}^{u}\left(\mathbb{R}^{+}\right)$. In general, if $\mathbf{O I}(A)=\{0, \mathbf{1}\}$ for some OBA $(A, C)$, then $A_{n}=C$. Note that $\mathbf{O I}(A)=\{0, \mathbf{1}\}$ is not a necessary condition for $A_{n}=C$ as can be seen in the cases of $\mathbb{C}^{2}, l^{\infty}(\mathbb{C})$ and $M_{2}^{u}(\mathbb{C})$ (recalling the examples preceding Lemma 1.9.7). 
An immediate consequence of the fact that $\left.\mathbf{O I}(\mathcal{L}(E))=\mathbf{O I}\left(\mathcal{L}^{r}(E)\right)\right)$ is the identity $(\mathcal{L}(E))_{n}=\left(\mathcal{L}^{r}(E)\right)_{n}$. We remark that an order continuous operator on a Banach lattice $E$ is not necessarily an order continuous element in $\mathcal{L}(E)$ ([6], p.150). However, if $E$ is a Dedekind complete Banach lattice, then the set of all order continuous elements of $\mathcal{L}(E)$ coincides with the set of all positive order continuous operators on $E$ ([6], Example 2.9(a)). Moreover, if $E$ is a Banach lattice with order continuous norm, then by ([6], p.150) we have that $(\mathcal{L}(E))_{n}=\left\{T \in \mathcal{L}(E): T E_{+} \subseteq E_{+}\right\}$.

From this point on we use the notation $a_{p}$ to mean pap and $p^{d}$ to mean $\mathbf{1}-p$, where $p$ is an order idempotent and $a$ an element in an ordered Banach algebra.

Definition 1.9.14 (Block). ([6], p.150) Let $(A, C)$ be an $O B A$ and $a \in C$. An element $b \in A$ is called a block of $a$ if there exists an a-invariant chain $\left\{p_{2}, p_{1}\right\}$, with $p_{2}>p_{1}$, such that $b=a_{p_{2} p_{1}^{d}}$.

Clearly, if $b$ is a block of $a$, then $0 \leq b \leq a$. From ([6], Lemma 2.6) it then follows, under the additional assumption that the algebra cone $C$ is proper, that $r(b) \leq r(a)$.

Definition 1.9.15 (Spectral block). ([6], p.150) Let $(A, C)$ be an OBA with proper algebra cone $C$ and $a \in C$. An element $b \in A$ is called a spectral block of $a$ whenever $b$ is a block of $a$ and $r(a)=r(b)$.

Obviously, $a \in C$ is a spectral block of itself.

Definition 1.9.16 (Spectrally order continuous element). ([6], p.150) Let $(A, C)$ be an $O B A$ with proper algebra cone $C$. An order continuous element $a \in C$ is said to be spectrally order continuous if, for every spectral block $b$ of $a$, the condition that $r(b)$ is a pole of order $m$ of $(\lambda \mathbf{1}-b)^{-1}$ implies that $b_{-m} \in A_{n}$.

A positive operator $T$ on a Banach lattice $E$ is called a spectrally order continuous operator if $T$ is an order continuous operator on $E$ and if, for every spectral block $S$ of $T$, the condition that $r(S)$ is a pole of order $m$ of $(\lambda \mathbf{I}-S)^{-1}$ implies that $S_{-m}$ is an order continuous operator on $E([6]$, p.150). Again, the notion of a spectrally order continuous operator on $E$ differs from the notion of a spectrally order continuous element in $\mathcal{L}(E)$. However, if $E$ is Dedekind complete and $0 \leq T \in \mathcal{L}(E)$, then $T$ is a spectrally order continuous operator on $E$ if and only if $T$ is a spectrally order continuous element in $\mathcal{L}(E)([6]$, p.151). 
We state the following remark for use in Corollary 5.5.8.

Remark 1.9.17. Let $E$ be a Banach lattice and $0 \leq T \in \mathcal{L}(E)$. If $T$ is spectrally order continuous in $\mathcal{L}(E)$, then $T$ is spectrally order continuous in $\mathcal{L}^{r}(E)$.

To establish the above implication, we observe that the equality $\mathbf{O I}(\mathcal{L}(E))$ $\left.=\mathbf{O I}\left(\mathcal{L}^{r}(E)\right)\right)$ implies that the set of blocks of $T$ in $\mathcal{L}(E)$ and the set of blocks of $T$ in $\mathcal{L}^{r}(E)$ coincide. By also using ([36], p.79), which asserts that $r\left(T, \mathcal{L}^{r}(E)\right)=r(T, \mathcal{L}(E))$ for all $0 \leq T \in \mathcal{L}(E)$, it follows that the set of spectral blocks of $T$ in $\mathcal{L}(E)$ and the set of spectral blocks of $T$ in $\mathcal{L}^{r}(E)$ coincide. By the uniqueness of the Laurent series the condition " $r(T)$ is a pole of order, say, $m$ of $(\lambda I-T)^{-1}$ in $\mathcal{L}^{r}(E)$ " implies that " $r(T)$ is a pole of order $m$ of $(\lambda I-T)^{-1}$ in $\mathcal{L}(E)^{\prime}$. It remains to recall the identity $(\mathcal{L}(E))_{n}=\left(\mathcal{L}^{r}(E)\right)_{n}$ which was discussed before.

The following result will be required in Corollary 5.5.9.

Proposition 1.9.18. Let $(A, C)$ be an $O B A$ with closed and proper algebra cone $C$ such that $A_{n}=C$. Then $a \in A_{n}$ if and only if $a$ is spectrally order continuous.

Hence, every positive element is spectrally order continuous.

Proof. For the non-trivial implication suppose that $a \in A_{n}$. Let $b$ be a spectral block of $a$ such that $r(b)$ is a pole of order $m$ of $(\lambda \mathbf{1}-b)^{-1}$. From the definition of a spectral block it follows that $b \in C$, and hence $b_{-m} \in C=A_{n}$ in view of Theorem 1.8.1 and assumption.

We note that, for the examples given below Definition 1.9.13, every positive element is spectrally order continuous.

The following fact will be useful in the sequel. It was mentioned in ([5], Lemma 6.1 and the remark thereafter) - see also ([6], p.152).

Lemma 1.9.19. Let $E$ be an AM-space with unit. If $T$ is a positive order continuous operator on $E$, then $T$ is a spectrally order continuous operator on $E$.

Definition 1.9.20 (Disjunctive product). ([6], p.158) An OBA $(A, C)$ is said to have a disjunctive product if for any $a, b \in A_{n}$ with $a b=0$ there exists $p \in \mathbf{O I}(A)$ satisfying ap $=0=(\mathbf{1}-p) b$.

Since $\mathbf{O I}(\mathcal{L}(E))=\mathbf{O I}\left(\mathcal{L}^{r}(E)\right)$ (and therefore $\left.(\mathcal{L}(E))_{n}=\left(\mathcal{L}^{r}(E)\right)_{n}\right)$, it follows that $\mathcal{L}(E)$ has a disjunctive product if and only if $\mathcal{L}^{r}(E)$ has a disjunctive product. If $E$ is a Dedekind complete Banach lattice, then from ([6], Example $3.3(\mathrm{a})$ ) we have that $\mathcal{L}(E)$ has a disjunctive product. 
More examples of OBAs having disjunctive products include $\left(\mathbb{C}, \mathbb{R}^{+}\right)$(or more generally $\left.\left(\mathbb{C}^{2}, \mathbb{R}^{2+}\right)\right),\left(l^{\infty}(\mathbb{C}), l^{\infty}\left(\mathbb{R}^{+}\right)\right)$and $\left(M_{n}^{u}(\mathbb{C}), M_{n}^{u}\left(\mathbb{R}^{+}\right)\right)$.

Definition 1.9.21 ( $f$-pole). ([6], p.152) Let $(A, C)$ be an $O B A$ and $b \in C$. Then $r(b)$ is called an $f$-pole of $(\lambda \mathbf{1}-b)^{-1}$ if $0 \leq a \leq b$ implies that $r(a) \leq r(b)$, and if $r(a)=r(b)$ implies that $r(a)$ is a pole of $(\lambda \mathbf{1}-a)^{-1}$.

Our next result states conditions under which, given that the spectral radius $r(a)$ of a positive OBA element $a$ is a Riesz point of the spectrum of $a$, then $r(a)$ is an $f$-pole of the resolvent of $a$.

Proposition 1.9.22. Let $(A, C)$ be a semisimple OBA with closed algebra cone $C$ such that the spectral radius function in $(A, C)$ is monotone and let $I$ be a closed inessential ideal such that the spectral radius function in $(A / I, \pi C)$ is weakly monotone. If $b \in C$ is such that $r(b)$ is a Riesz point of $\sigma(b)$ relative to $I$, then $r(b)$ is an $f$-pole of $(\lambda \mathbf{1}-b)^{-1}$.

Proof. The statement $0 \leq a \leq b$ implies $r(a) \leq r(b)$ follows from the fact that the spectral radius function in $(A, C)$ is monotone. Consider the case where $r(a)=r(b)$. By Theorem 1.8.5 we have that $r(a)$ is a Riesz point of $\sigma(a)$ relative to $I$, and hence $r(a)$ is a pole of $(\lambda \mathbf{1}-a)^{-1}$ by Lemma 1.4.5. Thus $r(b)$ is an $f$-pole of $(\lambda \mathbf{1}-b)^{-1}$ by definition.

For an OBA $(A, C)$ the inequalites $0 \leq a_{p} \leq a$ clearly hold for all $p \in$ $\mathbf{O I}(A)$ and $a \in C$. By ([6], Lemma 2.4), if $\left\{\mathbf{1}=p_{n}, p_{n-1}, \ldots, p_{1}, p_{0}=0\right\}$ is an $a$-invariant chain, then $\sigma(a) \subseteq \cup_{i=1}^{n} \sigma\left(a_{p_{i} p_{i-1}^{d}}\right)$. Therefore, there exists $i \in\{1, \ldots, n\}$ such that $r(a) \leq r\left(a_{p_{i} p_{i-1}^{d}}\right)$. Hence we note that, under the additional assumption that the algebra cone $C$ is proper, the identity $r(a)=$ $r\left(a_{p_{i_{0}} p_{i_{0}-1}^{d}}\right)$ always holds for some $i_{0} \in\{1, \ldots, n\}$ in view of ([6], Lemma 2.6).

Definition 1.9.23 (Frobenius normal form). ([6], p.154) Let $(A, C)$ be an OBA and $a \in C$. Then $a$ is said to have the Frobenius normal form if there exists an a-invariant chain $\mathscr{C}=\left\{\mathbf{1}=p_{n}, p_{n-1}, \ldots, p_{1}, p_{0}=0\right\}$ such that, if $r\left(a_{p_{i} p_{i-1}^{d}}\right)=$ $r(a)$ for some $i \in\{1, \ldots, n\}$, then $a_{p_{i} p_{i-1}^{d}}$ is irreducible w.r.t. $p_{i} p_{i-1}^{d}$.

We say that $\mathscr{C}$ determines the Frobenius normal form of $a$.

Let $\left\{E=B_{n}, B_{n-1}, \ldots, B_{1}, B_{0}=\{0\}\right\}$ be a $T$-invariant chain of projection bands, where $T$ is a positive bounded linear operator on a Dedekind complete Banach lattice $E$, and $Q_{i}:=B_{i} \cap B_{i-1}^{d}$. From ([7], Theorem 3.11[(1) and 
(2)]) the identities

$$
P_{Q_{i}} T P_{Q_{i}}=P_{B_{i} \cap B_{i-1}^{d}} T P_{B_{i} \cap B_{i-1}^{d}}=P_{B_{i}}\left(I-P_{B_{i-1}}\right) T P_{B_{i}}\left(I-P_{B_{i-1}}\right)
$$

hold. Moreover, in view of the remark following Definition 1.9.11 and ([7], Theorem 3.12), Definition 1.9.23 can be made more precise in the case where $A=\mathcal{L}(E)$. A straightforward argument shows that $P_{Q_{i}} T P_{Q_{i}}$ is irreducible w.r.t. $P_{Q_{i}}$ if and only if the restriction of $P_{Q_{i}} T P_{Q_{i}}$ to $Q_{i}$ is band irreducible. These observations are summarized in the following remark.

Remark 1.9.25. Let $E$ be a Dedekind complete Banach lattice. An operator $0 \leq$ $T \in \mathcal{L}(E)$ has the Frobenius normal form if there exists a T-invariant chain $\{E=$ $\left.B_{n}, B_{n-1}, \ldots, B_{1}, B_{0}=\{0\}\right\}$ of projection bands such that, if $r\left(P_{Q_{i}} T P_{Q_{i}}\right)=r(T)$ for some $i \in\{1, \ldots, n\}$, then the restriction of $P_{Q_{i}} T P_{Q_{i}}$ to $Q_{i}$ is band irreducible, where $Q_{i}:=B_{i} \cap B_{i-1}^{d}$.

Results such as ([4], Theorem 13) and ([6], Corollaries 2.12 and 2.13 and Theorem 2.14) give sufficient conditions for the existence of the Frobenius normal form of a bounded linear operator on a Banach lattice.

For a Banach lattice $E$ it is easy to verify that, for a positive operator $T$ on $E$, we have that $T$ has the Frobenius normal form in $\mathcal{L}^{r}(E)$ if and only if $T$ has the Frobenius normal form in $\mathcal{L}(E)$.

Theorem 1.9.26. ([6], Theorem 2.11) Let $(A, C)$ be a Dedekind complete OBA with closed and proper algebra cone $C$. If $a \in C$ is a spectrally order continuous element such that $r(a)>0$ and $r(a)$ is an f-pole of $(\lambda \mathbf{1}-a)^{-1}$, then a has the Frobenius normal form.

Theorem 1.9.26 illustrates that, under certain natural conditions, the spectrum of a positive (spectrally order continuous) element in an OBA is determined by the spectra of certain associated irreducible elements.

Finally, the following result will be needed in Section 5.5.

Lemma 1.9.27. ([6], Lemma 5.2) Let $(A, C)$ be an $O B A$ which has a disjunctive product with closed and proper algebra cone $C$ such that $\mathbf{O I}(A)$ is Dedekind complete, $0<p \in \mathbf{O I}(A)$ and $a \in A$. Suppose that the following conditions hold:

(a) $0<a_{p} \in A_{n}$ is irreducible w.r.t. $p$,

(b) $r\left(a_{p}\right)$ is a pole of order $m$ of $\left(\lambda \mathbf{1}-a_{p}\right)^{-1}$ and

(c) $\left(a_{p}\right)_{-m} \in A_{n}$.

Then $r\left(a_{p}\right)>0$ and $r\left(a_{p}\right)$ is a simple pole of $\left(\lambda \mathbf{1}-a_{p}\right)^{-1}$. 


\section{Chapter 2}

\section{Poles and the Laurent expansion of the resolvent}

In this chapter, suppose that $R(\lambda, a)=\sum_{n=-\infty}^{\infty}\left(\lambda-\lambda_{0}\right)^{n} a_{n}$ is the Laurent expansion of $(\lambda \mathbf{1}-a)^{-1}$ in a deleted neighbourhood of $\lambda_{0} \in$ iso $\sigma(a)$, where $a$ is an element of a Banach algebra $A$. Throughout this work we will frequently refer to the coefficients $a_{k}(k \in \mathbb{Z})$ of $\left(\lambda-\lambda_{0}\right)^{k}$ in the above Laurent series expansion. It is known that the spectral idempotent of $a$ corresponding to $\lambda_{0}$ is the coefficient $a_{-1}$.

The aim of Section 2.1 is to summarize several results (stemming from previous facts) that deal with ideals and poles that will be useful in the sequel. Subsequently, we present, in Section 2.2, some properties of $a_{k}$ that are known to hold in the case $A=\mathcal{L}(X)$, with $X$ a Banach space. We point out here that the proofs given in this section depend mainly on the holomorphic functional calculus and we include them for the sake of completeness.

It is worth mentioning that the results included in this chapter will be employed in Chapter 5.

\subsection{Ideals, homomorphisms and poles}

We begin this section with the following theorem which describes the relationship between the Fredholm spectrum of an element $a \in A$ relative to a Banach algebra homomorphism $T: A \rightarrow B$ with closed range and the Fredholm spectrum of $a$ relative to the canonical homomorphism $\pi: A \rightarrow A / \bar{I}$, where $I$ is an arbitrary ideal of $A$. In order to prove this result, we use an approach similar to that of H. Mouton, S. Mouton and H. Raubenheimer in 
([28], Theorem 6.4(1)). This result is of independent interest.

Theorem 2.1.1. Let $T: A \rightarrow B$ be a Banach algebra homomorphism with closed range and let $I$ be an ideal of $A$. Then $\sigma(a+\bar{I}) \subseteq \eta \sigma(T a)$ for all $a \in A$.

In particular, if $T$ is onto, then $\sigma(a+\bar{I}) \subseteq \sigma(T a)$.

Proof. Since $T$ has closed range, $T(A) \subseteq B$ is a Banach algebra which contains the unit of $B$. Consider the homomorphism $\phi: T(A) \rightarrow A / \bar{I}$ defined by $\phi(T a)=a+\bar{I}$ for all $a \in A$. Then, by using Theorem 1.2.2(ii), we have that

$$
\sigma(a+\bar{I})=\sigma(\phi(T a)) \subseteq \sigma(T a, T(A)) \subseteq \eta \sigma(T a, T(A))=\eta \sigma(T a, B)
$$

for all $a \in A$.

Now suppose that $T$ is onto. Then $T(A)=B$, and the result is clear from the first inclusion in (2.1.2).

From this point on we present useful consequences of preceding results. Using Theorem 2.1.1 we can state the following result for bounded Banach algebra homomorphisms with closed range. We remind the reader of Remark 1.1.4.

Corollary 2.1.3. Let $T: A \rightarrow B$ be a bounded Banach algebra homomorphism with closed range. If $a \in A$, then $\eta \sigma(T a)=\eta \sigma(\pi a)$.

Proof. Since $T=T^{*} \circ \pi$, where the homomorphism $T^{*}: A / \mathrm{N}(T) \rightarrow B$ is defined by $T^{*}(a+\mathrm{N}(T))=T a$ and $\pi: A \rightarrow A / \overline{\mathrm{N}(T)}$ is the canonical homomorphism, the inclusion $\sigma(T a) \subseteq \sigma(a+\mathrm{N}(T))=\sigma(\pi a)$ is clear. From Theorem 2.1.1 we have that $\sigma(\pi a) \subseteq \eta \sigma(T a)$ for all $a \in A$, and hence the result follows.

If we replace the statement " $T$ is bounded" in Corollary 2.1.3 by the statement " $T$ has the Riesz property", then we obtain the following similar result. We mention here that, without the assumption that $\mathrm{N}(T)$ is an inessential ideal, the set $\eta D(a, \mathrm{~N}(T)$ ) (in view of Theorem 1.4.6) cannot in general be added to the list in Corollary 2.1.3.

Corollary 2.1.4. Let $T: A \rightarrow B$ be a Banach algebra homomorphism with closed range satisfying the Riesz property. If $a \in A$, then $\eta \sigma(T a)=\eta D(a, \mathrm{~N}(T))=$ $\eta \sigma(\pi a)$. 
Proof. Using Theorem 1.4.6, Theorems 1.5.7 and 1.5.8 and ([29], Corollary 5.4) we have that $\eta \sigma(T a)=\eta \beta_{T}(a)=\eta \beta_{\pi}(a)=\eta D(a, \mathrm{~N}(T))=\eta \sigma(\pi a)$ for all $a \in A$.

The following result will frequently be referred to. This result summarizes equivalent conditions for $r(a)$ to be a Riesz point of $\sigma(a)$ relative to $\mathrm{N}(T)$.

Corollary 2.1.5. Let $T: A \rightarrow B$ be a Banach algebra homomorphism with closed range satisfying the Riesz property and $a \in A$. Then the following statements are equivalent:

(i) $r(a) \in \sigma(a) \backslash \sigma(T a)$.

(ii) $r(a) \in \sigma(a) \backslash \sigma(a+\overline{\mathrm{N}(T)})$.

(iii) $r(a)$ is a Riesz point of $\sigma(a)$ relative to $\mathrm{N}(T)$.

Moreover, if $A$ is a semisimple Banach algebra, then $r(a) \in \sigma(a) \backslash \sigma(T a)$ if and only if $r(a)>0$ is a pole of $(\lambda \mathbf{1}-a)^{-1}$ and $p(a, r(a)) \in \mathrm{N}(T)$.

Proof. The equivalences (i) through (iii) follow from Corollary 2.1.4, while the last part, under the additional assumption that $A$ is semisimple, is clear from Lemma 1.4.5.

In view of Corollary 2.1.3 the equivalence of (i) and (ii) in Corollary 2.1.5 still holds whenever the condition " $T$ has the Riesz property" is replaced by the statement " $T$ is bounded".

Finally, we state and prove the following observation regarding the Frobenius normal form that will be useful in Section 5.5.

Corollary 2.1.6. Let $(A, C)$ be a Dedekind complete semisimple OBA with closed and proper algebra cone $C$ such that the spectral radius function in $(A, C)$ is monotone. Also, suppose that $T: A \rightarrow B$ is a Banach algebra homomorphism with closed range satisfying the Riesz property such that the spectral radius function in $(A / \overline{\mathrm{N}}(T), \pi C)$ is weakly monotone. If $a \in C$ is a spectrally order continuous element such that $r(a) \notin \sigma(\mathrm{Ta})$, then a has the Frobenius normal form.

Proof. In order to apply Theorem 1.9.26, we show that $r(a)$ is an $f$-pole of $(\lambda \mathbf{1}-a)^{-1}$. We do so by verifying that all conditions in Proposition 1.9.22 are satisfied.

First we point out that $\overline{\mathrm{N}(T)}$ is a closed inessential ideal of $A$. This statement follows from the fact that $T$ satisfies the Riesz property. 
Using Theorem 1.8.2 we have that $r(a) \in \sigma(a)$, and hence $r(a)$ is a Riesz point of $\sigma(a)$ relative to $\overline{\mathrm{N}(T)}$ in view of Corollary 2.1.5. By Proposition 1.9.22 we have that $r(a)>0$ is an $f$-pole of $(\lambda \mathbf{1}-a)^{-1}$, and hence the Frobenius normal form of $a$ exists by Theorem 1.9.26.

\subsection{Coefficients of Laurent series}

In this section we present some properties of the coefficients of the main part of the Laurent series of the resolvent that will be useful in the sequel.

The following result which we establish here in the setting of Banach algebras were stated in [3] for bounded linear operators on Banach lattices.

Lemma 2.2.1. Let $A$ be a Banach algebra, $a \in A$ and $\lambda_{0} \in$ iso $\sigma(a)$. Then, if the coefficients in the Laurent expansion of $R(\lambda, a)$ in a neighbourhood of $\lambda_{0}$ are indicated by $a_{n}$, we have the following identities:

(a) $a_{-j} a_{-i}=a_{-i} a_{-j}=a_{-i-j+1}$ for all $i, j \geq 1$.

(b) $a_{-j} a_{i}=a_{i} a_{-j}=0$ for all $i \geq 0$ and $j \geq 1$.

Proof. (a) Let $U_{1}$ and $U_{0}$ be disjoint open sets such that $U_{1}$ contains $\left\{\lambda_{0}\right\}$ and $U_{0}$ contains $\sigma(a) \backslash\left\{\lambda_{0}\right\}$. Choose $\Gamma_{1}$ to be a small circle in $U_{1}$ surrounding $\left\{\lambda_{0}\right\}$ and let $\Gamma_{2}$ be a smooth contour in $U_{0}$ surrounding $\sigma(a) \backslash\left\{\lambda_{0}\right\}$. Define, for $j \geq 1$, the function $f_{j}: U_{0} \cup U_{1} \rightarrow \mathbb{C}$ by

$$
f_{j}(\lambda)=\left\{\begin{array}{cl}
0 & \text { if } \lambda \in U_{0} \\
\left(\lambda-\lambda_{0}\right)^{j-1} & \text { if } \lambda \in U_{1}
\end{array}\right.
$$

Then $f_{j} \in H\left(U_{0} \cup U_{1}\right)$ and by Proposition 1.3.1

$$
\begin{aligned}
f_{j}(a) & =\frac{1}{2 \pi i} \int_{\Gamma_{1} \cup \Gamma_{2}} f_{j}(\lambda)(\lambda \mathbf{1}-a)^{-1} d \lambda \\
& =\frac{1}{2 \pi i} \int_{\Gamma_{1}}\left(\lambda-\lambda_{0}\right)^{j-1}(\lambda \mathbf{1}-a)^{-1} d \lambda .
\end{aligned}
$$

The coefficients $a_{n}$ are given by

$$
a_{n}=\frac{1}{2 \pi i} \int_{\Gamma_{1}} \frac{(\lambda \mathbf{1}-a)^{-1}}{\left(\lambda-\lambda_{0}\right)^{n+1}} d \lambda
$$

Therefore

$$
a_{-j}=\frac{1}{2 \pi i} \int_{\Gamma_{1}}\left(\lambda-\lambda_{0}\right)^{j-1}(\lambda \mathbf{1}-a)^{-1} d \lambda=f_{j}(a)
$$


for all $j \geq 1$ and, by also using Proposition 1.3.1 and Theorem 1.3.2(2),

$$
\begin{aligned}
a_{-i-j+1} & =\frac{1}{2 \pi i} \int_{\Gamma_{1}}\left(\lambda-\lambda_{0}\right)^{i+j-1-1}(\lambda \mathbf{1}-a)^{-1} d \lambda \\
& =\frac{1}{2 \pi i} \int_{\Gamma_{1} \cup \Gamma_{2}}\left(f_{i} f_{j}\right)(\lambda)(\lambda \mathbf{1}-a)^{-1} d \lambda \\
& =\left(f_{i} f_{j}\right)(a) \\
& =f_{i}(a) f_{j}(a)\left(=f_{j}(a) f_{i}(a)\right) \\
& =a_{-i} a_{-j}\left(=a_{-j} a_{-i}\right)
\end{aligned}
$$

for all $i \geq 1$.

(b) Following (a), let $\Lambda_{1}$ be another circle with centre $\lambda_{0}$ such that $\Lambda_{1}$ lies inside the interior of $\Gamma_{1}$. Also, let $i \geq 0$ and $j \geq 1$. Then, using the resolvent equation and Cauchy's integral and generalized integral formulas, we have that

$$
\begin{aligned}
a_{i} a_{-j}= & \left(\frac{1}{2 \pi i} \int_{\Lambda_{1}} \frac{(\mu \mathbf{1}-a)^{-1}}{\left(\mu-\lambda_{0}\right)^{i+1}} d \mu\right)\left(\frac{1}{2 \pi i} \int_{\Gamma_{1}} \frac{(\lambda \mathbf{1}-a)^{-1}}{\left(\lambda-\lambda_{0}\right)^{-j+1}} d \lambda\right) \\
= & \left(\frac{1}{2 \pi i}\right)^{2} \int_{\Lambda_{1}}\left[\int_{\Gamma_{1}}\left(\lambda-\lambda_{0}\right)^{j-1}\left(\mu-\lambda_{0}\right)^{-i-1}(\lambda \mathbf{1}-a)^{-1}(\mu \mathbf{1}-a)^{-1} d \lambda\right] d \mu \\
= & \left(\frac{1}{2 \pi i}\right) \int_{\Lambda_{1}}^{2}\left[\int_{\Gamma_{1}}\left(\lambda-\lambda_{0}\right)^{j-1}\left(\mu-\lambda_{0}\right)^{-i-1}\left(\frac{(\lambda \mathbf{1}-a)^{-1}-(\mu \mathbf{1}-a)^{-1}}{\mu-\lambda}\right) d \lambda\right] d \mu \\
= & \frac{1}{2 \pi i} \int_{\Gamma_{1}}\left(\lambda-\lambda_{0}\right)^{j-1}\left[\frac{1}{2 \pi i} \int_{\Lambda_{1}} \frac{\left(\mu-\lambda_{0}\right)^{-i-1}}{\mu-\lambda} d \mu\right](\lambda \mathbf{1}-a)^{-1} d \lambda \\
& \quad-\frac{1}{2 \pi i} \int_{\Lambda_{1}}\left(\mu-\lambda_{0}\right)^{-i-1}\left[\frac{1}{2 \pi i} \int_{\Gamma_{1}} \frac{\left(\lambda-\lambda_{0}\right)^{j-1}}{\mu-\lambda} d \lambda\right](\mu \mathbf{1}-a)^{-1} d \mu \\
= & \frac{1}{2 \pi i} \int_{\Gamma_{1}}\left(\lambda-\lambda_{0}\right)^{j-1} \frac{f^{(i)}\left(\lambda_{0}\right)}{i !}(\lambda \mathbf{1}-a)^{-1} d \lambda \\
& \quad+\frac{1}{2 \pi i} \int_{\Lambda_{1}}\left(\mu-\lambda_{0}\right)^{-i-1} g(\mu)(\mu \mathbf{1}-a)^{-1} d \mu,
\end{aligned}
$$

where the functions $f$ and $g$ are chosen to be $f(\mu)=\frac{1}{\mu-\lambda}$ and $g(\lambda)=(\lambda-$ $\left.\lambda_{0}\right)^{j-1}$. Using (2.2.3) and the fact that $f^{(i)}\left(\lambda_{0}\right)=(-1)^{i} i !\left(\lambda_{0}-\lambda\right)^{-(i+1)}$, we get that

$$
\begin{aligned}
a_{i} a_{-j}= & -\frac{1}{2 \pi i} \int_{\Gamma_{1}}\left(\lambda-\lambda_{0}\right)^{j-i-1-1}(\lambda \mathbf{1}-a)^{-1} d \lambda \\
& \quad+\frac{1}{2 \pi i} \int_{\Lambda_{1}}\left(\mu-\lambda_{0}\right)^{j-i-1-1}(\mu \mathbf{1}-a)^{-1} d \mu \\
= & -a_{i-j+1}+a_{i-j+1}=0 .
\end{aligned}
$$


The following result will be useful in our work.

Corollary 2.2.5. Let $A$ be a Banach algebra, $a \in A$ and $\lambda_{0} \in$ iso $\sigma(a)$. If $k>1$, then $a_{-k} \in \mathrm{QN}(A) \cap \operatorname{Comm}^{2}(a)$.

Proof. Let $k>1$. By (2.2.4) we have that $a_{-k}=f_{k}(a)$, where $f_{k}$ is defined as in (2.2.2). Hence $a_{-k} \in \operatorname{Comm}^{2}(a)$. Using the spectral mapping theorem we have that

$$
\sigma\left(a_{-k}\right)=\sigma\left(f_{k}(a)\right)=f_{k}(\sigma(a))=\left\{f_{k}(\lambda): \lambda \in \sigma(a)\right\}=\{0\}
$$

that is, $a_{-k} \in \mathrm{QN}(A)$.

We point out the following well-known fact for ease of reference.

Corollary 2.2.6. Let $A$ be a Banach algebra, $a \in A$ and $\lambda_{0} \in$ iso $\sigma(a)$. Then $\sigma\left(a \pm a_{-1}\right)=\left(\sigma(a) \backslash\left\{\lambda_{0}\right\}\right) \cup\left\{\lambda_{0} \pm 1\right\}$.

Proof. Choosing $U_{0}$ and $U_{1}$ as in the proof of Lemma 2.2.1 and $f_{1}$ as described in (2.2.2), we define $g: U_{0} \cup U_{1} \rightarrow \mathbb{C}$ by $g(\lambda)=\lambda+f_{1}(\lambda)$ for all $\lambda \in U_{0} \cup U_{1}$.

Then $g \in H\left(U_{0} \cup U_{1}\right)$ and by using Proposition 1.3.1, the spectral mapping theorem and equation (2.2.4) we have that

$$
\sigma\left(a+a_{-1}\right)=\sigma(g(a))=g(\sigma(a))=\left(\sigma(a) \backslash\left\{\lambda_{0}\right\}\right) \cup\left\{\lambda_{0}+1\right\} .
$$

The proof of the identity $\sigma\left(a-a_{-1}\right)=\left(\sigma(a) \backslash\left\{\lambda_{0}\right\}\right) \cup\left\{\lambda_{0}-1\right\}$ is done in a similar manner.

We note the following result which is frequently used throughout this thesis.

Lemma 2.2.7. Let $T: A \rightarrow B$ be a Banach algebra homomorphism, $a \in A$ and $\lambda_{0} \in($ iso $\sigma(a)) \backslash \sigma(T a)$. Then $a_{-i} \in \mathrm{N}(T)$ for all $i \geq 1$.

Proof. It can be easily verified that the condition $\lambda_{0} \in($ iso $\sigma(a)) \backslash \sigma(T a)$ implies that $\lambda_{0} \mathbf{1}-a \in A^{D} \cap \mathcal{F}_{T}$. By Proposition 1.5.5 and Lemma 1.3.4 we have that $a_{-1}=p\left(a, \lambda_{0}\right)=p\left(\lambda_{0} \mathbf{1}-a, 0\right) \in \mathrm{N}(T)$.

If $i>1$, then by taking Lemma 2.2.1(a) into account it follows that $a_{-i}=$ $a_{-i-1+1}=a_{-i} a_{-1} \in \mathrm{N}(T)$. The proof is done. 
We conclude this section with two useful results wherein we identify certain types of OBAs in which (if the spectral radius of a (positive) element $a$ is a pole of the resolvent of $a$ ), the spectral radius of $a$ will always be a simple pole of the resolvent of $a$.

Lemma 2.2.8. Let $A$ be a commutative semisimple Banach algebra and $a \in A$. If $r(a)$ is a pole of $(\lambda \mathbf{1}-a)^{-1}$, then it is a simple pole.

Proof. Assume by way of contradiction that $r(a)$ is a pole of order $k>1$ of $(\lambda \mathbf{1}-a)^{-1}$. Then $a_{-k} \neq 0$, and $a_{-k} \in \mathrm{QN}(A)$ follows from Corollary 2.2.5. Since $A$ is a commutative and semisimple Banach algebra, we have that $\{0\}=\operatorname{Rad}(A)=\mathrm{QN}(A)$, and hence $a_{-k}=0$. Consequently $r(a)$ is a simple pole of $(\lambda \mathbf{1}-a)^{-1}$.

Lemma 2.2.9. Let $(A, C)$ be an $O B A$ with proper, closed and inverse-closed algebra cone $C$ and $a \in C$. If $r(a)$ is a pole of $(\lambda \mathbf{1}-a)^{-1}$, then it is a simple pole.

Proof. Suppose this is not true and let $r(a)$ be a pole of order $k>1$ of $(\lambda \mathbf{1}-a)^{-1}$. From Theorem 1.8.1 and Corollary 2.2.5 we have that $a_{-k} \in$ $C \cap \mathrm{QN}(A)$. Using Corollary 1.8.8 it follows that $a_{-k}=0$. But this is a contradiction to the definition of a pole of order $k$. Hence $r(a)$ is a simple pole of $(\lambda \mathbf{1}-a)^{-1}$. 


\section{Chapter 3}

\section{Upper Browder and upper Weyl elements}

Although the general Fredholm theory in Banach algebras has been widely studied by several authors, its interplay with the theory of positivity in OBAs has not been given much attention yet.

For a Banach lattice E, Alekhno ([4], Theorem 3) discovered that

$$
\mathcal{L}(E)^{-1}+\mathcal{K}(E)=\mathcal{W}_{\pi}=\mathcal{L}(E)^{-1}+(K \cap \mathcal{K}(E)),
$$

where $K$ denotes the cone of positive operators on $E$. Motivated by this discovery, we introduce and investigate the set $A^{-1}+(C \cap \mathrm{N}(T))$, for a general OBA $(A, C)$ and w.r.t. an arbitrary Banach algebra homomorphism $T: A \rightarrow B$, and we shall see that the set $A^{-1}+(C \cap \mathrm{N}(T))$ does, in general, not necessarily equal $\mathcal{W}_{T}$.

We shall begin this chapter with the definitions of upper Weyl and upper Browder elements relative to a fixed Banach algebra homomorphism. In Section 3.2 we point out a class of Banach algebra homomorphisms relative to which every Weyl element can in fact be decomposed as a sum of an invertible element and a positive element in the null space of the homomorphism. Subsequently, we present a general discussion on the basic (algebraic) properties of upper Weyl and upper Browder elements in an OBA. Results pertaining to the perturbation of upper Weyl (and upper Browder) elements by elements from a number of classes will be discussed in Section 3.4. In our final section, we examine regularities in connection with upper Weyl and upper Browder elements.

Throughout, the reader should keep in mind the homomorphisms $\pi$ : $\mathcal{L}(E) \rightarrow \mathcal{L}(E) / \mathcal{K}(E)$ and $\pi_{r}: \mathcal{L}^{r}(E) \rightarrow \mathcal{L}^{r}(E) / \mathcal{K}^{r}(E)$, in which case $\mathrm{N}(\pi)=$ 
$\mathcal{K}(E)$ and $\mathrm{N}\left(\pi_{r}\right)=\mathcal{K}^{r}(E)$.

\subsection{Definitions and examples}

In this section we introduce and discuss upper Weyl and upper Browder elements relative to a fixed Banach algebra homomorphism. These are special kinds of Weyl and Browder elements, respectively.

Definition 3.1.1 (Upper Weyl and upper Browder elements). Let $(A, C)$ be an $\mathrm{OBA}$ and $\mathrm{T}: A \rightarrow B$ be a Banach algebra homomorphism. An element $a \in A$ is called

- upper Weyl if there exist elements $b \in A^{-1}$ and $c \in C \cap \mathrm{N}(T)$ such that $a=b+c$,

- upper Browder if there exist commuting elements $b \in A^{-1}$ and $c \in C \cap$ $\mathrm{N}(T)$ such that $a=b+c$.

Evidently, Definition 3.1.1 brings into play the positivity theory in the abstract Fredholm theory.

Denote by $\mathcal{W}_{T}^{+}$and $\mathcal{B}_{T}^{+}$the sets of all upper Weyl and upper Browder elements of $A$ relative to $T$, respectively. As a starter, we describe the upper Weyl (upper Browder) elements in particular OBAs relative to certain Banach algebra homomorphisms.

The following fact is due to Alekhno.

Example 3.1.2. ([4], Theorem 3; this thesis Example 1.5.2(c)) Let E be a Banach lattice. Then $T \in \mathcal{W}_{\pi}^{+}$if and only if $T$ is Fredholm with index zero.

Example 3.1.3. Let $E$ be a Banach lattice. Then $\mathcal{W}_{\pi_{r}}=\mathcal{W}_{\pi_{r}}^{+}$; that is, $T \in \mathcal{W}_{\pi_{r}}^{+}$if and only if $T$ can be decomposed as a sum of an invertible operator in $\mathcal{L}^{r}(E)$ and a finite rank operator on $E$.

First we show that $\mathcal{K}^{r}(E) \subseteq$ span $\left(K \cap \mathcal{K}^{r}(E)\right)$, where $K:=\{T \in \mathcal{L}(E)$ : $\left.T E_{+} \subseteq E_{+}\right\}$. Let $S \in \mathcal{K}^{r}(E)$. Then there exist $U_{\mathbb{C}}, V_{\mathbb{C}} \in \mathcal{K}^{r}(E)$ such that $S=U_{\mathbb{C}}+i V_{\mathbb{C}}$. By $\left([8]\right.$, Theorem 1.3) the positive and negative parts of $U_{\mathbb{C}}$ and $V_{\mathbb{C}}$ are again $r$-compact, and hence

$$
S=U_{\mathbb{C}}^{+}-U_{\mathbb{C}}^{-}+i V_{\mathbb{C}}^{+}-i V_{\mathbb{C}}^{-} \in \operatorname{span}\left(K \cap \mathcal{K}^{r}(E)\right)
$$


Now, if $T \in \mathcal{W}_{\pi_{r}}$, then $T=R+S$ for some $R \in\left(\mathcal{L}^{r}(E)\right)^{-1}$ and $S \in$ $\mathcal{K}^{r}(E)$. Using the identity $\mathcal{K}^{r}(E)=$ span $\left(K \cap \mathcal{K}^{r}(E)\right)$ and a slight modification of ([4], Lemma 1) (or, more generally, Lemma 3.2.2 which we establish in the next section), it follows that $T \in\left(\mathcal{L}^{r}(E)\right)^{-1}+\left(K \cap \mathcal{K}^{r}(E)\right)=\mathcal{W}_{\pi_{r}}^{+}$, and thus $\mathcal{W}_{\pi_{r}}=\mathcal{W}_{\pi_{r}}^{+}$.

The last part of the example follows from ([9], Theorem 4.3) which gives $\mathcal{W}_{\pi_{r}}=\left(\mathcal{L}^{r}(E)\right)^{-1}+\mathcal{F}(E)$. (We mention that this fact is also clear from ([18], Lemma 5.3).

Presumably the identities $\mathcal{B}_{\pi}=\mathcal{B}_{\pi}^{+}$and $\mathcal{B}_{\pi_{r}}=\mathcal{B}_{\pi_{r}}^{+}$do not hold, but we are unable to provide counterexamples at present. That is, (as yet) we do not have an example of an operator $T$ such that $T \in \mathcal{B}_{\pi}\left(T \in \mathcal{B}_{\pi_{r}}\right)$, but $T \notin \mathcal{B}_{\pi}^{+}$ $\left(T \notin \mathcal{B}_{\pi_{r}}^{+}\right)$.

Modifying Example 1.5.3(b), we get the following. We remark that this example will play a significant role as a source of counterexamples in this thesis.

Example 3.1.4. Let $K_{1}$ and $K_{2}$ be compact Hausdorff spaces and $A:=C\left(K_{1}\right)$ and $B:=C\left(K_{2}\right)$ be the ordered Banach algebras of continuous complex-valued functions on $K_{1}$ and $K_{2}$, respectively. Consider the homomorphism $T: A \rightarrow B$ defined by $T f=f \circ \theta$, where $\theta: K_{2} \rightarrow K_{1}$ is a continuous map. Then $f \in$ $\mathcal{W}_{T}^{+}$if and only if its restriction to $\theta\left(K_{2}\right)$ has an invertible extension to $K_{1}$, say $g$, satisfying $f \geq g$.

Let $C:=\left\{f \in A: f(x) \in \mathbb{R}^{+}\right.$for all $\left.x \in K_{1}\right\}$. Suppose that $f \in \mathcal{W}_{T}^{+}$. Then there exist $g \in A^{-1}$ and $h \in C \cap \mathrm{N}(T)$ such that $f=g+h$. Since $h \in \mathrm{N}(T)$, we have that $h\left(\theta\left(K_{2}\right)\right)=\{0\}$, and hence

$$
f_{\mid \theta\left(K_{2}\right)}=g_{\mid \theta\left(K_{2}\right)}+h_{\mid \theta\left(K_{2}\right)}=g_{\mid \theta\left(K_{2}\right)} .
$$

Also, since $h \in C$, it follows that $f \geq g$. Consequently, if $f \in \mathcal{W}_{T}^{+}$, then $f_{\mid \theta\left(K_{2}\right)}$ has an invertible extension to $K_{1}$, namely $g$, satisfying $f \geq g$.

Conversely, suppose that $f_{\mid \theta\left(K_{2}\right)}$ has an invertible extension to $K_{1}$, say $g$, satisfying $f \geq g$. Observe that $T(f-g)=(f-g) \circ \theta=0$. Therefore, $f=g+(f-g)$, where $g \in A^{-1}$ and $f-g \in C \cap \mathrm{N}(T)$, so that $f \in \mathcal{W}_{T}^{+}$.

We call $T$ in Example 3.1.4 the homomorphism induced by composition with $\theta$.

Let $\left(A_{1}, C_{1}\right)$ and $\left(A_{2}, C_{2}\right)$ be OBAs and $T_{1}: A_{1} \rightarrow B_{1}$ and $T_{2}: A_{2} \rightarrow B_{2}$ be Banach algebra homomorphisms. Consider the $\mathrm{OBA}(A, C)$ and Banach 
algebra $B$, where $A:=A_{1} \oplus A_{2}, C:=C_{1} \oplus C_{2}$ and $B:=B_{1} \oplus B_{2}$. One can easily verify that $T: A \rightarrow B$ defined by $T\left(a_{1}, a_{2}\right)=\left(T_{1} a_{1}, T_{2} a_{2}\right)$ is a Banach algebra homomorphism. Moreover, it follows that $\left(a_{1}, a_{2}\right) \in \mathcal{W}_{T}^{+}$if and only if $a_{1} \in \mathcal{W}_{T_{1}}^{+}$and $a_{2} \in \mathcal{W}_{T_{2}}^{+}$and that $\left(a_{1}, a_{2}\right) \in \mathcal{B}_{T}^{+}$if and only if $a_{1} \in \mathcal{B}_{T_{1}}^{+}$and $a_{2} \in \mathcal{B}_{T_{2}}^{+}$.

Proposition 3.1.5. Let $(A, C)$ be an $O B A$ and $T: A \rightarrow B$ be a Banach algebra homomorphism. Then the following set of inclusions holds in general:

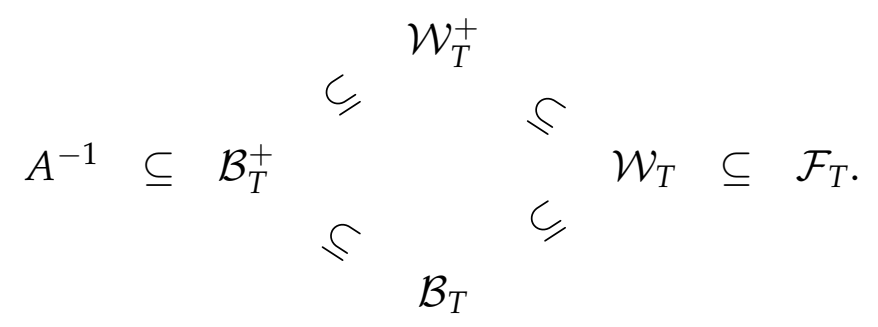

Equalities are obtained whenever $T$ is spectrum preserving.

Proof. By definition the inclusions $\mathcal{B}_{T}^{+} \subseteq \mathcal{B}_{T}$ and $\mathcal{B}_{T}^{+} \subseteq \mathcal{W}_{T}^{+} \subseteq \mathcal{W}_{T}$ hold. It remains to recall Proposition 1.5.4 to finish the proof.

Suppose now that $T$ is spectrum preserving. Then $\sigma(T a)=\sigma(a)$ for all $a \in A$, and hence $A^{-1}=\mathcal{F}_{T}$. The result follows from the first part of the proposition.

The following examples illustrate that the above inclusions can be strict.

Example 3.1.6. Consider the $O B A\left(\mathbb{C}^{2}, \mathbb{R}^{2}\right)$ and the homomorphism $T: \mathbb{C}^{2} \rightarrow \mathbb{C}$ defined by $T(z, w)=z$. Then $\left(\mathbb{C}^{2}\right)^{-1} \subsetneq \mathcal{B}_{T}^{+}$.

It is easy to see that $\mathrm{N}(T)=\{(0, \lambda): \lambda \in \mathbb{C}\}$. Consider the element $(1,0) \in \mathbb{C}^{2}$. Then $(1,0)=(1,-1)+(0,1)$, where $(1,-1) \in\left(\mathbb{C}^{2}\right)^{-1}$ and $(0,1) \in \mathbb{R}^{2} \cap \mathrm{N}(T)$. Hence, $(1,0) \in \mathcal{B}_{T}^{+}$, but $(1,0) \notin\left(\mathbb{C}^{2}\right)^{-1}$.

Example 3.1.7. Consider the Banach lattice $l^{2}:=l^{2}(\mathbb{C})$ of all sequences $\left(x_{n}\right)_{n \in \mathbb{N}}$ of complex numbers such that $\sum_{n=1}^{\infty}\left|x_{n}\right|^{2}<\infty$ and the canonical homomorphism $\phi: \mathfrak{L}\left(l^{2} \oplus l^{2}\right) \rightarrow \mathfrak{L}\left(l^{2} \oplus l^{2}\right) / \mathcal{K}\left(l^{2} \oplus l^{2}\right)$. Then $\mathcal{B}_{\phi}^{+} \subsetneq \mathcal{W}_{\phi}^{+}$.

Define $U, V: l^{2} \rightarrow l^{2}$ by $U\left(x_{1}, x_{2}, \ldots\right)=\left(0, x_{1}, x_{2}, \ldots\right)$ and $V\left(x_{1}, x_{2}, \ldots\right)=$

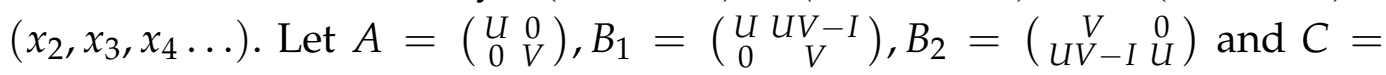
$\left(\begin{array}{cc}0 & I-U V \\ 0 & 0\end{array}\right)$. By using some information on $U$ and $V$ as discussed in ([21], pp.172-173), we have that $A=B_{1}+C$, where $B_{1} B_{2}=I=B_{2} B_{1}$ and $C$ is a finite rank operator. Since $C \in \mathrm{N}(\phi)$ is a positive operator, we have that 
$A$ is upper Weyl. From ([21], Example 4.3) $A$ is not Browder, and hence not upper Browder by Proposition 3.1.5.

Example 3.1.8. Consider the $O B A(C(K), C)$, where $K=[0,1]$ and $C:=\{h \in$ $C(K): h(x) \in \mathbb{R}^{+}$for all $\left.x \in K\right\}$, and let $T: C(K) \rightarrow C(K)$ be the homomorphism induced by composition with the unit function 1 on $K$. Then $\mathcal{W}_{T}^{+} \subsetneq \mathcal{W}_{T}$.

We consider the function $f \in C(K)$ defined by $f(z)=z$ for all $z \in K$. Because $\mathbf{1}(K)=\{1\}$, we can extend $f_{\mid \mathbf{1}(K)}$ to the constant function $\mathbf{1}$ on $K$ which is invertible. By Example 1.5.3(b), we have that $f \in \mathcal{W}_{T}$.

Suppose that $f$ is also upper Weyl. By Example 3.1.4, $f$ restricted to $\mathbf{1}(K)=\{1\}$ has an invertible extension to $K$, say $g$, satisfying $g \leq f$. Note that $g(K) \subseteq \mathbb{R}$. By the continuity of $g$ and the facts that $g(1)=1$ and $g(0) \leq$ $f(0)=0$, we have from the intermediate value theorem that there exists $x \in K$ such that $g(x)=0$. This yields a contradiction to the invertibility of $g$; hence $f \notin \mathcal{W}_{T}^{+}$.

Using the fact that $C(K)$ is a commutative OBA, Example 3.1.8 remains true if $\mathcal{W}_{T}^{+}$and $\mathcal{W}_{T}$ are replaced by $\mathcal{B}_{T}^{+}$and $\mathcal{B}_{T}$, respectively.

Example 3.1.9. An example of a Fredholm element which is not Weyl can be found in ([4], Example 19), while ([21], Example 4.3) gives an example of a Weyl element which is not Browder.

The homomorphism $T$ (or a variation thereof) defined in Example 3.1.8 will be encountered in a number of examples in the remainder of this thesis.

\subsection{Examining the equation $\mathcal{W}_{\mathrm{T}}=\mathcal{W}_{\mathrm{T}}^{+}$}

A natural task, in view of ([4], Theorem 3), is to find, in general, classes of Banach algebra homomorphisms $T$ that satisfy $\mathcal{W}_{T}=\mathcal{W}_{T}^{+}$. Recall Example 3.1.8 which indicates that the identity does not hold in general.

Before we present our findings in the more general setting, we first review the operator case. All through this section, let $E$ denote a Banach lattice and $K=\left\{T \in \mathcal{L}(E): T E_{+} \subseteq E_{+}\right\}$. Theorem 3 in [4], a result due to Alekhno, tells us that the identity $\mathcal{W}_{\pi}=\mathcal{L}(E)^{-1}+(K \cap \mathrm{N}(\pi))=\mathcal{W}_{\pi}^{+}$holds.

Some remarks: It should be noted that the first tool to Alekhno's advantage was the fact that an element of $\mathcal{W}_{\pi}$ can be decomposed as a sum of 
an invertible operator and a finite rank operator (see Example 1.5.2(c)), implying $\mathcal{W}_{\pi}=\mathcal{W}_{\phi}$, where $\phi: \mathcal{L}(E) \rightarrow \mathcal{L}(E) / \overline{\mathcal{F}(E)}$ (and as understood $\pi: \mathcal{L}(E) \rightarrow \mathcal{L}(E) / \mathcal{K}(E))$. This is a crucial starting point as it is the special properties of finite rank operators, not necessarily valid for compact operators, on which Alekhno's proof relies. Secondly, the fact that every (real) finite rank operator's positive and negative parts exist ([7], Theorem 16.8) (this particular fact is for instance not in general true for compact operators (U. Krengel, see [7], Example 16.6)) was yet another important ingredient. (It is interesting to note that this statement is true despite the fact that $\mathcal{L}(E)$ is not a Riesz space in general.) Consequently, with the help of ([4], Lemma 2), he establishes the inclusion $\mathcal{F}(E) \subseteq \operatorname{span}(K \cap \overline{\mathcal{F}(E)})$ : ([4], Lemma 2) states that the positive and negative parts of a finite rank operator are elements of $K \cap \overline{\mathcal{F}(E)}$. Finally, it remains to recall ([4], Lemma 1) to finish his proof. Consequently,

$$
\mathcal{W}_{\pi}^{+}=\mathcal{W}_{\pi}=\mathcal{W}_{\phi}=\mathcal{W}_{\phi}^{+}
$$

In our development leading up to an OBA-version of Alekhno's result ([4], Theorem 3), we first establish the following generalization of ([4], Lemma 1) for arbitrary Banach algebra elements. The same proof applies, but we include the (more detailed) proof in the interest of completeness.

Lemma 3.2.2. Let $A$ be a Banach algebra and $I$ be an inessential ideal of $A$. If $a \in A^{-1}, b \in I$ and $\lambda \in \mathbb{C}$, then there exist $a_{1} \in A^{-1}$ and $\mu \geq 0$ such that $a+\lambda b=a_{1}+\mu b$.

Proof. Let $a \in A^{-1}, b \in I$ and $\lambda \in \mathbb{C}$. If $\lambda \geq 0$, then the result holds with $a_{1}=a$ and $\mu=\lambda$. Hence, suppose that $\lambda \in \mathbb{C} \backslash\{\lambda \in \mathbb{C}: \lambda \geq 0\}$. Seeing that $I$ is an ideal, we have that $a^{-1} b \in I$. Since $I$ is inessential, $\sigma\left(a^{-1} b\right)$ must have at most 0 as an accumulation point.

Suppose that $\frac{1}{\mu-\lambda} \in \sigma\left(a^{-1} b\right)$ for all $\mu \geq 0$. Then $\frac{1}{\frac{1}{n}-\lambda} \in \sigma\left(a^{-1} b\right)$ for all $n \in \mathbb{N}$, and hence $-\frac{1}{\lambda}=\lim _{n \rightarrow \infty} \frac{1}{\frac{1}{n}-\lambda} \in \sigma\left(a^{-1} b\right)$ by the closedness of the spectrum, so that $-\frac{1}{\lambda} \in \operatorname{acc} \sigma\left(a^{-1} b\right)$. But this contradicts the fact that $I$ is inessential; hence there exists $\mu \geq 0$ such that $\frac{1}{\mu-\lambda} \notin \sigma\left(a^{-1} b\right)$.

For such $\mu$, we have that $(\mu-\lambda)\left[\frac{1}{\mu-\lambda} \mathbf{1}-a^{-1} b\right] \in A^{-1}$; that is, $\mathbf{1}-(\mu-$ $\lambda) a^{-1} b \in A^{-1}$. Consequently,

$$
a+\lambda b=a-(\mu-\lambda) b+\mu b=a\left[\mathbf{1}-(\mu-\lambda) a^{-1} b\right]+\mu b .
$$


Let $a_{1}=a\left[\mathbf{1}-(\mu-\lambda) a^{-1} b\right]$. The proof is completed as $a_{1} \in A^{-1}$.

As a consequence of Lemma 3.2.2, we have the following result which will be used a number of times in the sequel.

Corollary 3.2.3. Let $A$ be a Banach algebra and $I$ be an inessential ideal of $A$. If $a=b+\lambda_{1} c_{1}+\lambda_{2} c_{2}+\cdots+\lambda_{n} c_{n}$ for some scalars $\lambda_{1}, \ldots, \lambda_{n} \in \mathbb{C}, b \in A^{-1}$ and $c_{1}, \ldots, c_{n} \in I$, then a has the form

$$
a=b_{n}+\mu_{1} c_{1}+\mu_{2} c_{2}+\cdots+\mu_{n} c_{n}
$$

where $\mu_{1}, \ldots, \mu_{n} \geq 0$ and $b_{n} \in A^{-1}$.

Proof. Since $b \in A^{-1}, c_{1} \in I$ and $\lambda_{1} \in \mathbb{C}$, we have from Lemma 3.2.2 the existence of a $b_{1} \in A^{-1}$ and $\mu_{1} \geq 0$ such that

$$
\begin{aligned}
a & =\left(b+\lambda_{1} c_{1}\right)+\lambda_{2} c_{2}+\lambda_{3} c_{3}+\cdots+\lambda_{n} c_{n} \\
& =b_{1}+\mu_{1} c_{1}+\lambda_{2} c_{2}+\lambda_{3} c_{3}+\cdots+\lambda_{n} c_{n} \\
& =\mu_{1} c_{1}+\left(b_{1}+\lambda_{2} c_{2}\right)+\lambda_{3} c_{3}+\cdots+\lambda_{n} c_{n} .
\end{aligned}
$$

Now, since $b_{1} \in A^{-1}, c_{2} \in I$ and $\lambda_{2} \in \mathbb{C}$, we again apply Lemma 3.2.2 to find an element $b_{2} \in A^{-1}$ and a positive scalar $\mu_{2} \geq 0$ such that

$$
\begin{aligned}
a & =\mu_{1} c_{1}+\left(b_{1}+\lambda_{2} c_{2}\right)+\lambda_{3} c_{3}+\cdots+\lambda_{n} c_{n} \\
& =\mu_{1} c_{1}+\left(b_{2}+\mu_{2} c_{2}\right)+\lambda_{3} c_{3}+\cdots+\lambda_{n} c_{n} \\
& =\mu_{1} c_{1}+\mu_{2} c_{2}+\left(b_{2}+\lambda_{3} c_{3}\right)+\cdots+\lambda_{n} c_{n} .
\end{aligned}
$$

If we continue with this procedure and the use of Lemma 3.2.2, we obtain after $n$ steps $b_{n} \in A^{-1}$ and $\mu_{n} \geq 0$ such that

$$
a=\mu_{1} c_{1}+\mu_{2} c_{2}+\cdots+\mu_{n} c_{n}+b_{n}
$$

This completes our proof.

Considering the canonical homomorphism $\pi: A \rightarrow A / \bar{I}$, where $I$ is an inessential ideal of an OBA $(A, C)$, we remark that if $I \subseteq \operatorname{span}(C \cap \bar{I})$, then $\mathcal{W}_{\pi}=\mathcal{W}_{\pi}^{+}$. This remark can be seen from ([18], Lemma 5.3), which gives $\mathcal{W}_{\pi}=A^{-1}+I$, and by applying Corollary 3.2.3. This fact indicates a generalization of the second part of (3.2.1).

In the rest of the section we consider more general Banach algebra homomorphisms. 
Observe that the inclusion $\mathcal{F}(E) \subseteq$ span $(K \cap \overline{\mathcal{F}(E)})$ (in this particular form) does not enable us to get a more precise assumption on the Banach algebra homomorphism $\phi$, seeing that $\mathcal{F}(E)$ has no expression in terms of $\phi$. Evidently, only by taking closures are we able to get a grip on $\phi$ as can be seen in the following remark.

Remark 3.2.4. Let $E$ be a Banach lattice and consider the canonical homomorphism $\phi: \mathcal{L}(E) \rightarrow \mathcal{L}(E) / \overline{\mathcal{F}(E)}$. Then

$$
\overline{\mathrm{N}(\phi)}=\mathrm{N}(\phi)=\overline{\mathcal{F}(E)}=\overline{\operatorname{span}(K \cap \overline{\mathcal{F}(E)})}=\overline{\operatorname{span}(K \cap \mathrm{N}(\phi))} .
$$

Let $(A, C)$ be an arbitrary OBA. Motivated by equations (3.2.1) and (3.2.5), we are led to study general Banach algebra homomorphisms $T: A \rightarrow B$ satisfying the Riesz property and the condition $\overline{\mathrm{N}(T)}=\overline{\operatorname{span}(C \cap \mathrm{N}(T))}$.

Under the assumption that $T$ satisfies the Riesz property we observe that there is inclusion $A^{-1}+\overline{\operatorname{span}(C \cap \mathrm{N}(T))} \subseteq \overline{\mathcal{W}_{T}^{+}}$:

If $a \in A^{-1}+\overline{\operatorname{span}(C \cap \mathrm{N}(T))}$, then there exist elements $b \in A^{-1}$ and $c \in$ $\overline{\operatorname{span}(C \cap \mathrm{N}(T))}$ satisfying $a=b+c$. Consequently, we can find a sequence $\left(c_{n}\right)$ in $\operatorname{span}(C \cap \mathrm{N}(T))$ such that

$$
a=b+\lim _{n \rightarrow \infty} c_{n}=\lim _{n \rightarrow \infty}\left(b+\lambda_{1 n} c_{1 n}+\cdots+\lambda_{m n} c_{m n}\right)
$$

for some scalars $\lambda_{1 n}, \ldots \lambda_{m n} \in \mathbb{C}$ and $c_{1 n}, \ldots, c_{m n} \in C \cap \mathrm{N}(T)$. In view of Corollary 3.2.3, the statement $a \in \overline{\mathcal{W}_{T}^{+}}$holds.

If, in addition, $T$ satisfies the condition $\overline{\mathrm{N}(T)}=\overline{\operatorname{span}(C \cap \mathrm{N}(T))}$, it then follows that

$$
\begin{aligned}
\overline{\mathcal{W}_{T}}=\overline{A^{-1}+\mathrm{N}(T)} & \subseteq \overline{A^{-1}+\overline{\mathrm{N}(T)}} \\
& =\overline{A^{-1}+\overline{\operatorname{span}(C \cap \mathrm{N}(T))}} \\
& \subseteq \overline{\overline{\mathcal{W}_{T}^{+}}}=\overline{\mathcal{W}_{T}^{+}}
\end{aligned}
$$

that is, $\overline{\mathcal{W}_{T}}=\overline{\mathcal{W}_{T}^{+}}$, in consideration of Proposition 3.1.5.

Therefore, we have proven that if $T: A \rightarrow B$ has the Riesz property and satisfies the condition $\overline{\mathrm{N}(T)}=\overline{\operatorname{span}(\mathrm{C} \cap \mathrm{N}(T))}$, then $\overline{\mathcal{W}_{T}}=\overline{\mathcal{W}_{T}^{+}}$. However, this is not a new fact. As a matter of fact, the equality $\overline{\mathcal{W}_{T}}=\overline{\mathcal{W}_{T}^{+}}$is true even if we had only assumed that $T$ has the Riesz property: ([29], Corollary 3.8) gives $\overline{\mathcal{W}_{T}}=\overline{A^{-1}}$, and hence $\overline{\mathcal{W}_{T}}=\overline{\mathcal{W}_{T}^{+}}$in light of Proposition 3.1.5.

Strengthening the assumption $\overline{\mathrm{N}(T)}=\overline{\operatorname{span}(\mathrm{C} \cap \mathrm{N}(T))}$ to the condition $\mathrm{N}(T)=$ span $(C \cap \mathrm{N}(T))$ leads to the following fact (Theorem 3.2.6(a)) 
which is the main result in this section. It describes in general relative to which Banach algebra homomorphisms every Weyl element is a sum of an invertible element and a positive null space element. We point out that a glance at the discussion above reveals that, under the stronger assumption $\mathrm{N}(T)=\operatorname{span}(C \cap \mathrm{N}(T))$ (in which case no limits are used), the equality $\mathcal{W}_{T}=\mathcal{W}_{T}^{+}$is obtained. For the sake of completeness we provide the proof.

Theorem 3.2.6. Let $(A, C)$ be an $O B A$ and $T: A \rightarrow B$ be a Banach algebra homomorphism. Each of the following assumptions ensures that $\mathcal{W}_{T}=\mathcal{W}_{T}^{+}$:

(a) $T$ has the Riesz property and satisfies $\mathrm{N}(T)=\operatorname{span}(C \cap \mathrm{N}(T))$,

(b) $T$ is spectral radius preserving.

Proof. For the non-trivial inclusion assume that $a \in \mathcal{W}_{T}$. Then there exist $b \in A^{-1}$ and $c \in \mathrm{N}(T)$ such that $a=b+c$.

(a) Since $c \in \mathrm{N}(T)$, we have from assumption the existence of scalars $\lambda_{1}, \lambda_{2}, \ldots, \lambda_{n} \in \mathbb{C}$ and $c_{1}, c_{2}, \ldots, c_{n} \in C \cap \mathrm{N}(T)$ satisfying $c=\lambda_{1} c_{1}+\cdots+$ $\lambda_{n} c_{n}$, and hence $a=b+\lambda_{1} c_{1}+\cdots+\lambda_{n} c_{n}$. Using Corollary 3.2.3 we can find $b_{n} \in A^{-1}$ and $\mu_{1}, \mu_{2}, \ldots, \mu_{n} \geq 0$ such that

$$
a=b_{n}+\mu_{1} c_{1}+\mu_{2} c_{2}+\cdots+\mu_{n} c_{n}
$$

Let $c^{\prime}:=\mu_{1} c_{1}+\mu_{2} c_{2}+\cdots+\mu_{n} c_{n}$. Then $a=b_{n}+c^{\prime}$, where $b_{n} \in A^{-1}$ and $c^{\prime} \in C \cap \mathrm{N}(T)$. Hence $a \in \mathcal{W}_{T}^{+}$.

(b) From Lemma 1.2.7 we have that $c \in \operatorname{Rad}(A)$, and hence $a=b+c \in$ $A^{-1}$ by Theorem 1.1.3. Using Proposition 3.1.5 it follows that $A^{-1}=\mathcal{W}_{T}$, so that $\mathcal{W}_{T}=\mathcal{W}_{T}^{+}$.

It is worth mentioning that Theorem 3.2.6(a) is a generalization of Example 3.1.3, where the homomorphism $\pi_{r}$ has the Riesz property and the identity $\mathrm{N}\left(\pi_{r}\right)=$ span $\left(K \cap \mathrm{N}\left(\pi_{r}\right)\right)$ holds. It does not generalize Example 3.1.2 as the identity $\mathrm{N}(\pi)=$ span $(K \cap \mathrm{N}(\pi))$ does not hold in general (see Example 3.2.8). We point out that the latter identity, together with the equality $\mathrm{N}(\phi)=$ span $(K \cap \mathrm{N}(\phi)$, hold whenever the Banach lattice $E$ is either $A L$ or $A M$ (recall Lemma 1.6.9).

Remark 3.2.7. Observe from the proof of Theorem 3.2.6(b) that

$$
A^{-1}=\mathcal{B}_{T}^{+}=\mathcal{B}_{T}=\mathcal{W}_{T}^{+}=\mathcal{W}_{T}
$$

whenever the Banach algebra homomorphism $\mathrm{T}$ is spectral radius preserving. 
Let us mention that without the assumption about the Riesz property Theorem 3.2.6(a) is not in general true (see Example 3.1.8). Furthermore, as suspected (or even expected), in view of (3.2.5), the span-condition in Theorem 3.2.6(a) is not necessary for the validity of the identity $\mathcal{W}_{T}^{+}=\mathcal{W}_{T}$.

Example 3.2.8. Consider the (real) Banach lattice $E_{\mathbb{R}}:=C[0,1] \oplus l^{2}(\mathbb{R})$ and the canonical homomorphism $\pi: \mathcal{L}(E) \rightarrow \mathcal{L}(E) / \mathcal{K}(E)$, where $E=E_{\mathbb{R}} \oplus i E_{\mathbb{R}}$ is the complexification of $E_{\mathbb{R}}$. Then $\mathcal{W}_{\pi}=\mathcal{W}_{\pi}^{+}$, but $\operatorname{span}(K \cap \mathrm{N}(\pi)) \subsetneq \mathrm{N}(\pi)$, where $K=\left\{T \in \mathcal{L}(E): T E_{+} \subseteq E_{+}\right\}$.

It is well-known that $\pi$ satisfies the Riesz property and that $\mathcal{W}_{\pi}=\mathcal{W}_{\pi}^{+}$ by Example 3.1.2. We now show that $\mathrm{N}(\pi) \neq \operatorname{span}(K \cap \mathrm{N}(\pi))$.

Since $C[0,1]$ is not a $K B$-space (as remarked earlier in this thesis, just below Lemma 1.9.5), we have that $E_{\mathbb{R}}$ is not a $K B$-space. We also have from ([35], Examples, p.129) and ([7], Theorem 19.6) that $E_{\mathbb{R}}$ is not an $A M$-space. It then follows from Theorem 1.6.8 that there exists a compact operator $T: E_{\mathbb{R}} \rightarrow E_{\mathbb{R}}$ which is not regular. Hence $T_{\mathbb{C}} \in \mathcal{L}(E)$ is compact; that is $T_{\mathbb{C}} \in \mathrm{N}(\pi)$, and not regular. This gives $T_{\mathbb{C}} \notin \operatorname{span}(K \cap \mathrm{N}(\pi))$, and hence the result follows.

Even though Example 3.2.8 illustrates that the span-condition in Theorem 3.2.6(a) is not necessary in general, (in the approach we used) to establish the identity $\mathcal{W}_{T}^{+}=\mathcal{W}_{T}$ we were not able to survive without this condition. Note that the homomorphism $T$ in Example 3.1.8 (which gives $\mathcal{W}_{T}^{+} \subsetneq \mathcal{W}_{T}$ ) satisfies the span-condition, but does not have the Riesz property. Therefore, the importance of the span-condition (next to the assumption about the Riesz property) in Theorem 3.2.6(a) remains a mystery until we can answer the following question: Is there an example of a homomorphism $T$ which satisfies the Riesz property such that $\mathcal{W}_{T}^{+} \subsetneq \mathcal{W}_{T}$ ? At present we do not have such an example.

Corollary 3.2.9. Let $(A, C)$ be an $O B A$ and $T: A \rightarrow B$ be a Banach algebra homomorphism. Under each of the following assumptions we have that $\mathcal{B}_{T}=\mathcal{B}_{T}^{+}$:

(a) A is commutative, $T$ has the Riesz property and satisfies the identity $\mathrm{N}(T)=$ $\operatorname{span}(C \cap \mathrm{N}(T))$,

(b) $T$ is spectral radius preserving.

Proof. The result is clear from Theorem 3.2.6 and Remark 3.2.7. 
We conclude this section with some examples of Banach algebra homomorphisms $T$ for which the equality $\mathcal{W}_{T}=\mathcal{W}_{T}^{+}$(or $\mathcal{B}_{T}=\mathcal{B}_{T}^{+}$in the cases where the domain of $T$ is commutative) holds. We point out that, since $\mathrm{N}(T)$ is a vector space, the inclusion $\operatorname{span}(C \cap \mathrm{N}(T)) \subseteq \mathrm{N}(T)$ is always true. Also, in the case of finite-dimensional OBAs, $\mathrm{N}(T)$ is an inessential ideal (as the spectrum of each OBA element is finite), and therefore $T$ becomes a homomorphism which satisfies the Riesz property.

Example 3.2.10. Let $n \in \mathbb{N}$ and consider the homomorphism $T: \mathbb{C}^{n} \rightarrow \mathbb{C}$ defined by $T\left(x_{1}, x_{2}, \ldots, x_{n}\right)=x_{1}$. Then $\mathcal{W}_{T}=\mathcal{W}_{T}^{+}$, and hence

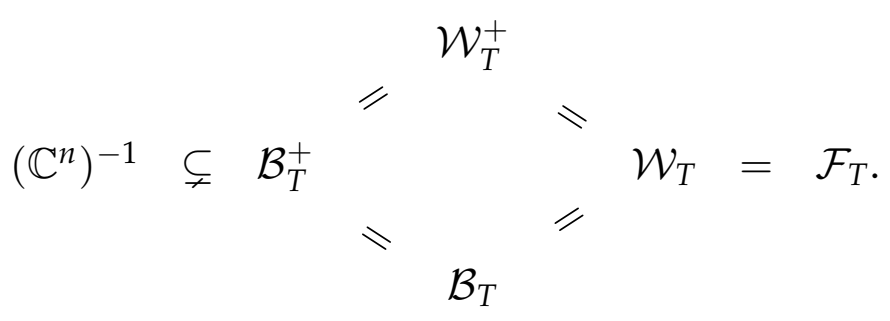

Let $C:=\left(\mathbb{R}^{n}\right)^{+}$. Obviously, the element $(1,0, \ldots, 0)$ is not invertible in $\mathbb{C}^{n}$. Nonetheless,

$$
(1,0, \ldots, 0)=(1,-1, \ldots,-1)+(0,1, \ldots, 1) \in\left(\mathbb{C}^{n}\right)^{-1}+(C \cap N(T))=\mathcal{B}_{T}^{+}
$$

Hence $\left(\mathbb{C}^{n}\right)^{-1} \subsetneq \mathcal{B}_{T}^{+}$.

Denote by $e_{n}$ the $n$-tuple whose $n$th term is 1 and all other terms are zero. If $x \in \mathrm{N}(T)$, then there exist $x_{k}:=a_{k}+b_{k} i$, where $a_{k}, b_{k} \in \mathbb{R}$ for $2 \leq k \leq n$, such that

$$
x=\left(0, x_{2}, \ldots, x_{n}\right)=\left(0, a_{2}, \ldots, a_{n}\right)+i\left(0, b_{2}, \ldots, b_{n}\right) .
$$

For $t, s \in \mathbb{N}$, let $\left\{a_{k_{2}}, \ldots, a_{k_{t}}\right\}=\left\{a_{2}, \ldots, a_{n}\right\} \cap \mathbb{R}^{+}$and take $\left\{b_{l_{2}}, \ldots, b_{l_{s}}\right\}$ $=\left\{b_{2}, \ldots, b_{n}\right\} \cap \mathbb{R}^{+}$. Then the elements $a_{k_{t+1}}, \ldots, a_{k_{n}}, b_{l_{s+1}}, \ldots b_{l_{n}}$ are the negative real numbers in $\left\{a_{2}, \ldots a_{n}, b_{2}, \ldots, b_{n}\right\}$ and

$$
x=\sum_{j=2}^{t} a_{k_{j}} e_{k_{j}}+(-1) \sum_{j=t+1}^{n}\left(-a_{k_{j}}\right) e_{k_{j}}+i \sum_{j=2}^{s} b_{l_{j}} e_{l_{j}}+(-i) \sum_{j=s+1}^{n}\left(-b_{l_{j}}\right) e_{l_{j}} .
$$

We have shown that $x$ can be represented as a linear combination of $n$-tuples in $C \cap \mathrm{N}(T)$; that is $x \in \operatorname{span}(C \cap \mathrm{N}(T))$. Therefore $\mathrm{N}(T)=\operatorname{span}(C \cap \mathrm{N}(T))$ and by Theorem 3.2.6(a) we have that $\mathcal{W}_{T}=\mathcal{W}_{T}^{+}$. 
We mention that the set of Weyl elements (or upper Weyl elements) relative to $T$ is given by

$$
\mathcal{W}_{T}=\left\{\left(x_{1}, x_{2}, \ldots, x_{n}\right) \in \mathbb{C}^{n}: x_{1} \neq 0 \text { and } x_{2}, \ldots, x_{n} \in \mathbb{C}\right\}=\mathcal{W}_{T}^{+}
$$

A simple verification shows that this is precisely the set of Fredholm elements of $\mathbb{C}^{n}$ relative to $T$. Hence, by using the fact that $\mathbb{C}^{n}$ is commutative, the desired inclusion scheme follows.

Example 3.2.11. Consider the homomorphism $T: l^{\infty}(\mathbb{C}) \rightarrow l^{\infty}(\mathbb{C})$ defined by $T\left(x_{1}, x_{2}, \ldots\right)=\left(x_{2}, x_{3}, \ldots\right)$. Then $\mathcal{W}_{T}=\mathcal{W}_{T}^{+}$, and hence

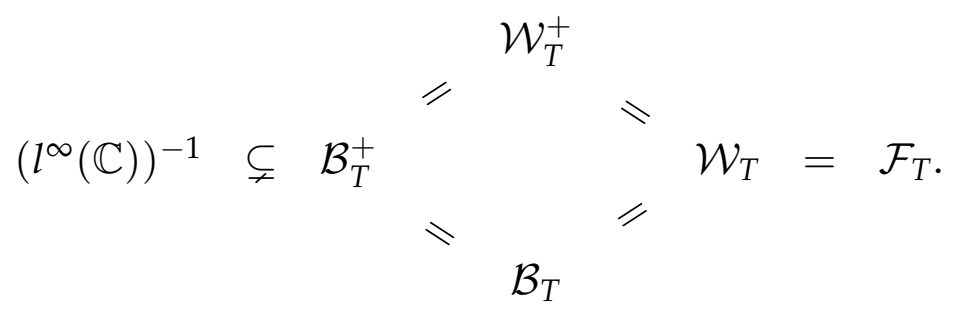

It is easy to see that $\mathrm{N}(T)=\left\{\left(x_{1}, 0,0, \ldots\right): x_{1} \in \mathbb{C}\right\}$. Since the spectrum of each element in $\mathrm{N}(T)$ is finite, we have that $\mathrm{N}(T)$ is inessential, and hence $T$ satisfies the Riesz property.

Take $a \in \mathrm{N}(T)$. Then there exists $z:=x+y i$, where $x, y \in \mathbb{R}$, such that $a=(z, 0,0, \ldots)=(x, 0,0, \ldots)+i(y, 0,0, \ldots)$. Depending on the signs of $x$ and $y$, a straightforward argument shows that $a$ can be represented as a linear combination of elements in $l^{\infty}\left(\mathbb{R}^{+}\right) \cap \mathrm{N}(T)$. Hence $a \in \operatorname{span}\left(l^{\infty}\left(\mathbb{R}^{+}\right) \cap\right.$ $\mathrm{N}(T))$, so that the identity $\mathrm{N}(T)=\operatorname{span}\left(l^{\infty}\left(\mathbb{R}^{+}\right) \cap \mathrm{N}(T)\right)$ holds. From Theorem 3.2.6(a) it follows that $\mathcal{W}_{T}=\mathcal{W}_{T}^{+}$.

We mention that the set of Weyl elements (or upper Weyl elements) relative to $T$ is given by

$\left\{\left(x_{1}, x_{2}, x_{3}, \ldots\right) \in l^{\infty}(\mathbb{C}): x_{1} \in \mathbb{C}\right.$ and $\left|x_{i}\right| \geq K$ for all $i \geq 2$ and some $\left.K>0\right\}$

This is precisely the set of Fredholm elements of $l^{\infty}(\mathbb{C})$ relative to $T$. Hence, by using the fact that $l^{\infty}(\mathbb{C})$ is commutative, the result follows.

The following example will be useful in the sequel. 
Example 3.2.12. Let $n \in \mathbb{N}$ and consider the homomorphism $T: M_{n}^{u}(\mathbb{C}) \rightarrow \mathbb{C}$ defined by

$$
T\left[\begin{array}{ccccc}
x_{11} & x_{12} & x_{13} & \cdots & x_{1 n} \\
0 & x_{22} & x_{23} & \cdots & x_{2 n} \\
0 & 0 & x_{33} & \cdots & x_{3 n} \\
\vdots & \vdots & \vdots & \ddots & \vdots \\
0 & 0 & 0 & \cdots & x_{n n}
\end{array}\right]=x_{11}
$$

Then $\mathcal{W}_{T}=\mathcal{W}_{T}^{+}$. Moreover,

$$
\begin{aligned}
& \mathcal{W}_{T}^{+}
\end{aligned}
$$

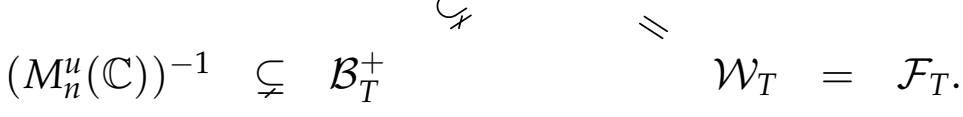

$$
\begin{aligned}
& £ \mathcal{B}_{T}
\end{aligned}
$$

Observe that $\mathrm{N}(T)$ consists of all upper triangular matrices with entry in the first row and first column zero.

Let $C:=M_{n}^{u}\left(\mathbb{R}^{+}\right)$. For the non-trivial inclusion suppose that $A \in \mathrm{N}(T)$. Since every element of $\mathrm{N}(T)$ is a matrix with at most $\frac{(n+2)(n-1)}{2}$ non-zero entries, we can force $A$, depending on the signs of the real and imaginary parts of the entries in $A$, to be written as a linear combination of at most $(n+$ $2)(n-1)$ elements (where each element is a matrix consisting of only one non-zero entry) in $C \cap \mathrm{N}(T)$. Hence $A \in \operatorname{span}(C \cap \mathrm{N}(T))$, so that $\mathrm{N}(T)=$ $\operatorname{span}(C \cap \mathrm{N}(T))$. By Theorem 3.2.6(a), we have that $\mathcal{W}_{T}=\mathcal{W}_{T}^{+}$.

Here, the Weyl elements (or upper Weyl elements) relative to $T$ are given by the set of all upper triangular matrices with entry in the first row and first column non-zero. An easy argument shows that this is precisely the set of Fredholm elements of $M_{n}^{u}(\mathbb{C})$ relative to $T$. Hence, by also using the fact that every square matrix is generalized Drazin invertible and Proposition 1.5.4, we have that $\mathcal{B}_{T}=\mathcal{W}_{T}=\mathcal{W}_{T}^{+}=\mathcal{F}_{T}$.

Obviously, the element

$$
n-1 \text { rows }\left\{\left(\begin{array}{ccccc}
1 & 0 & 0 & \cdots & 0 \\
0 & 0 & 0 & \cdots & 0 \\
0 & 0 & 0 & \cdots & 0 \\
\vdots & \vdots & \vdots & \ddots & \vdots \\
0 & 0 & 0 & \cdots & 0
\end{array}\right) \in M_{n}^{u}(\mathbb{C})\right.
$$


is not invertible. It is however upper Browder since it can be decomposed as the sum of

$$
\left(\begin{array}{ccccc}
1 & 0 & 0 & \cdots & 0 \\
0 & -1 & 0 & \cdots & 0 \\
0 & 0 & -1 & \cdots & 0 \\
\vdots & \vdots & \vdots & \ddots & \vdots \\
0 & 0 & 0 & \cdots & -1
\end{array}\right) \in M_{n}^{u}(\mathbb{C})^{-1} \text { and }\left(\begin{array}{ccccc}
0 & 0 & 0 & \cdots & 0 \\
0 & 1 & 0 & \cdots & 0 \\
0 & 0 & 1 & \cdots & 0 \\
\vdots & \vdots & \vdots & \ddots & \vdots \\
0 & 0 & 0 & \cdots & 1
\end{array}\right) \in C \cap \mathrm{N}(T)
$$

which commute. This gives $M_{n}^{u}(\mathbb{C})^{-1} \subsetneq \mathcal{B}_{T}^{+}$.

Consider the element

$$
n-1 \text { rows }\left\{\left(\begin{array}{ccccc}
1 & 1 & 0 & \cdots & 0 \\
0 & 0 & 0 & \cdots & 0 \\
0 & 0 & 0 & \cdots & 0 \\
\vdots & \vdots & \vdots & \ddots & \vdots \\
0 & 0 & 0 & \cdots & 0
\end{array}\right):=A\right.
$$

which is Browder. If $A=A_{1}+A_{2}$, where

$$
A_{1}:=\left(\begin{array}{ccccc}
1 & 1-x_{12} & -x_{13} & \cdots & -x_{1 n} \\
0 & -x_{22} & -x_{23} & \cdots & -x_{2 n} \\
0 & 0 & -x_{33} & \cdots & -x_{3 n} \\
\vdots & \vdots & \vdots & \ddots & \vdots \\
0 & 0 & 0 & \cdots & -x_{n n}
\end{array}\right)
$$

denotes an invertible element and

$$
A_{2}:=\left(\begin{array}{ccccc}
0 & x_{12} & x_{13} & \cdots & x_{1 n} \\
0 & x_{22} & x_{23} & \cdots & x_{2 n} \\
0 & 0 & x_{33} & \cdots & x_{3 n} \\
\vdots & \vdots & \vdots & \ddots & \vdots \\
0 & 0 & 0 & \cdots & x_{n n}
\end{array}\right)
$$

an arbitrary positive null space element, then $x_{i i} \neq 0$ for all $i \in\{2, \ldots, n\}$ and the entries in $A_{2}$ are non-negative real numbers. Now, $A_{1} A_{2}=A_{2} A_{1}$ implies that $x_{12}+x_{22}=0$, which is impossible as $x_{12} \geq 0$ and $x_{22}>0$. Hence $A$ is not upper Browder, so that $\mathcal{B}_{T}^{+} \subsetneq \mathcal{B}_{T}$.

Remark 3.2.13. Example 3.2.12 illustrates that the commutativity assumption on the domain of $T$ in Corollary 3.2.9(a) cannot in general be dropped. 


\subsection{Basic properties of upper Browder and upper Weyl elements}

This section summarizes some basic algebraic properties of upper Weyl and upper Browder elements. Throughout, we shall supply numerous examples indicating the necessity of the assumptions in our results.

We start our discussion with a number of corollaries derived from Lemma 3.2.2.

Corollary 3.3.1. Let $T: A \rightarrow B$ be a Banach algebra homomorphism which satisfies the Riesz property. If $a \in A^{-1}, b \in \mathrm{N}(T)$ and $\lambda \in \mathbb{C}$, then there exist $a_{1} \in A^{-1}$ and $\mu \geq 0$ such that $a+\lambda b=a_{1}+\mu b$.

Proof. The result follows from the fact that $\mathrm{N}(T)$ is an inessential ideal of $A$ and Lemma 3.2.2.

Corollary 3.3.1 then allows us to make the following two conclusions.

Corollary 3.3.2. Let $(A, C)$ be an $O B A$ and $T: A \rightarrow B$ be a Banach algebra homomorphism which satisfies the Riesz property. If $a \in A^{-1}, b \in C \cap \mathrm{N}(T)$ and $\lambda \in \mathbb{C}$, then $a+\lambda b \in \mathcal{W}_{T}^{+}$.

Proof. Let $a \in A^{-1}, b \in C \cap \mathrm{N}(T)$ and $\lambda \in \mathbb{C}$. By Corollary 3.3.1 there exist $a_{1} \in A^{-1}$ and $\mu \geq 0$ such that $a+\lambda b=a_{1}+\mu b$. Since $a_{1} \in A^{-1}$ and $\mu b \in C \cap \mathrm{N}(T)$, it follows that $a+\lambda b \in \mathcal{W}_{T}^{+}$.

Corollary 3.3.3. Let $(A, C)$ be an $O B A$ and $T: A \rightarrow B$ be a Banach algebra homomorphism which satisfies the Riesz property. If $a \in A^{-1}$ and $b \in C \cap \mathrm{N}(T)$ commute and $\lambda \in \mathbb{C}$, then $a+\lambda b \in \mathcal{B}_{T}^{+}$.

Proof. Let $a \in A^{-1}$ and $b \in C \cap \mathrm{N}(T) \cap \operatorname{Comm}(a)$ and $\lambda \in \mathbb{C}$. By Corollary 3.3.1 there exist $a_{1} \in A^{-1}$ and $\mu \geq 0$ such that $a+\lambda b=a_{1}+\mu b$. Using the fact that $a$ and $b$ commute, it follows that $a_{1}$ and $b$ also commute. Now, since $a_{1} \in A^{-1}$ and $\mu b \in C \cap \mathrm{N}(T)$ commute, we have that $a+\lambda b \in \mathcal{B}_{T}^{+}$.

It is a familiar fact that the sets of Weyl and Browder elements relative to an arbitrary Banach algebra homomorphism are closed under nonzero scalar multiplication. If $T$ denotes a Banach algebra homomorphism, 
we note that, though this is clearly the case for all non-negative real numbers, it does not seem obvious whether $\mathcal{W}_{T}^{+}$and $\mathcal{B}_{T}^{+}$are closed under nonzero scalar multiplication. Using Corollaries 3.3.2 and 3.3.3, our next result shows that $\mathcal{W}_{T}^{+}$and $\mathcal{B}_{T}^{+}$are closed under non-zero scalar multiplication whenever $T$ has the Riesz property. We point out here that this is an important observation and will be useful in proving Proposition 4.2.5.

Lemma 3.3.4. Let $(A, C)$ be an $O B A$ and $T: A \rightarrow B$ be a Banach algebra homomorphism which satisfies the Riesz property. If $0 \neq \lambda \in \mathbb{C}$, then $\lambda \mathcal{W}_{T}^{+} \subseteq \mathcal{W}_{T}^{+}$ and $\lambda \mathcal{B}_{T}^{+} \subseteq \mathcal{B}_{T}^{+}$.

Proof. Let $0 \neq \lambda \in \mathbb{C}$ and suppose that $a \in \mathcal{W}_{T}^{+}$. Then $a=b+c$, where $b \in A^{-1}$ and $c \in C \cap \mathrm{N}(T)$. Since $\lambda b \in A^{-1}$ and $c \in C \cap \mathrm{N}(T)$, we have from Corollary 3.3.2 that $\lambda a=\lambda b+\lambda c \in \mathcal{W}_{T}^{+}$. In a similar way, using Corollary 3.3.3, it can be shown that $a \in \mathcal{B}_{T}^{+}$implies $\lambda a \in \mathcal{B}_{T}^{+}$whenever $0 \neq \lambda \in \mathbb{C}$.

The next example shows that the assumption " $T$ has the Riesz property" in Lemma 3.3.4 cannot in general be omitted.

Example 3.3.5. Consider the $O B A(C(K), C)$, where $K=[0,1]$ and $C:=\{f \in$ $C(K): f(x) \in \mathbb{R}^{+}$for all $\left.x \in K\right\}$, and let $T: C(K) \rightarrow C(K)$ be the homomorphism induced by composition with the unit function 1 on $K$. If $h \in C(K)$ is defined by $h(z)=-z$ for all $z \in K$, then $h \in \mathcal{W}_{T}^{+}$, but $-h \notin \mathcal{W}_{T}^{+}$.

Since $\mathbf{1}(K)=\{1\}$, we can extend $h_{\mid \mathbf{1}(K)}$ to the constant function $\mathbf{- 1}$ on $K$ which is invertible and satisfies $h \geq \mathbf{- 1}$. From Example 3.1.4 we have that $h \in \mathcal{W}_{T}^{+}\left(=\mathcal{B}_{T}^{+}\right)$, but $-h$ is not upper Weyl (upper Browder) by Example 3.1.8. We note that, since $\mathrm{N}(T)=\{w \in C(K): w(1)=0\}$, $T$ does not satisfy the Riesz property.

Although our next result is of independent interest, we state it here for use in Theorem 3.3.8(c). It generalizes Lemma 3.3.4 to arbitrary invertible OBA elements which are linear combinations of positive elements.

Lemma 3.3.6. Let $(A, C)$ be an $O B A$ and $T: A \rightarrow B$ be a Banach algebra homomorphism which satisfies the Riesz property. If $a \in A^{-1} \cap \operatorname{span} C$ and $b \in \mathcal{W}_{T}^{+}$, then $a b, b a \in \mathcal{W}_{T}^{+}$. 
Proof. Since $a \in \operatorname{span} C$, there exist $\lambda_{1}, \lambda_{2}, \ldots, \lambda_{n} \in \mathbb{C}$ and $a_{1}, a_{2}, \ldots, a_{n} \in C$ such that $a=\lambda_{1} a_{1}+\cdots+\lambda_{n} a_{n}$. Now

$$
b a=\left(b_{1}+b_{2}\right) a=b_{1} a+b_{2}\left(\lambda_{1} a_{1}+\cdots+\lambda_{n} a_{n}\right)
$$

for some $b_{1} \in A^{-1}$ and $b_{2} \in C \cap \mathrm{N}(T)$. Using Corollary 3.2.3 we can find $\left(b_{1} a\right)_{n} \in A^{-1}$ and $\mu_{1}, \mu_{2}, \ldots, \mu_{n} \geq 0$ such that

$$
b a=\left(b_{1} a\right)_{n}+\mu_{1} b_{2} a_{1}+\cdots+\mu_{n} b_{2} a_{n} \in A^{-1}+(C \cap \mathrm{N}(T))=\mathcal{W}_{T}^{+} .
$$

In a similar way it can be shown that $a b \in \mathcal{W}_{T}^{+}$.

We remark that, if $a \in A^{-1} \cap C$ in Lemma 3.3.6, then $a b, b a \in \mathcal{W}_{T}^{+}$for an arbitrary $T$.

Unlike the set of Weyl elements relative to an arbitrary Banach algebra homomorphism $T, \mathcal{W}_{T}^{+}$is not in general closed under multiplication. We verify this in the next example.

Example 3.3.7. Consider the $O B A(C(K), C)$, where $K=[0,1]$ and $C:=\{f \in$ $C(K): f(x) \in \mathbb{R}^{+}$for all $\left.x \in K\right\}$, and let $T: C(K) \rightarrow C(K)$ be the homomorphism induced by composition with the unit function 1 on $K$. If $h \in C(K)$ is defined by $h(z)=-z$ for all $z \in K$, then $h \in \mathcal{W}_{T}^{+}$, but $h^{2} \notin \mathcal{W}_{T}^{+}$.

From Example 3.3.5 we have that $h \in \mathcal{W}_{T}^{+}$. A similar approach to that in Example 3.1.8 shows that $h_{\mid \mathbf{1}(K)}^{2}$ has no invertible extension, say, $g$ to $K$ satisfying $z^{2}=h^{2}(z) \geq g(z)$ for all $z \in K$. Hence $h^{2} \notin \mathcal{W}_{T}^{+}$.

Theorem 3.3.8 describes conditions under which the product of upper Weyl elements is again upper Weyl. In particular, from (c) we have that the product of positive upper Weyl elements are again upper Weyl whenever the Banach algebra homomorphism satisfies the Riesz property.

Theorem 3.3.8. Let $(A, C)$ be an $O B A$ and $T: A \rightarrow B$ be a Banach algebra homomorphism. If $a, b \in \mathcal{W}_{T}^{+}$, then, under each of the following assumptions, $a b \in \mathcal{W}_{T}^{+}:$

(a) $T$ is spectral radius preserving,

(b) T satisfies the Riesz property and $\mathrm{N}(T)=\operatorname{span}(C \cap \mathrm{N}(T))$,

(c) $a, b \in C$ and $T$ satisfies the Riesz property.

Proof. The proofs of (a) and (b) are clear from Theorem 3.2.6, which gives $\mathcal{W}_{T}=\mathcal{W}_{T}^{+}$, and the fact that $\mathcal{W}_{T}$ is closed under multiplication. 
(c) By assumption, let $a=a_{1}+a_{2}$ and $b=b_{1}+b_{2}$ for some $a_{1}, b_{1} \in A^{-1}$ and $a_{2}, b_{2} \in C \cap \mathrm{N}(T)$. Hence $a_{1}, b_{1} \in A^{-1} \cap$ span $C$, so that

$$
a b=\left(a_{1}+a_{2}\right) b=a_{1} b+a_{2} b \in \mathcal{W}_{T}^{+}+(C \cap \mathrm{N}(T))=\mathcal{W}_{T}^{+}
$$

by Lemma 3.3.6.

We remark (referring to (a) and (b)) that neither of the assumptions " $T$ is spectral radius preserving" and " $T$ has the Riesz property" can in general be dropped. This can be seen from Example 3.3.7, where the given homomorphism is neither spectral radius preserving (Example 3.1.8 and Theorem 3.2.6 (b)), nor satisfies the Riesz property (Example 3.3.5).

It is known that every almost invertible Fredholm element is Browder (see Proposition 1.5.4). In the last part of this section we investigate the relationship between the subsets $A^{D} \cap \mathcal{F}_{T}$ and $\mathcal{B}_{T}^{+}$of $\mathcal{B}_{T}$, where $T: A \rightarrow B$ denotes a Banach algebra homomorphism. From Propositions 1.5.4 and 3.1.5 it is clear that $\mathcal{B}_{T}^{+} \subseteq A^{D} \cap \mathcal{F}_{T}$ whenever $T$ satisfies the Riesz property. (By Example 3.2.12 this inclusion is strict in general.) However, Example 3.3.5 ( $h$ is upper Browder but not almost invertible Fredholm) indicates that the inclusion is no longer valid if the relevant Banach algebra homomorphism does not satisfy the Riesz property. A natural question would be when an almost invertible Fredholm element is upper Browder. We provide an answer to this question in the following theorem.

Theorem 3.3.9. Let $(A, C)$ be an $O B A, T: A \rightarrow B$ be a Banach algebra homomorphism and $a \in A^{D} \cap \mathcal{F}_{T}$. If $p(a, 0) \in C$, then $a$ is upper Browder.

Proof. Suppose that $a \in A^{D} \cap \mathcal{F}_{T}$. If $0 \notin \sigma(a)$, then the result obviously holds since $a \in A^{-1} \subseteq \mathcal{B}_{T}^{+}$. Therefore, suppose that $0 \in$ iso $\sigma(a)$ and consider the representation $a=a-p+p$, where $p:=p(a, 0)$. By Corollary 2.2.6 we have that $a-p \in A^{-1}$, and from Proposition 1.5.5 and assumption it follows that $p \in C \cap \mathrm{N}(T)$. Hence $a \in \mathcal{B}_{T}^{+}$as $a-p$ and $p$ commute.

Our next example shows that, if the spectral idempotent of an almost invertible Fredholm element $a$ corresponding to 0 is not a positive element, then $a$ may not be upper Browder. 
Example 3.3.10. Consider the homomorphism $T: M_{3}^{u}(\mathbb{C}) \rightarrow \mathbb{C}$ defined by

$$
T\left[\begin{array}{ccc}
x_{11} & x_{12} & x_{13} \\
0 & x_{22} & x_{23} \\
0 & 0 & x_{33}
\end{array}\right]=x_{11}
$$

and $M:=\left(\begin{array}{lll}1 & 1 & 0 \\ 0 & 0 & 1 \\ 0 & 0 & 0\end{array}\right) \in M_{3}^{u}(\mathbb{C})=A$. Then $M \in A^{D} \cap \mathcal{F}_{T}, P(M, 0) \notin M_{3}^{u}\left(\mathbb{R}^{+}\right)$ and $M \notin \mathcal{B}_{T}^{+}$.

Since $0 \notin\{1\}=\sigma(T M)$, we have that $M \in \mathcal{F}_{T}$, and hence $M \in A^{D} \cap \mathcal{F}_{T}$. One can easily verify that $M^{D}=\left(\begin{array}{lll}1 & 1 & 1 \\ 0 & 0 & 0 \\ 0 & 0 & 0\end{array}\right)$, and hence

$$
P(M, 0)=I-M^{D} M=\left(\begin{array}{rrr}
0 & -1 & -1 \\
0 & 1 & 0 \\
0 & 0 & 1
\end{array}\right) \notin M_{3}^{u}\left(\mathbb{R}^{+}\right) .
$$

Suppose that $\left(\begin{array}{lll}1 & 1 & 0 \\ 0 & 0 & 1 \\ 0 & 0 & 0\end{array}\right)=\left(\begin{array}{ccc}1 & 1-x_{12} & -x_{13} \\ 0 & -x_{22} & 1-x_{23} \\ 0 & 0 & -x_{33}\end{array}\right)+\left(\begin{array}{ccc}0 & x_{12} & x_{13} \\ 0 & x_{22} & x_{23} \\ 0 & 0 & x_{33}\end{array}\right) \in A^{-1}+\mathrm{N}(T)$, where $x_{12}, x_{13}, x_{23} \geq 0, x_{22}, x_{33}>0$ and the matrices on the right hand side of the identity commute. As in Example 3.2.12, the commutativity of the two matrices forces the equality $x_{12}+x_{22}=0$. But $x_{12} \geq 0$ and $x_{22}>0$, and therefore $M$ cannot be decomposed as a sum of an invertible element and a positive element in $\mathrm{N}(T)$ which commute. Consequently, $M$ is not upper Browder.

Our next result, which is an immediate consequence of Theorem 3.3.9 and which does not require the domain of the Banach algebra homomorphism to be commutative (as in Corollary 3.2.9(a)), gives conditions under which a Browder element is upper Browder.

Corollary 3.3.11. Let $(A, C)$ be an $O B A$ and $T: A \rightarrow B$ be a Banach algebra homomorphism which satisfies the Riesz property. Then every Browder element a with the property that $p(a, 0) \in C$ is upper Browder.

Proof. The result is clear from Proposition 1.5.4 and Theorem 3.3.9.

The following result shows that the sets of upper Weyl and upper Browder elements can also be used to characterize the radical of a Banach algebra. Since the proof of ([26], Proposition 4.1) can be adapted to prove Proposition 3.3.12, we shall omit its proof.

Proposition 3.3.12. Let $(A, C)$ be an $O B A$ and $T: A \rightarrow B$ be a Banach algebra homomorphism. Then

$$
\operatorname{Rad}(A)=\left\{a \in A: \mathcal{B}_{T}^{+} a \subseteq \mathrm{QN}(A)\right\}=\left\{a \in A: \mathcal{W}_{T}^{+} a \subseteq \mathrm{QN}(A)\right\} .
$$




\subsection{Perturbation results}

In this section we study the behaviour of elements belonging to $\mathcal{W}_{T}^{+}$and $\mathcal{B}_{T}^{+}$under perturbation by elements from a number of sets. It is known that the set of Weyl elements (relative to an arbitrary Banach algebra homomorphism) remains stable under perturbation by null space elements. With Corollary 3.2.3 in mind, we can establish the following perturbation result for the upper Weyl elements.

Proposition 3.4.1. Let $(A, C)$ be an $O B A$ and $T: A \rightarrow B$ be a Banach algebra homomorphism satisfying the Riesz property. Then $\mathcal{W}_{T}^{+}=\mathcal{W}_{T}^{+}+\operatorname{span}(C \cap \mathrm{N}(T))$.

Proof. For the non-trivial inclusion, suppose that $a \in \mathcal{W}_{T}^{+}+\operatorname{span}(C \cap \mathrm{N}(T))$. Then there exist $b \in \mathcal{W}_{T}^{+}$and $c \in \operatorname{span}(C \cap \mathrm{N}(T))$ such that

$$
a=b+c=b_{1}+b_{2}+\lambda_{1} c_{1}+\lambda_{2} c_{2}+\cdots+\lambda_{n} c_{n}
$$

where $b_{1} \in A^{-1}, b_{2} \in C \cap \mathrm{N}(T), \lambda_{1}, \ldots, \lambda_{n} \in \mathbb{C}$ and $c_{1}, \ldots, c_{n} \in C \cap \mathrm{N}(T)$. Utilizing Corollary 3.2.3, we have that $a=b_{n}+b_{2}+\mu_{1} c_{1}+\mu_{2} c_{2}+\cdots+\mu_{n} c_{n}$ for some $b_{n} \in A^{-1}$ and $\mu_{1}, \ldots, \mu_{n} \geq 0$, and hence $a \in \mathcal{W}_{T}^{+}$.

Next, we present an analogue of a result by Mouton and Raubenheimer [see [29], Theorem 2.2 (2 and 3)] for upper Browder and upper Weyl elements. We point out that a key role is played by Theorem 1.1.3.

Proposition 3.4.2. Let $(A, C)$ be an OBA, $T: A \rightarrow B$ be a Banach algebra homomorphism and $a \in A$.

(a) If $x=b+c$, where $b \in \operatorname{Rad}(A)$ and $c \in C \cap \mathrm{N}(T)$, then $a \in \mathcal{W}_{T}^{+} \Rightarrow a+x \in$ $\mathcal{W}_{T}^{+}$. In particular, if $x \in \operatorname{Rad}(A)$, then $a \in \mathcal{W}_{T}^{+}$if and only if $a+x \in \mathcal{W}_{T}^{+}$.

(b) If $x=b+c$, where $b, c \in \operatorname{Comm}^{2}(a), b \in \operatorname{Rad}(A)$ and $c \in C \cap \mathrm{N}(T)$, then $a \in \mathcal{B}_{T}^{+} \Rightarrow a+x \in \mathcal{B}_{T}^{+}$.

Proof. (a) Suppose that $a \in \mathcal{W}_{T}^{+}$. Then $a=a_{1}+a_{2}$ for some $a_{1} \in A^{-1}$ and $a_{2} \in C \cap \mathrm{N}(T)$. Now $a+x=a_{1}+a_{2}+b+c=\left(a_{1}+b\right)+\left(a_{2}+c\right)$, where $a_{2}+c \in C \cap \mathrm{N}(T)$ and $a_{1}+b \in A^{-1}$ by Theorem 1.1.3. Hence $a+x \in \mathcal{W}_{T}^{+}$.

Assume now that $x \in \operatorname{Rad}(A)$. Obviously we only need to prove the converse implication. Suppose that $a+x \in \mathcal{W}_{T}^{+}$. Then there exist $b_{1} \in A^{-1}$ and $b_{2} \in C \cap \mathrm{N}(T)$ such that $a+x=b_{1}+b_{2}$, and hence $a=-x+b_{1}+b_{2} \in$ $A^{-1}+(C \cap \mathrm{N}(T))=\mathcal{W}_{T}^{+}$, by Theorem 1.1.3.

(b) Suppose that $a \in \mathcal{B}_{T}^{+}$. Then $a=a_{1}+a_{2}$, where $a_{1} \in A^{-1}, a_{2} \in C \cap \mathrm{N}(T)$ 
and $a_{1} a_{2}=a_{2} a_{1}$. Since $a_{1}, a_{2} \in \operatorname{Comm}(a)$, we have that $b$ and $c$ commute with both $a_{1}$ and $a_{2}$. Also, $b c=c b$ as the identity $a b=b a$ holds. Now $a+x=a_{1}+a_{2}+b+c=\left(a_{1}+b\right)+\left(a_{2}+c\right)$, where the elements $a_{2}+$ $c \in C \cap \mathrm{N}(T)$ and $a_{1}+b \in A^{-1}$ (by Theorem 1.1.3) commute. Therefore $a+x \in \mathcal{B}_{T}^{+}$.

Observe from Proposition 3.4.2(a) that $\mathcal{W}_{T}^{+}=\mathcal{W}_{T}^{+}+\operatorname{Rad}(A)$.

Remark 3.4.3. We point out that, by applying Proposition 1.2.9, the set $\operatorname{Rad}(A)$ in Proposition 3.4.2(b) can be replaced by $\mathrm{QN}(A)$.

In ([29], Theorem 2.2 (2)) Mouton and Raubenheimer showed that the converse implication in Proposition 3.4.2(a) holds for Weyl elements. The following example illustrates that this is not the case for upper Weyl elements.

Example 3.4.4. Consider the $O B A(C(K), C)$, where $K:=[0,1]$ and $C=\{h \in$ $C(K): h(x) \in \mathbb{R}^{+}$for all $\left.x \in K\right\}$, and let $T: C(K) \rightarrow C(K)$ be the homomorphism induced by composition with the unit function 1 . If $f, g \in C(K)$ are defined by $f(z)=z$ and $g(z)=1-z$ for all $z \in K$, then $g \in \operatorname{Rad}(C(K))+(C \cap \mathrm{N}(T))$ and $f+g \in \mathcal{W}_{T}^{+}$, but $f \notin \mathcal{W}_{T}^{+}$.

Since $C(X)$ is a semisimple OBA, we have that $\operatorname{Rad}(C(K))=\{0\}$. Using the fact that $\mathrm{N}(T)=\{w \in C(K): w(1)=0\}$, it follows that $g \in$ $\operatorname{Rad}(C(K))+(C \cap \mathrm{N}(T))$. Also, $f+g=\mathbf{1} \in C(K)^{-1} \subseteq \mathcal{W}_{T}^{+}$, but $f \notin \mathcal{W}_{T}^{+}$ by Example 3.1.8.

Next, we show that the converse implication in Proposition 3.4.2(a) holds whenever $T$ has the Riesz property. We point out that the proof of this result relies on Proposition 3.4.1.

Theorem 3.4.5. Let $(A, C)$ be an $O B A, T: A \rightarrow B$ be a Banach algebra homomorphism satisfying the Riesz property and $a \in A$. If $x=b+c$, where $b \in \operatorname{Rad}(A)$ and $c \in \operatorname{span}(C \cap \mathrm{N}(T))$, then $a \in \mathcal{W}_{T}^{+}$if and only if $a+x \in \mathcal{W}_{T}^{+}$.

In particular, if $x \in \operatorname{Rad}(A)+(C \cap \mathrm{N}(T))$, then $a \in \mathcal{W}_{T}^{+}$is equivalent to $a+x \in \mathcal{W}_{T}^{+}$.

Proof. If $a \in \mathcal{W}_{T}^{+}$, then $a+x=a_{1}+a_{2}+b+c$ for some $a_{1} \in A^{-1}$ and $a_{2} \in C \cap \mathrm{N}(T)$. In view of Theorem 1.1.3 we have that $a_{1}+b \in A^{-1}$, and hence $a_{1}+b+a_{2} \in \mathcal{W}_{T}^{+}$. Consequently, by recalling Proposition 3.4.1, it follows that $a+x=\left(a_{1}+b+a_{2}\right)+c \in \mathcal{W}_{T}^{+}$. 
Conversely, suppose that $a \in A$ is such that $a+x \in \mathcal{W}_{T}^{+}$. Then $a=a+x-x=d_{1}+d_{2}-(b+c)=d_{1}+d_{2}-b+\lambda_{1} c_{1}+\lambda_{2} c_{2}+\cdots+\lambda_{n} c_{n}$ for some $d_{1} \in A^{-1}$ and $d_{2} \in C \cap \mathrm{N}(T)$ and where $\lambda_{1}, \ldots, \lambda_{n} \in \mathbb{C}$ and $c_{1}, \ldots, c_{n} \in C \cap \mathrm{N}(T)$. Consequently, by Corollary 3.2.3, we can find $d_{1 n} \in$ $A^{-1}$ and $\mu_{1}, \ldots, \mu_{n} \geq 0$ satisfying

$$
a=\left(d_{1 n}-b\right)+\left(d_{2}+\mu_{1} c_{1}+\mu_{2} c_{2}+\cdots+\mu_{n} c_{n}\right),
$$

so that $a \in A^{-1}+(C \cap \mathrm{N}(T))=\mathcal{W}_{T}^{+}$in view of Theorem 1.1.3.

The second statement follows directly from the first part of the theorem.

\subsection{Regularities}

Among other things, the theory of regularities can be used to establish spectral mapping theorems for several kinds of spectra. Here, we present examples demonstrating that the sets of upper Weyl and upper Browder elements are not regularities in general.

We start this section by recalling the definition of a regularity given by Kordula and Müller in [23].

Definition 3.5.1 (Regularity). ([23], Definition 1.2) A non-empty subset $R$ of a Banach algebra $A$ is called a regularity if the following properties are satisfied:

(a) if $a \in A$ and $n \in \mathbb{N}$, then $a \in R$ if and only if $a^{n} \in R$,

(b) if $a, b, c, d \in A$ are mutually commuting elements such that $a c+b d=\mathbf{1}$, then $a, b \in R$ if and only if $a b \in R$.

Examples of some of the more well-known regularities include the sets of left and right invertible elements and the set of invertible elements of a Banach algebra. It is familiar that the sets of Browder and Weyl elements relative to an arbitrary Banach algebra homomorphism are not regularities in general.

In [33] Müller divided the axioms of the definition of a regularity into two parts. This process gave rise to two new notions: the concepts of lower and upper semi-regularities.

Definition 3.5.2 (Lower semi-regularity). ([33], Definition 1) A non-empty subset $R$ of a Banach algebra $A$ is called a lower semi-regularity if the following 
properties are satisfied:

(a) if $a \in A, n \in \mathbb{N}$ and $a^{n} \in R$ implies $a \in R$,

(b) if $a, b, c, d \in A$ are mutually commuting elements such that $a c+b d=\mathbf{1}$, then $a b \in R$ implies $a, b \in R$.

Definition 3.5.3 (Upper semi-regularity). ([33], Definition 10) A non-empty subset $R$ of a Banach algebra $A$ is called an upper semi-regularity if the following properties are satisfied:

(a) if $a \in A$, then $a \in R$ implies that $a^{n} \in R$ for all $n \in \mathbb{N}$,

(b) if $a, b, c, d \in A$ are mutually commuting elements such that $a c+b d=\mathbf{1}$, then

$a, b \in R$ implies $a b \in R$,

(c) $R$ contains a neighbourhood of the unit $\mathbf{1}$.

Consequently, a subset of a Banach algebra is a regularity if and only if it is both an upper and a lower semi-regularity.

We remark that condition (c) in Definition 3.5.3 is automatically satisfied by lower semi-regularities as a lower semi-regularity always contains the open set of invertible elements ([33], Lemma 2(ii)).

Proposition 3.5.4. Let $T: A \rightarrow B$ be a Banach algebra homomorphism. Then $\mathcal{W}_{T}$ is an upper semi-regularity.

Moreover, if $T$ has the Riesz property, then $\mathcal{B}_{T}$ is an open regularity.

The first statement above is clear from ([28], Corollary 8.2). For $\mathcal{B}_{T}$ the result follows from the fact that $\mathcal{B}_{T}=\mathcal{B}_{\pi}$ ([28], Corollary 5.6), ([26], Theorem 7.5) and the identity $\mathcal{B}_{\pi}=A^{D} \cap \mathcal{F}_{\pi}$, where $\pi: A \rightarrow A / \overline{\mathrm{N}(T)}$ denotes the canonical homomorphism.

In ([28], pp.354-355) H. Mouton, S. Mouton and H. Raubenheimer remarked (referring to ([21], Example 4.4)) that $\mathcal{W}_{T}$ is not a lower semi-regularity in general (even if $T$ has the Riesz property).

In Examples 3.5.5 and 3.5.6 we demonstrate that the sets of upper Browder and upper Weyl elements of a Banach algebra are in general neither lower nor upper semi-regularities.

Example 3.5.5. Consider the $O B A(C(K), C)$, where $K:=[0,1]$ and $C=\{h \in$ $C(K): h(x) \in \mathbb{R}^{+}$for all $\left.x \in K\right\}$, and let $T: C(K) \rightarrow C(K)$ be the homomorphism induced by composition with the unit function 1 . Then $\mathcal{W}_{T}^{+}\left(=\mathcal{B}_{T}^{+}\right)$is not a lower semi-regularity. 
Consider $h \in C(K)$ defined by $h(z)=z$ for all $z \in K$. Then $h,-\mathbf{1}, 0,-\mathbf{1} \in$ $C(K)$ are mutually commuting functions satisfying $h 0+(-\mathbf{1})(-\mathbf{1})=\mathbf{1}$. Using Example 3.3.5 we have that $-h=h(-\mathbf{1}) \in \mathcal{W}_{T}^{+}$, but $h \notin \mathcal{W}_{T}^{+}$by Example 3.1.8.

Example 3.5.6. Consider the $O B A(C(K), C)$, where $K:=[0,1]$ and $C=\{h \in$ $C(K): h(x) \in \mathbb{R}^{+}$for all $\left.x \in K\right\}$, and let $T: C(K) \rightarrow C(K)$ be the homomorphism induced by composition with the unit function 1 . Then $\mathcal{W}_{T}^{+}\left(=\mathcal{B}_{T}^{+}\right)$is not an upper semi-regularity.

Consider $h \in C(K)$ defined by $h(z)=-z$ for all $z \in K$. The result is clear from Example 3.3.7.

In view of ([4], Theorem 3) and the remark in the second paragraph following Proposition 3.5.4 we have (even for a homomorphism $T$ which satisfies the Riesz property) that $\mathcal{W}_{T}^{+}$is not a lower semi-regularity in general.

A consequence of Theorem 3.2.6(a), Corollary 3.2.9(a) and Proposition 3.5.4 is the following.

Corollary 3.5.7. Let $(A, C)$ be an $O B A$ and $T: A \rightarrow B$ be a Banach algebra homomorphism satisfying the Riesz property and $\mathrm{N}(T)=\operatorname{span}(C \cap \mathrm{N}(T))$. Then $\mathcal{W}_{T}^{+}$is an upper semi-regularity.

Furthermore, if $A$ is also commutative, then $\mathcal{W}_{T}^{+}\left(=\mathcal{B}_{T}^{+}\right)$is an open regularity. 


\section{Chapter 4}

\section{The upper Browder and upper Weyl spectra}

Throughout this chapter, let $(A, C)$ denote an arbitrary OBA and $T: A \rightarrow$ $B$ a Banach algebra homomorphism. Just as the sets $\mathcal{W}_{T}$ and $\mathcal{B}_{T}$ define, respectively, the Weyl and Browder spectra w.r.t. $T$, we use here the sets $\mathcal{W}_{T}^{+}$and $\mathcal{B}_{T}^{+}$to define two new spectra: the upper Weyl and upper Browder spectra (relative to $T$ ). The aim of this chapter is to study the basic properties of the upper Weyl and upper Browder spectra of an element in an OBA.

\subsection{Elementary properties and examples}

Definition 4.1.1. Let $(A, C)$ be an $O B A, T: A \rightarrow B$ be a Banach algebra homomorphism and $a \in A$.

- The upper Browder spectrum of a, denoted by $\beta_{T}^{+}(a)$, is given by

$$
\beta_{T}^{+}(a)=\left\{\lambda \in \mathbb{C}: \lambda \mathbf{1}-a \notin \mathcal{B}_{T}^{+}\right\} .
$$

- The upper Weyl spectrum of a, denoted by $\omega_{T}^{+}(a)$, is given by

$$
\omega_{T}^{+}(a)=\left\{\lambda \in \mathbb{C}: \lambda \mathbf{1}-a \notin \mathcal{W}_{T}^{+}\right\} .
$$

Since $\mathcal{W}_{T}\left(\mathcal{B}_{T}\right)$ is closed under non-zero scalar multiplication, it follows that $\lambda \mathbf{1}-a$ is Weyl (Browder) if and only if $a-\lambda \mathbf{1}$ is Weyl (Browder). A consequence of the fact that $\mathcal{W}_{T}^{+}$and $\mathcal{B}_{T}^{+}$are not closed under non-zero scalar multiplication (recall Example 3.3.5) is that the sets

$$
\{\lambda \in \mathbb{C}: \lambda \mathbf{1}-a \notin X\} \text { and }\{\lambda \in \mathbb{C}: a-\lambda \mathbf{1} \notin X\},
$$


where $X \in\left\{\mathcal{B}_{T}^{+}, \mathcal{W}_{T}^{+}\right\}$, do not coincide in general. We verify this in the next example.

Example 4.1.2. Consider the $O B A(C(K), C)$, where $K:=[0,1]$ and $C=\{h \in$ $C(K): h(x) \in \mathbb{R}^{+}$for all $\left.x \in K\right\}$, and let $T: C(K) \rightarrow C(K)$ be the homomorphism induced by composition with the unit function 1. If $f \in C(K)$ is defined by $f(z)=z$ for all $z \in K$, then $\omega_{T}^{+}(f)=\{1\}$ and $\left\{\lambda \in \mathbb{C}: f-\lambda \mathbf{1} \notin \mathcal{W}_{T}^{+}\right\}=$ $[0,1]$. Therefore

$$
\beta_{T}^{+}(f)=\omega_{T}^{+}(f) \neq\left\{\lambda \in \mathbb{C}: f-\lambda \mathbf{1} \notin \mathcal{W}_{T}^{+}\right\}=\left\{\lambda \in \mathbb{C}: f-\lambda \mathbf{1} \notin \mathcal{B}_{T}^{+}\right\} .
$$

Recall from Example 3.1.4 that $\lambda \mathbf{1}-f$ is upper Weyl w.r.t. $T$ if and only if $(\lambda \mathbf{1}-f)_{\mid \mathbf{1}(K)}$ has an invertible extension to $K$, say $g$, satisfying $\lambda \mathbf{1}-f \geq g$.

If $\lambda=1$, then $(\lambda \mathbf{1}-f)_{\mid \mathbf{1}(K)}(1)=0$, so that $(\lambda \mathbf{1}-f)_{\mid \mathbf{1}(K)}$ is not invertible, and hence does not have an invertible extension to $K$. This gives $1 \in \omega_{T}^{+}(f)$.

If $\lambda \neq 1$, then $(\lambda \mathbf{1}-f)_{\mid \mathbf{1}(K)}(1)=\lambda-1$, and hence $0 \notin(\lambda \mathbf{1}-f)_{\mid \mathbf{1}(K)}(\{1\})$, so that $(\lambda \mathbf{1}-f)_{\mid \mathbf{1}(K)}$ is invertible. Consider the constant function $g: K \rightarrow \mathbb{C}$ defined by $g(z)=\lambda-1$ for all $z \in K$. Then $g$ is an invertible extension of $(\lambda \mathbf{1}-f)_{\mid \mathbf{1}(K)}$ to $K$ satisfying $\lambda \mathbf{1}-f \geq g$. Hence $\lambda \notin \omega_{T}^{+}(f)$. This gives $\omega_{T}^{+}(f)=\{1\}$.

Again, $f-\lambda \mathbf{1}$ is upper Weyl w.r.t. $T$ if and only if $(f-\lambda \mathbf{1})_{\mid \mathbf{1}(K)}$ has an invertible extension to $K$, say $g$, satisfying $f-\lambda \mathbf{1} \geq g$.

A similar approach (using the intermediate value theorem) to that in the proof of Example 3.1.8 shows that, for $\lambda \in[0,1]$, the function $(f-\lambda \mathbf{1})_{\mid \mathbf{1}(K)}$ has no invertible extension $g$ to $K$ satisfying $f-\lambda \mathbf{1} \geq g$. Hence $f-\lambda \mathbf{1}$ is not upper Weyl. By also using the fact that $A^{-1} \subseteq \mathcal{W}_{T}^{+}$, it follows that

$$
[0,1] \subseteq\left\{\lambda \in \mathbb{C}: f-\lambda \mathbf{1} \notin \mathcal{W}_{T}^{+}\right\} \subseteq \sigma(f)=[0,1],
$$

and hence $\omega_{T}^{+}(f)=\{1\} \neq[0,1]=\left\{\lambda \in \mathbb{C}: f-\lambda \mathbf{1} \notin \mathcal{W}_{T}^{+}\right\}$. The result follows from the fact that $C(K)$ is commutative.

In view of Lemma 3.3.4 we have the following:

Proposition 4.1.3. Let $(A, C)$ be an $O B A$ and $T: A \rightarrow B$ be a Banach algebra homomorphism which satisfies the Riesz property. If $a \in A$, then

$$
\omega_{T}^{+}(a)=\left\{\lambda \in \mathbb{C}: \lambda \mathbf{1}-a \notin \mathcal{W}_{T}^{+}\right\}=\left\{\lambda \in \mathbb{C}: a-\lambda \mathbf{1} \notin \mathcal{W}_{T}^{+}\right\}=-\omega_{T}^{+}(-a)
$$

and

$$
\beta_{T}^{+}(a)=\left\{\lambda \in \mathbb{C}: \lambda \mathbf{1}-a \notin \mathcal{B}_{T}^{+}\right\}=\left\{\lambda \in \mathbb{C}: a-\lambda \mathbf{1} \notin \mathcal{B}_{T}^{+}\right\}=-\beta_{T}^{+}(-a) .
$$


The following result shows that the upper Weyl and upper Browder spectra give rise to "essential spectra". The proof is fairly straightforward, but we include it for the sake of completeness.

Proposition 4.1.4. Let $(A, C)$ be an $O B A$ and $T: A \rightarrow B$ be a Banach algebra homomorphism. For $a \in A$, we have the following equalities:

$$
\begin{aligned}
& \beta_{T}^{+}(a)=\bigcap_{\substack{c \in C \cap N(T) \\
a c=c a}} \sigma(a+c) \\
& \omega_{T}^{+}(a)=\bigcap_{c \in C \cap \mathrm{N}(T)} \sigma(a+c) \\
& \left\{\lambda \in \mathbb{C}: a-\lambda \mathbf{1} \notin \mathcal{B}_{T}^{+}\right\}=\bigcap_{\substack{c \in C \cap N(T) \\
a c=c a}} \sigma(a-c)=\bigcap_{\substack{c \in-C \cap N(T) \\
a c=c a}} \sigma(a+c) \\
& \left\{\lambda \in \mathbb{C}: a-\lambda \mathbf{1} \notin \mathcal{W}_{T}^{+}\right\}=\bigcap_{c \in C \cap N(T)} \sigma(a-c)=\bigcap_{c \in-C \cap N(T)} \sigma(a+c)
\end{aligned}
$$

Proof. We first prove (4.1.5). Suppose that $\lambda \notin \bigcap_{c \in C \cap N(T)} \sigma(a+c)$. Then there exists $d \in C \cap \mathrm{N}(T)$ commuting with $a$ such that $\lambda \notin \sigma(a+d)$. Let $b:=\lambda \mathbf{1}-(a+d)$. Then $\lambda \mathbf{1}-a=b+d$, where $b \in A^{-1}$ and $d \in C \cap \mathrm{N}(T)$ satisfy $b d=d b$. Hence $\lambda \mathbf{1}-a \in \mathcal{B}_{T}^{+}$, so that $\lambda \notin \beta_{T}^{+}(a)$. Consequently,

$$
\beta_{T}^{+}(a) \subseteq \bigcap_{\substack{c \in C \cap \mathrm{N}(T) \\ a c=c a}} \sigma(a+c) .
$$

Conversely, suppose that $\lambda \notin \beta_{T}^{+}(a)$. Then $\lambda \mathbf{1}-a \in \mathcal{B}_{T}^{+}$, and hence there exist commuting elements $b \in A^{-1}$ and $d \in C \cap \mathrm{N}(T)$ such that $\lambda \mathbf{1}-a=$ $b+d$. Note that $d$ also commutes with $a$ and that $\lambda \mathbf{1}-(a+d)=b \in A^{-1}$. Hence $\lambda \notin \sigma(a+d)$, so that $\lambda \notin \bigcap_{c \in C \cap N(T)} \sigma(a+c)$. Therefore

$$
\beta_{T}^{+}(a) \supseteq \bigcap_{\substack{c \in C \cap N(T) \\ a c=c a}} \sigma(a+c)
$$

and the equality is proved.

By following a similar argument as in the proof of (4.1.5), the equality in (4.1.6) can be obtained.

Also, a similar approach as in the proof of (4.1.5) can be used to prove (4.1.7) and (4.1.8). 
We observe that the difference between the upper Browder and upper Weyl spectra and the sets in (4.1.7) and (4.1.8), respectively, is that the set $C \cap \mathrm{N}(T)$ in (4.1.5) and (4.1.6) is replaced by the set $-C \cap \mathrm{N}(T)$.

Remark 4.1.9. If $E$ denotes a Banach lattice, then the upper Weyl spectrum of $T \in \mathcal{L}(E)$ (relative to $\pi: \mathcal{L}(E) \rightarrow \mathcal{L}(E) / \mathcal{K}(E)$ ) was introduced by Alekhno in [3], as in (4.1.6), without utilizing the concept of an upper Weyl element.

Corollary 4.1.10. Let $(A, C)$ be an $O B A$ and $T: A \rightarrow B$ be a Banach algebra homomorphism which satisfies the Riesz property. Then

$$
\beta_{T}^{+}(a)=\bigcap_{\substack{c \in C \cap N(T) \\ a c=c a}} \sigma(a-c)
$$

and

$$
\bigcap_{c \in \mathrm{C} \cap \mathrm{N}(T)} \sigma(a-c)=\omega_{T}^{+}(a)=\bigcap_{c \in \operatorname{span}(\mathrm{C \cap N}(T))} \sigma(a+c)
$$

for all $a \in A$.

Proof. The identity for the upper Browder spectrum and the first identity for the upper Weyl spectrum follow from Proposition 4.1.3 and equations (4.1.7) and (4.1.8). To establish the second identity for the upper Weyl spectrum, we need only show that $\omega_{T}^{+}(a) \subseteq \bigcap_{c \in \operatorname{span}(C \cap N(T))} \sigma(a+c)$. Hence suppose that $\lambda \notin \bigcap_{c \in \operatorname{span}(C \cap \mathrm{N}(T))} \sigma(a+c)$. Then there exists $d \in \operatorname{span}(C \cap \mathrm{N}(T))$ such that $\lambda \notin \sigma(a+d)$; that is, $\lambda \mathbf{1}-a-d \in A^{-1} \subseteq \mathcal{W}_{T}^{+}$in view of Proposition 3.1.5, so that $\lambda \mathbf{1}-a \in \mathcal{W}_{T}^{+}$by Proposition 3.4.1. Thus, $\lambda \notin \omega_{T}^{+}(a)$.

Proposition 4.1.11. Let $(A, C)$ be an $O B A$ and $T: A \rightarrow B$ be a Banach algebra homomorphism. If $a \in A$, then

$$
\begin{aligned}
& \omega_{T}^{+}(a)
\end{aligned}
$$

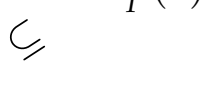

$$
\begin{aligned}
& \sigma(T a) \subseteq \omega_{T}(a) \\
& \leqslant \\
& \beta_{T}(a)
\end{aligned}
$$

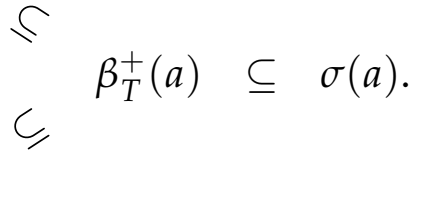

Proof. This follows directly from Proposition 3.1.5.

Corollary 4.1.12. Let $(A, C)$ be an $O B A$ and $T: A \rightarrow B$ be a Banach algebra homomorphism. If $a \in A$, then the sets $\beta_{T}^{+}(a)$ and $\omega_{T}^{+}(a)$ are non-empty and compact. 
4.1. Elementary properties and examples

Proof. The non-empty property follows from the fact that $\sigma(\mathrm{Ta})$ is non-empty and Proposition 4.1.11, while the compact property follows from the fact that the spectrum is a non-empty compact (Theorem 1.2.8 (ii)) subset of a Hausdorff space, and Proposition 4.1.4.

Examples 3.1.6 to 3.1.8 indicate that the inclusions in Proposition 4.1.11 are strict in general. The examples presented next illustrate this fact again, but here, for each of our examples, we describe what each set in Proposition 4.1.11 looks like. This information will be useful in the sequel.

Example 4.1.13. Consider the $O B A(C(K), C)$, where $K:=[0,1]$ and $C=\{h \in$ $C(K): h(x) \in \mathbb{R}^{+}$for all $\left.x \in K\right\}$, and let $T: C(K) \rightarrow C(K)$ be the homomorphism induced by composition with the unit function 1 . If $f \in C(K)$ is defined by $f(z)=z$ for all $z \in K$, then

$$
\begin{aligned}
& \omega_{T}^{+}(-f) \\
& \sigma(T(-f))=\omega_{T}(-f)
\end{aligned}
$$

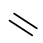

$$
\begin{aligned}
& \beta_{T}^{+}(-f)=\sigma(-f), \\
& \beta_{T}(-f)
\end{aligned}
$$

where $\beta_{T}(-f)=\{-1\}$ and $\sigma(-f)=[-1,0]$.

Directly from Example 3.1.8 we have that $0 \in \omega_{T}^{+}(-f) \backslash \omega_{T}(-f)$. Moreover, a similar argument as in Example 4.1.2 yields $\omega_{T}(-f)=\{-1\}$, and hence

$$
\{-1\}=\omega_{T}(-f) \subseteq \omega_{T}^{+}(-f) \subseteq \sigma(-f)=[-1,0]
$$

in view of Proposition 4.1.11. It remains to use a similar argument as in Example 3.1.8 to obtain $\omega_{T}^{+}(-f)=[-1,0]$. The result then follows from the fact that $C(K)$ is commutative and the fact that $\sigma(T(-f)) \neq \varnothing$.

Our next example illustrates that both the Browder and upper Weyl spectra are in general strictly contained in the upper Browder spectrum.

Example 4.1.14. Consider the homomorphism $T: M_{3}^{u}(\mathbb{C}) \rightarrow \mathbb{C}$ defined by

$$
T\left[\begin{array}{ccc}
x_{11} & x_{12} & x_{13} \\
0 & x_{22} & x_{23} \\
0 & 0 & x_{33}
\end{array}\right]=x_{11}
$$

and $M:=\left(\begin{array}{lll}1 & 1 & 0 \\ 0 & 0 & 1 \\ 0 & 0 & 0\end{array}\right) \in M_{3}^{u}(\mathbb{C})=A$. Then 


$$
\begin{aligned}
& \omega_{T}^{+}(M) \\
& \sigma(T M)=\omega_{T}(M) \\
& \S \beta_{T}^{+}(M)=\sigma(M), \\
& \beta_{T}(M)
\end{aligned}
$$

where $\beta_{T}(M)=\{1\}$ and $\sigma(M)=\{0,1\}$.

We have that $\lambda \notin \omega_{T}^{+}(M)$ if and only if

$$
\left(\begin{array}{ccc}
\lambda-1 & -1 & 0 \\
0 & \lambda & -1 \\
0 & 0 & \lambda
\end{array}\right)=\lambda\left(\begin{array}{lll}
1 & 0 & 0 \\
0 & 1 & 0 \\
0 & 0 & 1
\end{array}\right)-\left(\begin{array}{lll}
1 & 1 & 0 \\
0 & 0 & 1 \\
0 & 0 & 0
\end{array}\right) \in \mathcal{W}_{T}^{+} ;
$$

which happens if and only if $\lambda \neq 1$ (as established in Example 3.2.12). Hence

$$
\{1\}=\omega_{T}^{+}(M) \subseteq \beta_{T}^{+}(M) \subseteq \sigma(M)=\{0,1\}
$$

in view of Proposition 4.1.11. Since $M \notin \mathcal{B}_{T}^{+}$by Example 3.3.10, we have from Proposition 4.1.3 and (4.1.15) that $\beta_{T}^{+}(M)=\{0,1\}$. The result then follows from Example 3.2.12 and equation (4.1.15).

We point out here that another example illustrating that the upper Weyl spectrum of an OBA element is strictly contained in the upper Browder spectrum of that element is provided by Example 3.1.7.

Example 4.1.16. Consider the homomorphism $T: \mathbb{C}^{2} \rightarrow \mathbb{C}$ defined by $T(z, w)=$ $z$ and $a:=(1,0) \in \mathbb{C}^{2}$. Then

$$
\begin{aligned}
& \omega_{T}^{+}(a) \\
& \sigma(T a)=\omega_{T}(a) \\
& \beta_{T}^{+}(a) \subsetneq \sigma(a), \\
& \beta_{T}(a)
\end{aligned}
$$

where $\beta_{T}^{+}(a)=\{1\}$ and $\sigma(a)=\{0,1\}$.

From Example 3.2.10 we have that $\lambda(1,1)-(1,0)=(\lambda-1, \lambda) \in \mathcal{B}_{T}^{+}(=$ $\left.\mathcal{W}_{T}^{+}\right)$if and only if $\lambda \neq 1$, and hence $\beta_{T}^{+}(a)=\{1\} \subsetneq\{0,1\}=\sigma(a)$. The result follows from the fact that all the other spectra in the diagram are nonempty.

We conclude this section by presenting a few perturbation results for the upper Weyl spectrum. These corollaries are immediate consequences of results established in Section 3.4. 
Corollary 4.1.17. Let $(A, C)$ be an $O B A$ and $T: A \rightarrow B$ be a Banach algebra homomorphism. If $a \in A$, then $\omega_{T}^{+}(a)=\omega_{T}^{+}(a+b)$ for all $b \in \operatorname{Rad}(A)$.

Proof. Let $a \in A$ and $b \in \operatorname{Rad}(A)$. Then $\lambda \notin \omega_{T}^{+}(a)$ if and only if $\lambda \mathbf{1}-a \in$ $\mathcal{W}_{T}^{+}$, which is equivalent to $\lambda \mathbf{1}-a+(-b) \in \mathcal{W}_{T}^{+}$by Proposition 3.4.2(a); that is $\lambda \notin \omega_{T}^{+}(a+b)$. The proof is complete.

It is interesting to note that another proof of the above fact can be obtained using ([10], Theorem 5.3.1).

The following example demonstrates that Corollary 4.1.17 is not true for the upper Browder spectrum.

Example 4.1.18. Let $A:=M_{2}^{u}(\mathbb{C})$ and consider the homomorphism $T: A \rightarrow \mathbb{C}$ defined by $T\left[\begin{array}{cc}x_{11} & x_{12} \\ 0 & x_{22}\end{array}\right]=x_{11}$. If $a:=\left(\begin{array}{ll}1 & 1 \\ 0 & 0\end{array}\right) \in A$, then $\beta_{T}^{+}(a) \neq \beta_{T}^{+}(a+b)$ for $b=\left(\begin{array}{cc}0 & -2 \\ 0 & 0\end{array}\right) \in \operatorname{Rad}(A)$.

It is clear that $\sigma(T a)=\{1\}$ and $\sigma(a)=\{0,1\}$. We show that $a \notin \mathcal{B}_{T}^{+}$. Suppose that this is not the case. Then there exist $a_{1}:=\left(\begin{array}{cc}1 & 1-x_{12} \\ 0 & -x_{22}\end{array}\right) \in A^{-1}$, (that is, $\left.x_{22} \neq 0\right)$ and $a_{2}:=\left(\begin{array}{ll}0 & x_{12} \\ 0 & x_{22}\end{array}\right) \in M_{2}^{u}\left(\mathbb{R}^{+}\right) \cap \mathrm{N}(T)$ such that $a_{1} a_{2}=a_{2} a_{1}$. The commutativity property, however, implies that $x_{12}+x_{22}=0$, which is impossible as $x_{22}>0$. Therefore $a \notin \mathcal{B}_{T}^{+}$, and hence $0 \in \beta_{T}^{+}(a)$ by Proposition 4.1.3. Consequently, $\beta_{T}^{+}(a)=\{0,1\}$ using Proposition 4.1.11.

Now, $a+b:=\left(\begin{array}{cc}1 & -1 \\ 0 & 0\end{array}\right)$, and hence

$$
\{1\}=\sigma(T(a+b)) \subseteq \beta_{T}^{+}(a+b) \subseteq \sigma(a+b)=\{0,1\}
$$

by using Proposition 4.1.11 again. Since

$$
\left(\begin{array}{cc}
1 & -1 \\
0 & 0
\end{array}\right)=\left(\begin{array}{ll}
1 & -2 \\
0 & -1
\end{array}\right)+\left(\begin{array}{ll}
0 & 1 \\
0 & 1
\end{array}\right) \in A^{-1}+\left(M_{2}^{u}\left(\mathbb{R}^{+}\right) \cap \mathrm{N}(T)\right),
$$

where the two matrices on the right hand side commute, we have that $a+b \in \mathcal{B}_{T}^{+}$. Hence $0 \notin \beta_{T}^{+}(a+b)$ by Proposition 4.1 .3 , so that $\beta_{T}^{+}(a+b)=$ $\{1\} \neq\{0,1\}=\beta_{T}^{+}(a)$.

The following result indicates that the upper Weyl spectrum (relative to Banach algebra homomorphisms satisfying the Riesz property) is invariant under perturbation of positive and negative null space elements.

Corollary 4.1.19. Let $(A, C)$ be an $O B A$ and $T: A \rightarrow B$ be a Banach algebra homomorphism which satisfies the Riesz property. If $a \in A$, then $\omega_{T}^{+}(a)=$ $\omega_{T}^{+}(a+b)$ for all $b \in \operatorname{span}(C \cap \mathrm{N}(T))$. 
Proof. Let $a \in A$ and $b \in \operatorname{span}(C \cap \mathrm{N}(T))$. In view of the representation of the upper Weyl spectrum according to Corollary 4.1.10, it follows that

$\omega_{T}^{+}(a+b)=\bigcap_{c \in \operatorname{span}(C \cap \mathrm{N}(T))} \sigma(a+b+c)=\bigcap_{d \in \operatorname{span}(\mathrm{C} \cap \mathrm{N}(T))} \sigma(a+d)=\omega_{T}^{+}(a)$, which concludes the proof.

\subsection{Spectral mapping theorems}

In this section we investigate whether the spectra introduced in Definition 4.1.1 obey any spectral mapping theorems. We recall the following spectral inclusion theorem by Harte for the Weyl and Browder spectra.

Theorem 4.2.1. ([20], Theorem 2) Let $T: A \rightarrow B$ be a Banach algebra homomorphism, $a \in A$ and $f: U \rightarrow \mathbb{C}$ be a holomorphic function on $U$, an open set containing $\sigma(a)$. If $f$ is non-constant on every component of $U$, then

$$
\omega_{T}(f(a)) \subseteq f\left(\omega_{T}(a)\right)
$$

and

$$
\beta_{T}(f(a)) \subseteq f\left(\beta_{T}(a)\right) .
$$

Moreover, if T satisfies the Riesz property, then $\beta_{T}(f(a))=f\left(\beta_{T}(a)\right)$.

It is worth noting that, since $\mathcal{B}_{T}$ is a regularity whenever $T$ has the Riesz property and $\mathcal{W}_{T}$ is an upper semiregularity (recall Proposition 3.5.4), the above theorem was recently proved in a more general context (see [[33], Theorem 20] and [[23], Theorem 1.4]).

We begin by observing that, under the assumptions of Theorem 4.2.1, the upper Weyl and upper Browder spectra do not satisfy the given oneway spectral inclusions.

Example 4.2.2. Consider the $O B A(C(K), C)$, where $K=[0,1]$ and $C:=\{h \in$ $C(K): h(x) \in \mathbb{R}^{+}$for all $\left.x \in K\right\}$, and let $T: C(K) \rightarrow C(K)$ be the homomorphism induced by composition with the unit function 1 on K. Consider the element $f \in C(K)$ defined by $f(z)=z$ for all $z \in K$ and let $U$ be an open set containing $\sigma(f):=[0,1]$. Then

$$
\beta_{T}^{+}(g(f))=\omega_{T}^{+}(g(f))=[-1,0] \nsubseteq\{-1\}=g\left(\omega_{T}^{+}(f)\right)=g\left(\beta_{T}^{+}(f)\right),
$$

where $g: U \rightarrow \mathbb{C}$ defined by $g(\lambda)=-\lambda$ is holomorphic on $U$ and non-constant on every component of $U$. 
From Example 4.1.13 we have that $\omega_{T}^{+}(g(f))=\omega_{T}^{+}(-f)=[-1,0]$, while Example 4.1.2 gives $g\left(\omega_{T}^{+}(f)\right)=g(\{1\})=\{-1\}$. The result follows from the fact that $C(K)$ is commutative.

Using Theorem 3.2.6(a), Corollary 3.2.9(a) and Theorem 4.2.1, we have the following spectral inclusion results.

Proposition 4.2.3. Let $(A, C)$ be an $O B A$ and $T: A \rightarrow B$ be a Banach algebra homomorphism with the Riesz property satisfying $\mathrm{N}(T)=\operatorname{span}(C \cap \mathrm{N}(T))$. If $a \in A$ and $U$ is an open set containing $\sigma(a)$, then

$$
\omega_{T}^{+}(f(a)) \subseteq f\left(\omega_{T}^{+}(a)\right)
$$

for every function $f: U \rightarrow \mathbb{C}$ holomorphic on $U$ which is non-constant on each component of $U$.

Moreover, if $A$ is also commutative, then

$$
\beta_{T}^{+}(f(a))=f\left(\beta_{T}^{+}(a)\right) .
$$

In establishing Theorem 4.2.1 an important role was played by the fact that $\mathcal{W}_{T}$ is closed under multiplication. As this is not the case for $\mathcal{W}_{T}^{+}$in general (recall Example 3.3.7), it is not easy to set up a one-way spectral inclusion result (designed for the situation $\mathcal{W}_{T}^{+} \subsetneq \mathcal{W}_{T}$ ) for the upper Weyl spectrum. In particular, we emphasize that the approach (relying on ([16], Theorem 3.7 and Corollary 3.9, pp.78-79)) followed by Harte to proof Theorem 4.2.1 is of no use here.

Unfortunately, at this stage, we have not yet managed (for cases where $\mathcal{W}_{T}^{+} \subsetneq \mathcal{W}_{T}$ and $\mathcal{B}_{T}^{+} \subsetneq \mathcal{B}_{T}$ ) to come up with spectral inclusion results for the upper Weyl and upper Browder spectra dealing with more general holomorphic functions and arbitrary (positive) elements. However, we provide an answer (see Proposition 4.2.5) in the case where the function $f(\lambda)=\lambda^{-1}$ is considered.

It is known that $\left(\omega_{T}(a)\right)^{-1}=\omega_{T}\left(a^{-1}\right)$ and $\left(\beta_{T}(a)\right)^{-1}=\beta_{T}\left(a^{-1}\right)$ for all $a \in A^{-1}$. Our next example illustrates that even these identities do not in general hold for the upper Weyl and upper Browder spectra.

Example 4.2.4. Consider the $O B A(C(K), C)$, where $K:=\left[\frac{1}{2}, 1\right]$ and $C=\{h \in$ $C(K): h(x) \in \mathbb{R}^{+}$for all $\left.x \in K\right\}$, and let $T: C(K) \rightarrow C(K)$ be the homomorphism induced by composition with the unit function 1 . Consider the invertible element $f \in C(K)$ defined by $f(z)=z$ for all $z \in K$. Then

$$
\left(\beta_{T}^{+}(f)\right)^{-1}=\left(\omega_{T}^{+}(f)\right)^{-1}=\{1\} \neq[1,2]=\omega_{T}^{+}\left(f^{-1}\right)=\beta_{T}^{+}\left(f^{-1}\right) .
$$


As in the proof of Example 4.1.2 we get that $\omega_{T}^{+}(f)=\{1\}$, and hence $\left(\omega_{T}^{+}(f)\right)^{-1}=\{1\}$. Recalling Proposition 4.1.11 it follows that $\omega_{T}\left(f^{-1}\right)=$ $\left(\omega_{T}(f)\right)^{-1}=\{1\}$ as $\omega_{T}(f) \neq \varnothing$. Again, by Proposition 4.1.11

$$
\{1\} \subseteq \omega_{T}^{+}\left(f^{-1}\right) \subseteq \sigma\left(f^{-1}\right)=[1,2]
$$

Let $\lambda \in(1,2]$ and suppose that $\lambda \mathbf{1}-f^{-1} \in \mathcal{W}_{T}^{+}$. Then there exists an invertible extension, say, $g$ of $\left(\lambda \mathbf{1}-f^{-1}\right)_{\mid \mathbf{1}(K)}$ to $K$ satisfying $\lambda \mathbf{1}-f^{-1} \geq g$. Moreover, $g(K) \subseteq \mathbb{R}, g\left(\frac{1}{2}\right) \leq\left(\lambda \mathbf{1}-f^{-1}\right)\left(\frac{1}{2}\right)=\lambda-2 \leq 0$ and $g(1)=(\lambda \mathbf{1}-$ $\left.f^{-1}\right)(1)=\lambda-1>0$. The intermediate value theorem yields a contradiction to the invertibility of $g$, so that $\lambda \mathbf{1}-f^{-1} \notin \mathcal{W}_{T}^{+}$. Hence $(1,2] \subseteq \omega_{T}^{+}\left(f^{-1}\right)$, and the result then follows.

We have the following result.

Proposition 4.2.5. Let $(A, C)$ be an $O B A$ and $T: A \rightarrow B$ be a Banach algebra homomorphism which satisfies the Riesz property. If $a \in A^{-1} \cap C$, then

(i) $\left(\omega_{T}^{+}(a)\right)^{-1} \subseteq \omega_{T}^{+}\left(a^{-1}\right)$,

(ii) $\left(\beta_{T}^{+}(a)\right)^{-1} \subseteq \beta_{T}^{+}\left(a^{-1}\right)$.

Moreover, if $C$ is inverse-closed, then we have equalities in (i) and (ii).

Proof. Assume that $a \in A^{-1} \cap C$. Then $0 \notin \omega_{T}^{+}(a)$ and $0 \notin \omega_{T}^{+}\left(a^{-1}\right)$ follow from the fact that $0 \notin \sigma(a) \cup \sigma\left(a^{-1}\right)$ and Proposition 4.1.11. Also, $0 \notin \beta_{T}^{+}(a)$ and $0 \notin \beta_{T}^{+}\left(a^{-1}\right)$.

(i) If $0 \neq \lambda \notin \omega_{T}^{+}\left(a^{-1}\right)$, then $\lambda\left(a-\frac{1}{\lambda} \mathbf{1}\right) a^{-1}=\lambda \mathbf{1}-a^{-1} \in \mathcal{W}_{T}^{+}$, so that $\left(\frac{1}{\lambda} \mathbf{1}-a\right) a^{-1} \in \mathcal{W}_{T}^{+}$by Lemma 3.3.4. Since $a \in A^{-1} \cap C$, it follows that $\frac{1}{\lambda} \mathbf{1}-a \in \mathcal{W}_{T}^{+}$, and therefore $\lambda \notin\left(\omega_{T}^{+}(a)\right)^{-1}$.

(ii) This follows by using the same argument as that in the proof of (i).

For the converse inclusions, suppose now that $C$ is inverse-closed. Again, we give the proof for the upper Weyl spectrum; the proof for the upper Browder spectrum is done in a similar manner. Let $0 \neq \lambda \notin\left(\omega_{T}^{+}(a)\right)^{-1}$. Then $\frac{1}{\lambda} \mathbf{1}-a \in \mathcal{W}_{T}^{+}$, and hence $a-\frac{1}{\lambda} \mathbf{1} \in \mathcal{W}_{T}^{+}$by Lemma 3.3.4. Using the fact that $a^{-1} \in A^{-1} \cap C$, it follows that $\left(a-\frac{1}{\lambda} \mathbf{1}\right) a^{-1} \in \mathcal{W}_{T}^{+}$, and consequently $\lambda \mathbf{1}-a^{-1}=\lambda\left(a-\frac{1}{\lambda} \mathbf{1}\right) a^{-1} \in \mathcal{W}_{T}^{+}$by recalling Lemma 3.3.4 again. This gives $\lambda \notin \omega_{T}^{+}\left(a^{-1}\right)$.

Although Example 4.2.4 illustrates that the assumption about the Riesz property is not in general necessary to obtain (i) and (ii) in the above result, it does show that the condition " $T$ has the Riesz property" in Proposition 
4.2.5 is essential to achieve identities. In general, it appears that, in developing a spectral inclusion theorem for the upper Weyl and upper Browder spectra (dealing with more general holomorphic functions), the assumption that $T$ has the Riesz property may be essential. Even the assumption about the inverse-closedness of the algebra cone may be of importance.

\subsection{Connected hulls}

Throughout this section we let $E$ denote a Banach lattice and $K$ the algebra cone $\left\{T \in \mathcal{L}(E): T E_{+} \subseteq E_{+}\right\}$of positive operators on $E$. We start by recalling the following general terminology and notation. The spectrum of a regular operator $T$ in $\mathcal{L}^{r}(E)$ is called the o-spectrum of $T$ and will be indicated by $\sigma_{o}(T)$. The $o$-spectrum was first investigated by Schaefer [36]. Obviously, the inclusion $\sigma(T) \subseteq \sigma_{o}(T)$ holds. We let $\sigma_{\mathcal{e}}(T):=$ $\sigma(T+\mathcal{K}(E), \mathcal{L}(E) / \mathcal{K}(E))$ denote the essential spectrum of $T \in \mathcal{L}(E)$ and $\sigma_{\text {oe }}(T):=\sigma\left(T+\mathcal{K}^{r}(E), \mathcal{L}^{r}(E) / \mathcal{K}^{r}(E)\right)$ the order essential spectrum of $T \in$ $\mathcal{L}^{r}(E)$. By ([9], Theorem 4.2(a)) we have that $\sigma_{e}(T) \subseteq \sigma_{o e}(T)$.

Bearing in mind Remark 1.1.4 (and the remark in the second paragraph on p.16), recall that the Weyl and upper Weyl spectra relative to $\pi$ (respectively, $\left.\pi_{r}\right)$ coincide [([4], Theorem 16) and this thesis Example 3.1.3]. These are very important examples of homomorphisms with closed range satisfying the Riesz property. We wish to point out the following observation.

Lemma 4.3.1. Let E be a Banach lattice.

(i) If $T \in \mathcal{L}(E)$, then $p(T, \lambda) \in \operatorname{span}(K \cap \mathrm{N}(\pi))$ for all $\lambda \in($ iso $\sigma(T)) \backslash \sigma_{e}(T)$.

(ii) If $T \in \mathcal{L}^{r}(E)$, then $p(T, \lambda) \in \operatorname{span}\left(K \cap \mathrm{N}\left(\pi_{r}\right)\right)$ for all $\lambda \in\left(\right.$ iso $\left.\sigma_{o}(T)\right) \backslash \sigma_{o e}(T)$.

Proof. (i) Let $\lambda \in($ iso $\sigma(T)) \backslash \sigma_{e}(T)$. From ([2], Theorem 7.44(1)) we have that $p(T, \lambda)$ is a finite rank operator on $E$. The result then follows from the inclusions $\overline{\mathcal{F}(E)} \subseteq \mathcal{K}(E)=\mathrm{N}(\pi)$ and $\mathcal{F}(E) \subseteq$ span $(K \cap \overline{\mathcal{F}(E)})$ (see our discussion in Section 3.2).

(ii) Suppose now that $\lambda \in$ (iso $\left.\sigma_{o}(T)\right) \backslash \sigma_{o e}(T)$. In view of ([9], Corollary 3.6) we have that $\lambda \in($ iso $\sigma(T)) \backslash \sigma_{e}(T)$, and hence $p(T, \lambda) \in \mathcal{F}(E)$ by recalling ([2], Theorem 7.44(1)) again. From our discussion in Example 3.1.3 it follows that $\left.\mathcal{F}(E) \subseteq \mathcal{K}^{r}(E)\right)=\operatorname{span}\left(K \cap \mathcal{K}^{r}(E)\right)$, and therefore $p(T, \lambda) \in \operatorname{span}\left(K \cap \mathrm{N}\left(\pi_{r}\right)\right)$. 
Theorem 1.5.8, a result due to Mouton, Mouton and Raubenheimer, states that the connected hulls of the Fredholm, Browder and Weyl spectra (relative to an arbitrary Banach algebra homomorphism with closed range satisfying the Riesz property) coincide. Focussing on elements $a$ of a general OBA $(A, C)$ with the property that $p(a, \lambda) \in \operatorname{span}(C \cap \mathrm{N}(T))$ for all $\lambda \in($ iso $\sigma(a)) \backslash \sigma(T a)$, where $T: A \rightarrow B$ denotes an arbitrary Banach algebra homomorphism with closed range satisfying the Riesz property, we show next that even the connected hull of the upper Weyl spectrum of $a$ can be added to the list in Theorem 1.5.8.

Theorem 4.3.2. Let $(A, C)$ be an $O B A$ and $T: A \rightarrow B$ be a Banach algebra homomorphism with closed range satisfying the Riesz property. If $a \in A$ is such that

$$
p(a, \lambda) \in \operatorname{span}(C \cap \mathrm{N}(T)) \text { for all } \lambda \in(\text { iso } \sigma(a)) \backslash \sigma(T a),
$$

then $\eta \sigma(T a)=\eta \omega_{T}(a)=\eta \beta_{T}(a)=\eta \omega_{T}^{+}(a)$.

Proof. In view of Theorem 1.5.8 we are only left to establish the last identity. Suppose that $a \in A$ is such that $p(a, \lambda) \in \operatorname{span}(C \cap \mathrm{N}(T))$ for all $\lambda \in$ (iso $\sigma(a)) \backslash \sigma(T a)$. Since the inclusion $\subseteq$ is obvious, it is enough to prove the opposite inclusion. We do so by showing that $\sigma(a) \backslash \eta \beta_{T}(a) \subseteq \mathbb{C} \backslash \omega_{T}^{+}(a)$.

Let $\lambda \in \sigma(a) \backslash \eta \beta_{T}(a)$. Then $\lambda \mathbf{1}-a \in \mathcal{B}_{T}=A^{D} \cap \mathcal{F}_{T}$ in light of Proposition 1.5.4, and hence $\lambda \in$ (iso $\sigma(a)) \backslash \sigma(T a)$ by the definition of the almost invertible Fredholm spectrum. Consequently, $p(a, \lambda) \in \operatorname{span}(C \cap \mathrm{N}(T))$ by assumption, which gives $p:=p(\lambda \mathbf{1}-a, 0) \in \operatorname{span}(C \cap \mathrm{N}(T))$ in view of Lemma 1.3.4. Now

$$
\lambda \mathbf{1}-a=\lambda \mathbf{1}-a-p+p=\lambda \mathbf{1}-a-p+\left(\lambda_{1} c_{1}+\lambda_{2} c_{2}+\cdots+\lambda_{n} c_{n}\right)
$$

for some scalars $\lambda_{1}, \ldots, \lambda_{n} \in \mathbb{C}, c_{1}, \ldots, c_{n} \in C \cap \mathrm{N}(T)$ and where $\lambda \mathbf{1}-a-$ $p \in A^{-1}$ follows from Corollary 2.2.6. By Corollary 3.2.3 we have that

$$
\lambda \mathbf{1}-a=(\lambda \mathbf{1}-a-p)_{n}+\mu_{1} c_{1}+\mu_{2} c_{2}+\cdots+\mu_{n} c_{n},
$$

where $\mu_{1}, \ldots, \mu_{n} \geq 0$ and $(\lambda \mathbf{1}-a-p)_{n} \in A^{-1}$, and therefore $\lambda \mathbf{1}-a \in$ $A^{-1}+(C \cap \mathrm{N}(T))=\mathcal{W}_{T}^{+}$; that is $\lambda \notin \omega_{T}^{+}(a)$. We have thus shown that the inclusion $\sigma(a) \backslash \eta \beta_{T}(a) \subseteq \mathbb{C} \backslash \omega_{T}^{+}(a)$ holds, and hence $\omega_{T}^{+}(a) \subseteq \eta \beta_{T}(a)$, so that $\eta \omega_{T}^{+}(a) \subseteq \eta \eta \beta_{T}(a)=\eta \beta_{T}(a)$.

Some remarks: Recall that $p(a, \lambda) \in \mathrm{N}(T)$ for all $\lambda \in($ iso $\sigma(a)) \backslash \sigma(T a)$ (Lemma 2.2.7). Under the stronger assumption " $p(a, \lambda) \in \operatorname{span}(C \cap \mathrm{N}(T))$ 
for all $\lambda \in$ (iso $\sigma(a)) \backslash \sigma(T a)$ ", we have from Theorem 4.3.2 that the identity $\eta \omega_{T}(a)=\eta \omega_{T}^{+}(a)$ holds. If this assumption is further strengthened to $\mathrm{N}(T)=\operatorname{span}(C \cap \mathrm{N}(T))$, then by Theorem 3.2.6(a) the even stronger conclusion $\omega_{T}(a)=\omega_{T}^{+}(a)$ is obtained.

We point out that, at this stage, we do not know (besides in the operator case) whether the statement " $p(a, \lambda) \in \operatorname{span}(C \cap \mathrm{N}(T))$ " in Theorem 4.3.2 is true for all elements $a$ such that $\lambda \in($ iso $\sigma(a)) \backslash \sigma(T a)$.

In the following example we demonstrate that the upper Browder spectrum cannot generally be added to the list of identities in Theorem 4.3.2.

Example 4.3.3. Consider the Banach algebra homomorphism $T: M_{3}^{u}(\mathbb{C}) \rightarrow \mathbb{C}$ defined by

$$
T\left[\begin{array}{ccc}
x_{11} & x_{12} & x_{13} \\
0 & x_{22} & x_{23} \\
0 & 0 & x_{33}
\end{array}\right]=x_{11}
$$

and $M:=\left(\begin{array}{lll}1 & 1 & 0 \\ 0 & 0 & 1 \\ 0 & 0 & 0\end{array}\right) \in M_{3}^{u}(\mathbb{C})$. Then $\eta \omega_{T}^{+}(M) \neq \eta \beta_{T}^{+}(M)$.

The homomorphism $T$ has closed range (in fact it is onto) and satisfies the Riesz property. Also, the identity $\mathrm{N}(T)=\operatorname{span}\left(M_{3}^{u}\left(\mathbb{R}^{+}\right) \cap \mathrm{N}(T)\right)$ was established in Example 3.2.12. By recalling Example 4.1.14 we have that $\eta \omega_{T}^{+}(M)=\{1\} \neq\{0,1\}=\eta \beta_{T}^{+}(M)$. 


\section{Chapter 5}

\section{The upper Browder spectrum property}

In this chapter we continue our discussion on the upper Browder spectrum introduced in Chapter 4. Our main purpose here is to investigate, in an arbitrary OBA, certain questions that originated in the context of bounded linear operators on Banach lattices and appeared in the papers [3] and [4] by Alekhno. It is assumed throughout that $E$ denotes a Banach lattice and $\pi: \mathcal{L}(E) \rightarrow \mathcal{L}(E) / \mathcal{K}(E)$ the canonical homomorphism on $\mathcal{L}(E)$.

\subsection{Introduction}

If $T \in \mathcal{L}(E)$, then the set of inclusions

$$
\sigma_{e}(T) \subseteq \omega_{\pi}(T) \subseteq \omega_{\pi}^{+}(T) \subseteq \sigma(T)
$$

were established by Alekhno in ([3], p.376). It is known that, if the spectral radius $r(T)$ of $T$ is an element of $\sigma(T)$ (this is always the case for positive operators by ([35], Proposition 4.1, p.323)) but not of $\sigma_{e}(T)$, then $r(T)$ is also not in $\omega_{\pi}(T)$. This can be seen in the following two ways:

(i) Due to the nature of the element $r(T)$, the assumption $r(T) \notin \sigma_{e}(T)$ is equivalent to $r(T) \notin \eta \sigma_{e}(T)$. Using the identity

$$
\eta \sigma_{e}(T)=\eta \omega_{\pi}(T)=\eta \beta_{\pi}(T)
$$

(see Theorem 1.5.8), it follows that the implication $r(T) \notin \sigma_{e}(T) \Rightarrow r(T) \notin$ $\omega_{\pi}(T)$ holds. 
(ii) If $r(T) \notin \sigma_{e}(T)$, then $r(T)$ is a pole of $(\lambda I-T)^{-1}$ by Corollary 2.1.5. From Corollary 2.2.6 we have that $r(T) \notin \sigma\left(T+T_{-1}\right)$, where $T_{-1}=P(T, r(T))$ is the spectral idempotent of $T$ corresponding to $r(T)$. Using the fact that $T_{-1} \in \mathcal{F}(E) \subseteq \mathcal{K}(E)$ we have that $r(T) \notin \bigcap_{K \in \mathcal{K}(E)} \sigma(T+K)=\omega_{\pi}(T)$.

A natural question which arises from (5.1.1) is the following: given it is known that the spectral radius of $T$ is an element of $\sigma(T)$ but not of $\sigma_{e}(T)$, can we conclude that

$$
r(T) \notin \omega_{\pi}^{+}(T) ?
$$

In his 2007-paper [3] Alekhno investigated the aforementioned question. However, in his subsequent article, he found that there is always the implication

$$
r(T) \notin \sigma_{e}(T) \Rightarrow r(T) \notin \omega_{\pi}^{+}(T)
$$

as the equality $\omega_{\pi}(T)=\omega_{\pi}^{+}(T)$ holds (see [4], Theorem 16).

Recall that, in Chapter 4, we introduced the upper Weyl spectrum of an element of an arbitrary OBA and established the inclusions

$$
\sigma(T a) \subseteq \omega_{T}(a) \subseteq \omega_{T}^{+}(a) \subseteq \sigma(a)
$$

in Proposition 4.1.11, where $T$ denotes a homomorphism from an $\operatorname{OBA}(A, C)$ to an arbitrary Banach algebra and $a \in A$. In view of Theorem 4.3.2 we can state the following result.

Proposition 5.1.5. Let $(A, C)$ be an $O B A$ and $T: A \rightarrow B$ be a Banach algebra homomorphism with closed range satisfying the Riesz property. If $a \in A$ is such that $p(a, \lambda) \in \operatorname{span}(C \cap \mathrm{N}(T))$ for all $\lambda \in($ iso $\sigma(a)) \backslash \sigma(T a)$, then $r(a) \notin \sigma(T a)$ implies that $r(a) \notin \omega_{T}^{+}(a)$.

A quick glance at the proof of Theorem 4.3.2 (or, instead, Corollary 2.2.6 together with the representation of the upper Weyl spectrum provided in Corollary 4.1.10) reveals that Proposition 5.1.5 remains valid if the statement " $p(a, \lambda) \in \operatorname{span}(C \cap \mathrm{N}(T))$ for all $\lambda \in($ iso $\sigma(a)) \backslash \sigma(T a)$ " is true only for $\lambda=r(a)$.

In this thesis (see Definition 4.1.1) the concept of the upper Browder spectrum of an element in an OBA was also introduced and has not been studied in the context of bounded linear operators on Banach lattices. From Proposition 4.1.11 we have that $\sigma(T a) \subseteq \omega_{T}^{+}(a) \subseteq \beta_{T}^{+}(a) \subseteq \sigma(a)$, where $T$ denotes 
a homomorphism from an $\mathrm{OBA}(A, C)$ to an arbitrary Banach algebra and $a \in A$.

In view of Proposition 5.1 .5 (together with the observation thereafter) and Example 4.3.3, we are interested in investigating the following more general question: if the spectral radius of an OBA element is outside its Fredholm spectrum, what conditions suffice for it to be outside the upper Browder spectrum of the element? Of course we are only interested in OBA elements $a$ with the property that $r(a) \in \sigma(a)$. Consequently, as a result of Theorem 1.8.2, our study will focus on positive elements.

Definition 5.1.6 (Upper Browder spectrum property). Let $(A, C)$ be an $O B A$ and $T: A \rightarrow B$ be a Banach algebra homomorphism. Then $a \in C$ is said to have the upper Browder spectrum property (relative to $T$ ) if there is the implication $r(a) \notin \sigma(T a) \Rightarrow r(a) \notin \beta_{T}^{+}(a)$.

As a starting point, we recall the following result by Alekhno.

Theorem 5.1.7. ([4], Theorem 18(a)) Let E be a Dedekind complete Banach lattice such that the order continuous dual $E_{n}^{\sim}$ separates the points of $E$. If $T \geq 0$ is an order continuous operator on $E$ such that $r(T) \notin \sigma_{e}(T)$, then $r(T) \notin \sigma(T+$ $\alpha\left|T_{-1}\right|$ ) for all $0 \neq \alpha \in \mathbb{C}$.

Some remarks: Since $T_{-1} \in \mathcal{F}(E)$, we have from ([7], Theorem 16.8) that $\left|T_{-1}\right|$ exists and $\left|T_{-1}\right| \in \mathcal{K}(E)$, and hence $\alpha\left|T_{-1}\right|$ is a positive compact operator for all $\alpha \in \mathbb{R}^{+}$. However, since $a a_{-1}=a_{-1} a$, where $a$ is an arbitrary OBA element satisfying $r(a) \in$ iso $\sigma(a)$ and $a_{-1}:=p(a, r(a))$, does not in general imply that $a\left|a_{-1}\right|=\left|a_{-1}\right| a$, we cannot deduce from Theorem 5.1.7 that $r(T) \notin \beta_{\pi}^{+}(T)$.

At this moment, it is still unclear whether all positive operators on an arbitrary Banach lattice have the upper Browder spectrum property. We doubt this is the case, although at present we do not have a counterexample. In 2012 Alekhno presented the following analogue of Theorem 5.1.7 in the OBA setting.

Theorem 5.1.8. ([6], Theorem 5.5) Let $(A, C)$ be a Dedekind complete OBA which has a disjunctive product with closed and proper algebra cone $C$. If $a \in C$ is a spectrally order continuous element such that $r(a)$ is an $f$-pole of $(\lambda \mathbf{1}-a)^{-1}$ and $\left|a_{-1}\right|$ exists, then $r(a) \notin \sigma\left(a+\alpha\left|a_{-1}\right|\right)$ for all $0 \neq \alpha \in \mathbb{C}$. 
It is worth noting that, unlike in the operator case, the element $\left|a_{-1}\right|$ does not necessarily exist.

We will continue our discussion on Dedekind complete OBAs with disjunctive products in Section 5.5.

The main purpose of this chapter is to display different types of sufficient conditions for positive OBA elements to have the upper Browder spectrum property. We point out that, in view of Proposition 5.1.5, we shall be concerned with homomorphisms with closed range having the Riesz property.

\subsection{Finite-dimensional semisimple OBAs}

First we consider a special class of Banach algebra homomorphisms with closed range satisfying the Riesz property: homomorphisms defined on a finite-dimensional (semisimple) OBA. Since the study of finite-dimensional semisimple (ordered Banach) algebras lead naturally to the WedderburnArtin Theorem, we shall examine homomorphisms from a direct sum of matrix algebras over $\mathbb{C}$ to an arbitrary Banach algebra. Our main result in this section is Corollary 5.2.11. This result tells us that all positive elements of a finite-dimensional semisimple OBA have the upper Browder spectrum property.

We start our discussion with several observations.

Lemma 5.2.1. Suppose that $T: A \rightarrow B$ is a Banach algebra homomorphism with $\operatorname{dim} A<\infty$. Then $\sigma(T a)=\omega_{T}(a)=\beta_{T}(a)$ for all $a \in A$.

Proof. Since $\operatorname{dim} A<\infty$, we have that $\sigma(a)$ is finite for all $a \in A$, and hence $T$ has the Riesz property. It also follows from Corollary 1.1.7 that $T$ has closed range. The result then follows from Theorem 1.5.8 and the fact that the relevant spectra are finite.

By a simple algebra we mean an algebra which has no ideals besides the zero ideal.

Lemma 5.2.2. Let $A$ be a simple Banach algebra and $T: A \rightarrow B$ be a Banach algebra homomorphism with closed range. Then $\eta \sigma(T a)=\eta \sigma(a)$ for all $a \in A$.

Morover, if $A$ is also finite-dimensional, then $\sigma(a)=\sigma(T a)$ for all $a \in A$. (For this case T automatically has closed range.) 
Proof. Since $\mathrm{N}(T)$ is an ideal of a simple Banach algebra $A$, it follows that $\mathrm{N}(T)=\{0\}$, and hence $T$ satisfies the Riesz property. We also have that the identity $\mathcal{B}_{T}=A^{-1}$ holds. Therefore, by using Theorem 1.5.8, the equalities $\eta \sigma(a)=\eta \beta_{T}(a)=\eta \sigma(\mathrm{Ta})$ for all $a \in A$ are clear.

The last statement follows from the fact that the spectra of elements in a finite-dimensional Banach algebra are finite.

In the results which follow, let $0_{A}$ denote the zero element of $A$. The following fact will be needed in Propositions 5.2.4 and 5.2.7.

Lemma 5.2.3. For $t \in \mathbb{N}$, let $A_{1}, \ldots, A_{t}$ be finite-dimensional simple Banach algebras and $T: A_{1} \oplus \cdots \oplus A_{t} \rightarrow B$ be a Banach algebra homomorphism. For each $j \in\{1, \ldots, t\}$, let $A_{j}^{\prime}:=\left\{0_{A_{1}}\right\} \oplus \cdots \oplus A_{j} \oplus \cdots \oplus\left\{0_{A_{t}}\right\}$ and define $T_{j}: A_{j}^{\prime} \rightarrow B$ by $T_{j}\left(0_{A_{1}}, \ldots, a_{j}, \ldots, 0_{A_{t}}\right)=T\left(0_{A_{1}}, \ldots, a_{j}, \ldots, 0_{A_{t}}\right)$ where $a_{j} \in A_{j}$. Then either $T_{j}=0$ or $T_{j}\left(A_{j}^{\prime}\right)$ is a Banach algebra and $T_{j}: A_{j}^{\prime} \rightarrow T_{j}\left(A_{j}^{\prime}\right)$ is an isomorphism.

Proof. Let $j \in\{1, \ldots, n\}$. Since $A_{j}^{\prime}$ is isomorphic to $A_{j}$, we have that $A_{j}^{\prime}$ is a finite-dimensional simple Banach algebra. Using the fact that $T$ is a Banach algebra homomorphism, we have that $T_{j}$ is a linear operator (not necessarily non-zero) which preserves multiplication. For the rest of the proof, consider a non-zero $T_{j}$. (This is possible as $T \neq 0$.) Then $\mathrm{N}\left(T_{j}\right)$ is an ideal of $A_{j}^{\prime}$, and hence $\mathrm{N}\left(T_{j}\right)=\left\{0_{A_{1}}\right\} \oplus \cdots \oplus\left\{0_{A_{t}}\right\}$. From Theorems 1.1.5 and 1.1.6 it follows that $T_{j}\left(A_{j}^{\prime}\right)$ is a closed subalgebra of $B$ (and therefore a Banach algebra) with unit $T\left(0_{A_{1}}, \ldots, \mathbf{1}_{A_{j}}, \ldots, 0_{A_{t}}\right)$. Hence $T_{j}: A_{j}^{\prime} \rightarrow T_{j}\left(A_{j}^{\prime}\right)$ is a Banach algebra homomorphism which is one-to-one and onto.

To avoid tedious repetition of notation, it is understood that all notations introduced in Lemma 5.2.3 are still valid in the results that follow.

Proposition 5.2.4. For $t \in \mathbb{N}$, let $A_{1}, \ldots, A_{t}$ be finite-dimensional simple Banach algebras and $T: A_{1} \oplus \cdots \oplus A_{t} \rightarrow B$ be a Banach algebra homomorphism such that all $T_{j}$ 's are non-zero. Then $\sigma(T a)=\sigma(a)$ for all $a \in A_{1} \oplus \cdots \oplus A_{t}$.

Proof. Since the result for $t=1$ is clear from Lemma 5.2.2, we will assume here that $t \geq 2$. By assumption and Lemma 5.2 .3 we have that all $T_{j}: A_{j}^{\prime} \rightarrow T_{j}\left(A_{j}^{\prime}\right)$ are isomorphisms. Since, for each $j \in\{1, \ldots, t\}$, the Banach algebra $A_{j}$ is simple, its only ideal is $\left\{0_{A_{j}}\right\}$. It is not difficult to verify that $A:=A_{1} \oplus \cdots \oplus A_{t}$ has $2^{t}-1$ ideals, each of the form $I_{1} \oplus \cdots \oplus I_{t}$, where $I_{j} \in\left\{\left\{0_{A_{j}}\right\}, A_{j}\right\}$ for all $j \in\{1, \ldots, t\}$. (By the definition of an ideal $A$ is excluded.) 
Since $\mathrm{N}(T)$ is an ideal of $A$, it will take on the form of one of the $2^{t}-1$ ideals of $A$. Via a process of elimination, we determine $\mathrm{N}(T)$.

If $t=2$, then we note that $\mathrm{N}(T)$ cannot coincide with some $A_{j}^{\prime}$ as this would imply that $T_{j}=0$; a contradiction. For this case we have that $\mathrm{N}(T)=$ $\left\{0_{A_{1}}\right\} \oplus\left\{0_{A_{2}}\right\}$.

Suppose now that $t \geq 3$. As before, $\mathrm{N}(T)$ cannot coincide with some $A_{j}^{\prime}$ as this would indicate that $T_{j}=0$; a contradiction. If $\mathrm{N}(T)$ is an ideal which has at least two non-zero components and at least one zero component, then we can find $j \in\{1, \ldots, t\}$ such that $A_{j}^{\prime} \subseteq \mathrm{N}(T)$. Again, this implies that $T_{j}=0$, which is not possible. Therefore $\mathrm{N}(T)=\left\{0_{A_{1}}\right\} \oplus \cdots \oplus\left\{0_{A_{t}}\right\}$.

By Corollary 1.1.7 we have that $T(A)$ is a Banach algebra which contains the unit of $B$. Using Theorem 1.2.2(ii) and the fact that $T: A \rightarrow T(A)$ is an isomorphism, we get that $\sigma(a)=\sigma(T a, T(A))=\sigma(T a, B)$ for all $a \in A$.

Proposition 5.2.4 (in a sense) extends Lemma 5.2.2 to direct sums of finite-dimensional simple Banach algebras.

From ([10], Theorem 2.1.1) we have that $M_{n}(\mathbb{C})(n \geq 1)$ is an example of a finite-dimensional simple Banach algebra. The following example indicates that the assumption in Proposition 5.2.4 that all the $T_{j}$ 's must be non-zero is essential.

Example 5.2.5. Consider the homomorphism $T: M_{2}(\mathbb{C}) \oplus \mathbb{C} \rightarrow \mathbb{C}$ defined by $T(X, \lambda)=\lambda$ for $X \in M_{2}(\mathbb{C})$ and $\lambda \in \mathbb{C}$. Also consider the element $A:=$ $\left(\left(\begin{array}{ll}0 & 0 \\ 0 & 2\end{array}\right), 1\right)$. Then $\sigma(T A)=\{1\} \neq\{0,1,2\}=\sigma(A)$.

Observe from Example 5.2.5 that $T_{1}: M_{2}(\mathbb{C}) \oplus\{0\} \rightarrow \mathbb{C}$ defined by $T_{1}(X, 0)=T(X, 0)$ for $X \in M_{2}(\mathbb{C})$ is the zero map.

The following result is an immediate consequence of Proposition 5.2.4.

Corollary 5.2.6. Let $n_{1}, n_{2}, \ldots, n_{t} \in \mathbb{N}$ for some $t \in \mathbb{N}$ and $A_{j}=M_{n_{j}}(\mathbb{C})$ for all $j \in\{1, \ldots, t\}$.If $T: A_{1} \oplus \cdots \oplus A_{t} \rightarrow B$ denotes a Banach algebra homomorphism with all the $T_{j}$ 's non-zero, then $\sigma(T X)=\sigma(X)$ for all $X \in A_{1} \oplus \cdots \oplus A_{t}$.

In our next result we investigate the case where some of the $T_{j}$ 's might be zero and are concerned with Banach algebra homomorphisms on direct sums of matrix algebras over $\mathbb{C}$.

Proposition 5.2.7. Let $n_{1}, n_{2}, \ldots, n_{t} \in \mathbb{N}$ for some $t \in \mathbb{N}$ and $A_{j}=M_{n_{j}}(\mathbb{C})$ for all $j \in\{1, \ldots, t\}$. Suppose that $T: A_{1} \oplus \cdots \oplus A_{t} \rightarrow B$ is a Banach algebra 
homomorphism with at least one $T_{j}$ zero. Then $\sigma(T X)=\omega_{T}^{+}(X)$ for all $X \in$ $A_{1} \oplus \cdots \oplus A_{t}$.

Furthermore, if $X \in A_{1} \oplus \cdots \oplus A_{t}$ satisfies $r(X) \in \sigma(X) \backslash \sigma(T X)$, then $r(X) \notin \beta_{T}^{+}(X)$.

Proof. Let $A:=A_{1} \oplus \cdots \oplus A_{t}$ and assume that $T_{j}=0$ for all $j \in\left\{s_{1}, \ldots, s_{k}\right\} \subseteq$ $\{1, \ldots, t\}$. From Lemma 5.2.3 we have that $T_{j}: A_{j}^{\prime} \rightarrow T_{j}\left(A_{j}^{\prime}\right)$ is an isomorphism for all $j \in\{1, \ldots, t\} \backslash\left\{s_{1}, \ldots, s_{k}\right\}:=D$.

Again, since $A_{j}$ is simple for all $j \in\{1, \ldots, t\}$, we have that $A$ has $2^{t}-1$ ideals, each of the form $I_{1} \oplus \cdots \oplus I_{t}$, where $I_{j} \in\left\{\left\{0_{A_{j}}\right\}, A_{j}\right\}$ for all $j \in$ $\{1, \ldots, t\}$.

It is easy to verify that $I_{1} \oplus \cdots \oplus I_{t}$, where $I_{j}=A_{j}$ for $j \in\left\{s_{1}, \ldots, s_{k}\right\}$ and $I_{j}=\left\{0_{A_{j}}\right\}$ for $j \in D$, is contained in $\mathrm{N}(T)$. Using a similar reasoning as in the proof of Proposition 5.2.4 we conclude that this subalgebra is precisely the null space of $T$. Therefore, since each $X_{j} \in A_{j}$ can be decomposed as $X_{j}=X_{j_{1}}-X_{j_{2}}+i X_{j_{3}}-i X_{j_{4}}$ with $X_{j_{1}}, X_{j_{2}}, X_{j_{3}}, X_{j_{4}} \in M_{n_{j}}\left(\mathbb{R}^{+}\right)$, it follows that $\mathrm{N}(T)=\operatorname{span}(C \cap \mathrm{N}(T))$, where $C=M_{n_{1}}\left(\mathbb{R}^{+}\right) \oplus \cdots \oplus M_{n_{t}}\left(\mathbb{R}^{+}\right)$. Applying Theorem 3.2.6(a) and Lemma 5.2.1 we have that

$$
\sigma(T X)=\beta_{T}(X)=\omega_{T}(X)=\omega_{T}^{+}(X)
$$

for all $X \in A$, which proves the first part of the proposition.

Now suppose that $X \in A$ is such that $r(X) \in \sigma(X) \backslash \sigma(T X)$. Since $X=$ $\left(X_{1}, \ldots, X_{t}\right)$ with $X_{j} \in A_{j}$, we let $X_{j}^{0}:=\left(\mathbf{0}_{A_{1}}, \ldots, X_{j}, \ldots, \mathbf{0}_{A_{t}}\right)$, where $X_{j}$ is in the $j$-th position of the $n_{t}$-tuple. Consequently,

$$
X=\left(X_{1}, \ldots, \mathbf{0}_{A_{t}}\right)+\cdots+\left(\mathbf{0}_{A_{1}}, \ldots, X_{t}\right)=X_{1}^{0}+\cdots+X_{t}^{0}
$$

with $X_{i}^{0} X_{j}^{0}=0=X_{j}^{0} X_{i}^{0}$ for all $i \neq j$. By using ([10], Exercise 9, p.66) and the fact that $\sigma(Y, A)$ (and therefore $\sigma(T Y, B)$ ) is finite for all $Y \in A$, it then follows that

$$
\begin{aligned}
\sigma^{\prime}(T X, B) & =\sigma^{\prime}\left(T\left(X_{1}^{0}+\cdots+X_{t}^{0}\right), B\right) \\
& =\sigma^{\prime}\left(T X_{1}^{0}+\cdots+T X_{t}^{0}, B\right) \\
& =\left(\sigma\left(T X_{1}^{0}, B\right) \cup \cdots \cup \sigma\left(T X_{t}^{0}, B\right)\right) \backslash\{0\} \\
& =\sigma^{\prime}\left(T X_{1}^{0}, B\right) \cup \cdots \cup \sigma^{\prime}\left(T X_{t}^{0}, B\right) \\
& =\sigma^{\prime}\left(T_{1} X_{1}^{0}, B\right) \cup \cdots \cup \sigma^{\prime}\left(T_{t} X_{t}^{0}, B\right) \\
& =\bigcup_{j \in D} \sigma^{\prime}\left(T_{j} X_{j}^{0}, B\right) .
\end{aligned}
$$


Since, for $j \in D$, the range $T_{j}\left(A_{j}^{\prime}\right)$ of $T_{j}$ is a closed subalgebra of the Banach algebra $B$, we have from Theorem 1.2.5 and the remark following Proposition 1.2.3 that $\sigma^{\prime}\left(T_{j} X_{j}^{0}, T_{j}\left(A_{j}^{\prime}\right)\right)=\sigma^{\prime}\left(T_{j} X_{j}^{0}, B\right)$. Hence, using (5.2.8) and the facts that $T_{j}: A_{j}^{\prime} \rightarrow T_{j}\left(A_{j}^{\prime}\right)$ is an isomorphism and $A_{j}^{\prime}$ is isomorphic to $A_{j}$, it follows that

$$
\begin{aligned}
\sigma^{\prime}(T X, B) & =\bigcup_{j \in D} \sigma^{\prime}\left(T_{j} X_{j}^{0}, B\right) \\
& =\bigcup_{j \in D} \sigma^{\prime}\left(T_{j} X_{j}^{0}, T_{j}\left(A_{j}^{\prime}\right)\right) \\
& =\left(\bigcup_{j \in D} \sigma\left(T_{j} X_{j}^{0}, T_{j}\left(A_{j}^{\prime}\right)\right)\right) \backslash\{0\} \\
& =\left(\bigcup_{j \in D} \sigma\left(X_{j}^{0}, A_{j}^{\prime}\right)\right) \backslash\{0\} \\
& =\left(\bigcup_{j \in D} \sigma\left(X_{j}, A_{j}\right)\right) \backslash\{0\} .
\end{aligned}
$$

Let $\lambda$ be a positive real scalar satisfying $\lambda>r(X)\left(\geq r\left(X_{j}\right)\right)$ and consider the decomposition

$$
X-r(X) \mathbf{1}_{A}=\left(X_{1}-r(X) \mathbf{1}_{A_{1}}, \ldots, X_{t}-r(X) \mathbf{1}_{A_{t}}\right)=\left(U_{1}, \ldots, U_{t}\right)+\left(V_{1}, \ldots, V_{t}\right),
$$

where the $U_{j}$ and $V_{j}$ are chosen as follows: If $j \in D$, then $r(X) \notin \sigma\left(X_{j}\right)$, so choose $U_{j}=X_{j}-r(X) \mathbf{1}_{A_{j}} \in A_{j}^{-1}$ and $V_{j}=0_{A_{j}}$. If $j \notin D$, then choose $U_{j}=X_{j}-\lambda \mathbf{1}_{A_{j}} \in A_{j}^{-1}$ and $V_{j}=(\lambda-r(X)) \mathbf{1}_{A_{j}}$.

In both cases the $U_{j}$ 's are invertible in $A_{j}$, and hence $\left(U_{1}, \ldots, U_{t}\right) \in A^{-1}$. We are only left to verify whether our selected $\left(V_{1}, \ldots, V_{t}\right)$ is an element of $C \cap \mathrm{N}(T)$. Recall that $\mathrm{N}(T)=I_{1} \oplus \cdots \oplus I_{t}$, where $I_{j}=A_{j}$ for $j \notin D$ and $I_{j}=\left\{0_{A_{j}}\right\}$ for $j \in D$. Since

$$
V_{j}=\left\{\begin{aligned}
(\lambda-r(X)) \mathbf{1}_{A_{j}} \in C_{j} \cap A_{j} & \text { if } j \notin D \\
0_{A_{j}} \in C_{j} \cap\left\{0_{A_{j}}\right\} & \text { if } j \in D,
\end{aligned}\right.
$$

it follows that $\left(V_{1}, \ldots, V_{t}\right) \in C \cap \mathrm{N}(T)$, and hence $X-r(X) \mathbf{1}_{A} \in \mathcal{B}_{T}^{+}$. By Proposition 4.1.3 $r(X) \notin \beta_{T}^{+}(X)$.

Remark 5.2.9. We mention that, more generally, the first part of Proposition 5.2.7 is true whenever each $A_{j}$ is a finite-dimensional simple $O B A$ with generating cone, while the last part holds for each $A_{j}$ an arbitrary finite-dimensional simple $O B A$. 
The observations in Corollary 5.2.6 and Proposition 5.2.7 lead directly to the following result.

Theorem 5.2.10. Any finite-dimensional semisimple $O B A$ is algebraically isomorphic to an $\operatorname{OBA}(A, C)$ with the property that, if $T: A \rightarrow B$ is a Banach algebra homomorphism and $a \in A$ is such that $r(a) \in \sigma(a)$ and $r(a) \notin \sigma(T a)$, then $r(a) \notin \beta_{T}^{+}(a)$.

Proof. By the Wedderburn-Artin Theorem ([10], Theorem 2.1.2) we can take $A=M_{n_{1}}(\mathbb{C}) \oplus M_{n_{2}}(\mathbb{C}) \oplus \cdots \oplus M_{n_{k}}(\mathbb{C})$, with $n_{1}, n_{2}, \ldots, n_{k} \in \mathbb{N}$. We choose $C=M_{n_{1}}\left(\mathbb{R}^{+}\right) \oplus M_{n_{2}}\left(\mathbb{R}^{+}\right) \oplus \cdots \oplus M_{n_{k}}\left(\mathbb{R}^{+}\right)$. Note that our assumption here indicates that the use of Corollary 5.2.6 is ruled out. Nonetheless, the desired result follows from the second part of Proposition 5.2.7.

Corollary 5.2.11. Any finite-dimensional semisimple $O B A$ is algebraically isomorphic to an $\operatorname{OBA}(A, C)$ with the property that all positive elements in $A$ have the upper Browder spectrum property relative to arbitrary Banach algebra homomorphisms $T: A \rightarrow B$.

Proof. Choose $A$ and $C$ as in the proof of Theorem 5.2.10. Since each $M_{n_{i}}\left(\mathbb{R}^{+}\right)$ is a closed and normal algebra cone in $M_{n_{i}}(\mathbb{C})$, it follows from the remark preceding Definition 1.7.2 that $C$ is a closed and normal algebra cone in $A$. Hence, in view of Theorem 1.8.2, we have that $r(a) \in \sigma(a)$ whenever $a \in C$. The result then follows from Theorem 5.2.10.

\subsection{Homomorphisms with closed range having the Riesz property}

We provide here sufficient conditions for positive OBA elements to have the upper Browder spectrum property relative to arbitrary Banach algebra homomorphisms with closed range satisfying the Riesz property. Our main results in this section include Theorem 5.3.3 and Proposition 5.3.5.

We shall begin with a simple observation which forms the basis of our contributions in this section.

Theorem 5.3.1. Let $(A, C)$ be an $O B A$ and $I$ be an ideal of $A$. Suppose that $a \in A$ is such that $r(a)$ is a Riesz point of $\sigma(a)$ relative to I. If $p(a, r(a)) \in C$, then there exists $c \in C \cap I \cap \operatorname{Comm}(a)$ satisfying $r(a) \notin \sigma(a+c)$. 
Proof. Let $a \in A$ be such that $r(a)$ is a Riesz point of $\sigma(a)$ relative to $I$. By definition, $r(a) \in$ iso $\sigma(a)$ and $p(a, r(a)) \in I$, so that $a_{-1} \in C \cap I \cap \operatorname{Comm}(a)$ follows from assumption. The result then follows from Corollary 2.2.6, with $c=a_{-1}$.

Theorem 5.3.1 was proved by Alekhno [see [3], Theorem 4(a)] in the operator algebra $\mathcal{L}(E)$ with $\mathcal{F}(E)$ the ideal of finite-rank operators on $E$. We point out that, the proof given here uses the spectral mapping theorem, while Alekhno's proof is based on operator theory techniques.

The following result is a consequence of Theorem 5.3.1.

Corollary 5.3.2. Let $(A, C)$ be an $O B A$ with closed algebra cone $C$ and let $I$ be an inessential ideal of $A$. If $a \in C$ is such that $r(a)$ is a Riesz point of $\sigma(a)$ relative to $I$, then, under each of the following assumptions, there exists $c \in C \cap I \cap \operatorname{Comm}(a)$ such that $r(a) \notin \sigma(a+c)$ :

(i) $r(a)$ is a simple pole of $(\lambda \mathbf{1}-a)^{-1}$,

(ii) $A$ is commutative and semisimple,

(iii) $A$ is semisimple and $C$ is proper and inverse-closed.

Proof. Let $a \in C$ be such that $r(a)$ is a Riesz point of $\sigma(a)$ relative to $I$.

(i) This is clear from Theorems 1.8.1 and 5.3.1.

(ii) Suppose that $A$ is commutative and semisimple. From Lemma 1.4.5 we have that $r(a)$ is a pole of $(\lambda \mathbf{1}-a)^{-1}$, and hence a simple pole by Lemma 2.2.8. The result follows from (i).

(iii) Suppose that $A$ is semisimple with proper and inverse-closed algebra cone $C$. The proof is similar to the proof of (ii): just replace Lemma 2.2.8 with Lemma 2.2.9.

We are now ready to state our first result in this section for Banach algebra homomorphisms with closed range satisfying the Riesz property. We mention that (in view of Theorem 1.8.2 and Corollary 2.1.5), in the following result, $r(a)$ is a Riesz point of $\sigma(a)$ relative to $\mathrm{N}(T)$, and therefore $p(a, r(a))$ exists.

Theorem 5.3.3. Let $(A, C)$ be an $O B A$ with closed algebra cone $C$ such that the spectral radius function in $(A, C)$ is monotone. Suppose that $T: A \rightarrow B$ is a Banach algebra homomorphism with closed range satisfying the Riesz property and $a \in C$ is such that $r(a) \notin \sigma($ Ta $)$. If $p(a, r(a)) \in C$, then $r(a) \notin \beta_{T}^{+}(a)$. 
Hence, each of the following conditions ensures that $r(a) \notin \beta_{T}^{+}(a)$ :

(i) $r(a)$ is a simple pole of $(\lambda \mathbf{1}-a)^{-1}$,

(ii) $A$ is commutative and semisimple,

(iii) $A$ is semisimple and $C$ is proper and inverse-closed.

Proof. Suppose that $p(a, r(a)) \in C$. By Theorem 5.3.1 we can find an element $c \in C \cap \mathrm{N}(T) \cap \operatorname{Comm}(a)$ such that $r(a) \notin \sigma(a+c)$, and hence $r(a) \notin \bigcap_{\substack{c \in C \cap N(T) \\ a c=c a}} \sigma(a+c)=\beta_{T}^{+}(a)$ using (4.1.5).

For the second part of the theorem, we observe from the proof of Corollary 5.3.2 that, if any of the statements (i) - (iii) hold, then $p(a, r(a)) \in C$. Hence the result follows from the first part of the theorem.

Since the canonical homomorphism $T: A \rightarrow A / \bar{I}$, where $(A, C)$ denotes an OBA and $I$ an inessential ideal of $A$, has closed range and satisfies the Riesz property, the preceding theorem can be applied to $T$.

For the purpose of our next example we establish the following notation. By $Z(E)$ we denote the center of a Banach lattice $E$, given by $Z(E):=\{T \in \mathcal{L}(E):$ there exists $c>0$ such that $|T x| \leq c|x|$ for all $x \in E\}$, which is a Banach algebra according to ([2], Theorem 3.31). As an application of Theorem 5.3.3 we have the following:

Example 5.3.4. If $T \in Z(E)$ is a positive operator such that $r(T) \notin \sigma_{e}(T)$, then $r(T) \notin \beta_{\pi}^{+}(T)$.

By ([9], Theorem 8.2(a)) we have that $P(T, r(T)) \in \mathbf{O I}(\mathcal{L}(E))$. Since $P(T, r(T))$ is a positive operator, Theorem 5.3.3 is applicable.

Also, in view of ([2], Theorems 3.6 and 3.31(1)), $Z(E)=C(K)$ for some compact Hausdorff space $K$. Hence $Z(E)$ is an example of a commutative and semisimple OBA, so that by Theorem 5.3 .3 all positive operators in $Z(E)$ have the upper Browder spectrum property relative to arbitrary Banach algebra homomorphisms (acting on $Z(E)$ ) with closed range satisfying the Riesz property.

In light of Proposition 5.1.5 and the remark thereafter, we can state the following result for commutative OBAs. Again, in view of Theorem 1.8.2 and Corollary 2.1.5, $r(a)$ in Proposition 5.3.5 is a Riesz point of $\sigma(a)$ relative to $\mathrm{N}(T)$, and therefore $p(a, r(a))$ exists. 
Proposition 5.3.5. Let $(A, C)$ be a commutative $O B A$ with closed algebra cone $C$ such that the spectral radius function in $(A, C)$ is monotone. Suppose that $T$ : $A \rightarrow B$ is a Banach algebra homomorphism with closed range satisfying the Riesz property and $a \in C$ is such that $r(a) \notin \sigma(T a)$. If $p(a, r(a)) \in \operatorname{span}(C \cap \mathrm{N}(T))$, then $r(a) \notin \beta_{T}^{+}(a)$.

The main reason for assuming that the OBA in Proposition 5.3.5 is commutative, is because of the identity $\omega_{T}^{+}(a)=\beta_{T}^{+}(a)$. It is not clear whether the assertion $r(a) \notin \beta_{T}^{+}(a)$ is true if we do away with the commutativity assumption; that is, we do not know whether Theorem 5.3.3 is valid under the weaker assumption of $p(a, r(a)) \in \operatorname{span}(C \cap \mathrm{N}(T))$.

We conclude this section with a discussion on semisimple OBAs. Let us recall that all positive elements of a finite-dimensional semisimple OBA $(A, C)$ have the upper Browder spectrum property relative to arbitrary Banach algebra homomorphisms acting on $A$ (see Corollary 5.2.11). Also, according to Theorem 5.3.3(iii), under certain natural conditions all positive elements of semisimple OBAs have the upper Browder spectrum property. It is important to note that, due to the assumption about the inverseclosedness of the algebra cone, this result does not suggest that all positive operators in $\mathcal{L}(E)$ have the upper Browder spectrum property.

It is natural to question the essentiality of the commutativity assumption next to the semisimple condition in Theorem 5.3.3(ii). (Of course our suspicion would dissapear if a positive operator on some Banach lattice which does not have the upper Browder spectrum property could be found.) Under this reduction, we note that a weaker conclusion is generally achieved: we are only able to get $r(a) \notin \beta_{T}^{+}(a u)$ for some positive element $u$ which is not necessarily the identity element.

Theorem 5.3.6. Let $(A, C)$ be a semisimple $O B A$ with closed algebra cone $C$ such that the spectral radius function in $(A, C)$ is monotone. Suppose that $T: A \rightarrow B$ is a Banach algebra homomorphism with closed range satisfying the Riesz property and $a \in C$. If $r(a) \notin \sigma(T a)$, then there exists $u \in C$ satisfying $a u=u a \neq 0$ such that $r(a) \notin \beta_{T}^{+}(a u)$.

Proof. Suppose that $r(a) \notin \sigma(\mathrm{Ta})$. In view of Theorem 1.8.2 and Corollary 2.1.5, let $r(a)$ be a pole of order, say, $k$ of $(\lambda \mathbf{1}-a)^{-1}$. From Theorem 1.8.1 and Lemma 2.2.7 we have that $a a_{-k} \in C \cap \mathrm{N}(T)$, so that $a a_{-k}-r(a) \mathbf{1} \in \mathcal{B}_{T}^{+}$and 
hence $r(a) \notin \beta_{T}^{+}\left(a a_{-k}\right)$, by Proposition 4.1.3. It remains to recall Theorem 1.8.1 again to finish this proof.

Whether $\beta_{T}^{+}(a u)$ in Theorem 5.3.6 can generally be replaced by $\beta_{T}^{+}(a)$ remains an open problem.

\subsection{Homomorphisms having the strong Riesz property}

The purpose for writing this section is to show that our results proven for homomorphisms with closed range satisfying the Riesz property have a stronger version. The main result here is Theorem 5.4.5.

We start our discussion by recalling the following definition introduced by Harte.

Definition 5.4.1 (Strong Riesz property of a homomorphism). ([20], (3.3)) $A$ Banach algebra homomorphism $T: A \rightarrow B$ is said to have the strong Riesz property if $\partial \sigma(a) \subseteq \sigma(T a) \cup$ iso $\sigma(a)$ for all $a \in A$.

Consequently, every Banach algebra homomorphism having the strong Riesz property satisfies the Riesz property. The following result is due to Mouton, Mouton and Raubenheimer.

Theorem 5.4.2. ([28], Corollary 7.9) If $T: A \rightarrow B$ is a Banach algebra homomorphism with closed range satisfying the Riesz property, then $T$ has the strong Riesz property.

In order to show that the assumption " $T$ has closed range and satisfies the Riesz property" in the results given in Section 5.3 can be relaxed to " $T$ has the strong Riesz property", we restate Corollary 2.1.5 in terms of homomorphisms with the latter property.

Lemma 5.4.3. Let $T: A \rightarrow B$ be a Banach algebra homomorphism satisfying the strong Riesz property and $a \in A$. Then the following statements are equivalent:

(i) $r(a) \in \sigma(a) \backslash \sigma(\mathrm{Ta})$.

(ii) $r(a) \in \sigma(a) \backslash \sigma(a+\overline{\mathrm{N}(T)})$.

(iii) $r(a)$ is a Riesz point of $\sigma(a)$ relative to $\mathrm{N}(T)$.

Moreover, if $A$ is a semisimple Banach algebra, then $r(a) \in \sigma(a) \backslash \sigma(T a)$ if and only if $r(a)>0$ is a pole of $(\lambda \mathbf{1}-a)^{-1}$ and $p(a, r(a)) \in \mathrm{N}(T)$. 
Proof. Since $T$ has the strong Riesz property, it has the Riesz property (i.e. $\mathrm{N}(T)$ is an inessential ideal of $A$ ), and therefore $\pi: A \rightarrow A / \overline{\mathrm{N}(T)}$ satisfies the Riesz property. Also, $\pi$ has closed range, and hence the identities

$$
\eta D(a, \mathrm{~N}(T))=\eta \sigma(\pi a)=\eta \beta_{\pi}(a)=\eta \beta_{T}(a)=\eta \sigma(T a)
$$

hold by Theorems 1.4.6, 1.5.7 and 1.5.8 and in view of ([37], Corollary 8.1).

Consequently, the equivalences of (i) through (iii) follow from (5.4.4). In addition, if $A$ is a semisimple Banach algebra, then the last assertion is clear from Lemma 1.4.5.

One of the most important consequences of Lemma 5.4.3 is that all results from this point onwards, stated for Banach algebra homomorphisms with closed range satisfying the Riesz property, remain true for Banach algebra homomorphisms having the strong Riesz property.

Next we state a stronger version of Theorems 5.3.3 and 5.3.6 and Proposition 5.3.5. Note that, in view of Theorem 1.8.2 and Lemma 5.4.3, we have, in the next result, that $r(a)$ is a Riesz point of $\sigma(a)$ relative to $\mathrm{N}(T)$, and therefore $p(a, r(a))$ exists.

Theorem 5.4.5. Let $(A, C)$ be an $O B A$ with closed algebra cone $C$ such that the spectral radius function in $(A, C)$ is monotone. Suppose that $T: A \rightarrow B$ is a Banach algebra homomorphism satisfying the strong Riesz property and $a \in C$ is such that $r(a) \notin \sigma(T a)$. Each of the following conditions gives $r(a) \notin \beta_{T}^{+}(a)$ :

(i) $p(a, r(a)) \in C$; hence any of the assertions (i) - (iii) in Theorem 5.3.3.

(ii) $A$ is commutative and $p(a, r(a)) \in \operatorname{span}(C \cap \mathrm{N}(T))$.

Furthermore, if $A$ is semisimple, then there exists $u \in C$ satisfying $a u=u a \neq 0$ such that $r(a) \notin \beta_{T}^{+}(a u)$.

Proof. (i) If any of the mentioned statements holds, then from Theorem 5.3.1 together with Corollary 5.3.2 we have the existence of $c \in C \cap \mathrm{N}(T) \cap$ $\operatorname{Comm}(a)$ such that $r(a) \notin \sigma(a+c)$. Hence $r(a) \notin \beta_{T}^{+}(a)$ in view of (4.1.5).

(ii) Suppose that $A$ is commutative and that $p(a, r(a)) \in \operatorname{span}(C \cap \mathrm{N}(T))$. Since the identity $\eta \sigma(T a)=\eta \beta_{T}(a)$ holds in view of (5.4.4), we have that $r(a) \in \sigma(a) \backslash \beta_{T}(a)$ by assumption. Following the second paragraph in the proof of Theorem 4.3.2, with $\lambda=r(a)$, gives $r(a) \notin \omega_{T}^{+}(a)=\beta_{T}^{+}(a)$.

The proof of the last statement uses the same argument as in the proof of Theorem 5.3.6; just replace Corollary 2.1.5 with Lemma 5.4.3. 
Some remarks: One of the reasons why we did not consider Banach algebra homomorphisms having the strong Riesz property from the beginning is because the notion of "strong Riesz" is less often used in the literature than the notion of "Riesz". We therefore continue to state our results (related to the upper Browder spectrum property) in terms of Banach algebra homomorphisms (with closed range) satisfying the Riesz property, though these results (as we will point out in the sequel) are valid for Banach algebra homomorphisms having the strong Riesz property.

\subsection{OBAs with disjunctive products}

Recall that $E$ denotes a Banach lattice and $\pi: \mathcal{L}(E) \rightarrow \mathcal{L}(E) / \mathcal{K}(E)$ the canonical homomorphism on $\mathcal{L}(E)$. Since $\omega_{\pi}(T)=\omega_{\pi}^{+}(T)$ for all $T \in \mathcal{L}(E)$, we have that $r(T) \notin \sigma_{e}(T)$ if and only if $r(T) \notin \omega_{\pi}^{+}(T)$. In ([4], Theorem 18) Alekhno gave concrete examples illustrating the implication $r(T) \notin$ $\sigma_{e}(T) \Rightarrow r(T) \notin \omega_{\pi}^{+}(T)$. In particular, we recall the following result.

Theorem 5.5.1. ([3], Theorem 4(c); [4], Theorem 18) Let E be either an AL-or a Dedekind complete AM-space with unit and $T$ be a positive operator on E. If $r(T) \notin \sigma_{e}(T)$ and $r(T)$ is a pole of order two of $(\lambda I-T)^{-1}$, then $r(T) \notin \sigma(T+$ $\left.\left(\alpha T_{-1}+n T_{-2}\right)^{+}\right)$for all $\alpha \in \mathbb{R}^{+} \backslash\{0\}$ and sufficiently large $n$ (depending on $\alpha$ ).

For $0 \leq S:=\left(\alpha T_{-1}+n T_{-2}\right)^{+} \in \mathcal{K}(E)$, since the identity $T S=S T$ may generally not hold, we cannot conclude from Theorem 5.5.1 that $r(T) \notin$ $\beta_{\pi}^{+}(T)$. It is still natural to ask whether the positive operators on Banach lattices as specified in Theorem 5.5.1 have the upper Browder spectrum property relative to $\pi$.

First note that, under the assumptions on the Banach lattice $E$ as stipulated in Theorem 5.5.1, $\mathcal{L}(E)\left(=\mathcal{L}^{r}(E)\right)$ becomes a Dedekind complete semisimple OBA with a disjunctive product. The aim of this section is to give sufficient conditions for positive elements of arbitrary Dedekind complete semisimple OBAs with disjunctive products to have the upper Browder spectrum property (relative to some prescribed Banach algebra homomorphism). As we shall see, an application of Theorem 5.5.4 (the principal result in this section) to the theory of regular operators provides a partial answer to the above-mentioned question (see Corollary 5.5.9). 
Combining Proposition 1.9.22 and Theorem 5.1.8, we can already state the following result for arbitrary Dedekind complete semisimple OBAs with disjunctive products.

Proposition 5.5.2. Let $(A, C)$ be a Dedekind complete semisimple OBA which has a disjunctive product with closed and proper algebra cone $C$ such that the spectral radius function in $(A, C)$ is monotone. Also, suppose that $T: A \rightarrow B$ is a Banach algebra homomorphism satisfying the strong Riesz property and is such that the spectral radius function in $(A / \overline{\mathrm{N}}(T), \pi C)$ is weakly monotone. If $a \in C$ is a spectrally order continuous element such that $r(a) \notin \sigma(T a)$ and $\left|a_{-1}\right|$ exists, then $r(a) \notin \sigma\left(a+\alpha\left|a_{-1}\right|\right)$ for all $0 \neq \alpha \in \mathbb{C}$.

Proof. Suppose that $a \in C$ is a spectrally order continuous element such that $r(a) \notin \sigma(T a)$ and $\left|a_{-1}\right|$ exists. From Theorem 1.8 .2 we have that $r(a) \in \sigma(a)$, and hence $r(a)$ is a Riesz point of $\sigma(a)$ relative to $\overline{\mathrm{N}(T)}$ in view of Lemma 5.4.3. The result then follows from Proposition 1.9.22 and Theorem 5.1.8.

Again, we point out that, in the case of operators on Banach lattices, $\left|T_{-1}\right|$ automatically exists and is an element of $\mathrm{N}(\pi)$ (see the remark below Theorem 5.1.7). This is, however, not true in the general case. Indeed, one of the disadvantages of the above result is that it requires the element $\left|a_{-1}\right|$ to exist; which is generally not the case. Moreover, even if $\left|a_{-1}\right|$ exists, note that $a_{-1}=p(a, r(a)) \in \mathrm{N}(T)$ (see Lemma 2.2.7) does not imply that $\left|a_{-1}\right| \in$ $\mathrm{N}(T)$, so that Proposition 5.5.2 does not propose that $r(a) \notin \omega_{T}^{+}(a)$ (and, therefore, neither that $r(a) \notin \beta_{T}^{+}(a)$ ). Thus, it would be interesting to obtain additional results where the existence of a modulus is not required. In the rest of the section we demonstrate how Alekhno's work (summarized in Section 1.9) enables us to make contributions in this direction.

Let us start by proving the following fact that was noted from the proof of Theorem 5.1.8 together with Lemma 1.9.27. We mention that, under the given assumptions, the Frobenius normal form in the next result exists by Theorem 1.9.26.

Theorem 5.5.3. Let $(A, C)$ be a Dedekind complete OBA which has a disjunctive product with closed and proper algebra cone. Suppose that $a \in C$ is a spectrally order continuous element such that $r(a)>0$ and $r(a)$ is an $f$-pole of $(\lambda \mathbf{1}-a)^{-1}$. If $\mathscr{C}:=\left\{\mathbf{1}=p_{n}, p_{n-1}, \ldots, p_{1}, p_{0}=0\right\}$ determines the Frobenius normal form of $a$ and $i \in\{1, \ldots, n\}$ is such that $r(a)=r\left(a_{q_{i}}\right)$, where $q_{i}:=p_{i} p_{i-1}^{d}$, then $r(a)$ is a simple pole of $\left(\lambda \mathbf{1}-a_{q_{i}}\right)^{-1}$. 
Proof. Let $i \in\{1, \ldots, n\}$ be such that $r\left(a_{q_{i}}\right)=r(a)$. We may assume that $p_{i} \neq p_{j}$ for all $i \neq j$. Then $0<q_{i} \in \mathbf{O I}(A)$ using Lemma 1.9.7. Since $0<a_{q_{i}} \leq a$ and $a \in A_{n}$, it follows from the remark following Definition 1.9.13 that $a_{q_{i}} \in A_{n}$. Also, since $r(a)$ is an $f$-pole of $(\lambda \mathbf{1}-a)^{-1}$, we have that $r\left(a_{q_{i}}\right)$ is a pole, say of order $m$, of $\left(\lambda \mathbf{1}-a_{q_{i}}\right)^{-1}$. Obviously $a_{q_{i}}$ is a block (and hence a spectral block) of $a$. Using the facts that $a$ is a spectrally order continuous element and $r(a)$ is a pole of order $m$ of $\left(\lambda \mathbf{1}-a_{q_{i}}\right)^{-1}$, we have that $\left(a_{q_{i}}\right)_{-m} \in A_{n}$. Seeing that $r\left(a_{q_{i}}\right)=r(a)$ and $\mathscr{C}$ determines the Frobenius normal form of $a$, the element $a_{q_{i}}$ is irreducible w.r.t. $q_{i}$. It is only left to note that $\mathbf{O I}(A)$ is Dedekind complete by Corollary 1.9.8; hence $r(a)$ is a simple pole of $\left(\lambda \mathbf{1}-a_{q_{i}}\right)^{-1}$ by Lemma 1.9.27.

We mention again that in the next result (and in Corollary 5.5.5) the Frobenius normal form of $a$ exists according to Corollary 2.1.6. Suppose that, in these results, the Frobenius normal form of $a$ is determined by $\mathscr{C}:=$ $\left\{\mathbf{1}=p_{n}, p_{n-1}, \ldots, p_{1}, p_{0}=0\right\}$ and let $q_{i}:=p_{i} p_{i-1}^{d}$.

It is worth noting that the following theorem presents a partial answer to the question of whether positive elements of Dedekind complete semisimple OBAs with disjunctive products have the upper Browder spectrum property.

Theorem 5.5.4. Let $(A, C)$ be a Dedekind complete semisimple OBA which has a disjunctive product with closed and proper algebra cone $C$ such that the spectral radius function in $(A, C)$ is monotone. Also, suppose that $T: A \rightarrow B$ is a Banach algebra homomorphism with closed range satisfying the Riesz property and is such that the spectral radius function in $(A / \overline{\mathrm{N}}(T), \pi C)$ is weakly monotone. If $a \in C$ is a spectrally order continuous element such that $r(a) \notin \sigma(T a)$, then $r(a) \notin$ $\cup_{i=1}^{n} \beta_{T}^{+}\left(a_{q_{i}}\right)$.

Proof. Suppose that $a \in C$ is a spectrally order continuous element such that $r(a) \notin \sigma(T a)$. Then $r(a)>0$ and $r(a)$ is a Riesz point of $\sigma(a)$ relative to $\mathrm{N}(T)$ by Theorem 1.8.2 and Corollary 2.1.5. By Proposition 1.9.22 $r(a)$ is an $f$-pole of $(\lambda \mathbf{1}-a)^{-1}$.

Since $0 \leq a_{q_{i}} \leq a$ and the spectral radius function in $(A, C)$ is monotone, we have that $r\left(a_{q_{i}}\right) \leq r(a)$. If $r\left(a_{q_{i}}\right)<r(a)$, then $r(a) \notin \sigma\left(a_{q_{i}}\right)$, and hence $r(a) \notin \beta_{T}^{+}\left(a_{q_{i}}\right)$ by Proposition 4.1.11.

If $r\left(a_{q_{i}}\right)=r(a)$, then from Theorem 5.5.3 it follows that $r\left(a_{q_{i}}\right)$ is a simple pole of $\left(\lambda \mathbf{1}-a_{q_{i}}\right)^{-1}$. By using the fact that the spectral radius function in 
$(A / \overline{\mathrm{N}}(T), \pi C)$ is weakly monotone, together with Theorem 1.8.3 and Corollary 2.1.4, it follows from $0 \leq a_{q_{i}} \leq a$ that

$$
r\left(T a_{q_{i}}\right)=r\left(\pi a_{q_{i}}\right) \leq r(\pi a)<r(a)=r\left(a_{q_{i}}\right) .
$$

Hence $r\left(a_{q_{i}}\right) \notin \sigma\left(T a_{q_{i}}\right)$. From Theorem 5.3.3(i) it follows that $r(a)=r\left(a_{q_{i}}\right) \notin$ $\beta_{T}^{+}\left(a_{q_{i}}\right)$.

We have thus shown that $r(a) \notin \beta_{T}^{+}\left(a_{q_{i}}\right)$ for all $i \in\{1, \ldots, n\}$. This completes our proof.

Some remarks: Note that the element $a_{q_{i}}$ equals $p_{i-1}^{d} a p_{i}$, and therefore $r(a) \notin \cup_{i=1}^{n} \beta_{T}^{+}\left(p_{i-1}^{d} a p_{i}\right)$ by Theorem 5.5.4. Hence, in the case where, for instance, $i=1$, we have that $r(a) \notin \beta_{T}^{+}\left(a p_{1}\right)$, and for the case $i=n$, it follows that $r(a) \notin \beta_{T}^{+}\left(p_{n-1}^{d} a\right)$.

Our next result is a special case of Theorem 5.5.4.

Corollary 5.5.5. Let $(A, C)$ be a Dedekind complete semisimple OBA which has a disjunctive product with closed and proper algebra cone $C$ such that the spectral radius function in $(A, C)$ is monotone. Also, suppose that $T: A \rightarrow B$ is a Banach algebra homomorphism with closed range satisfying the Riesz property and is such that the spectral radius function in $(A / \overline{\mathrm{N}(T)}, \pi C)$ is weakly monotone. Let $a \in C$ be a spectrally order continuous element such that $r(a) \notin \sigma(T a)$. If $a \in q_{i} A q_{i}$ for some $i \in\{1, \ldots, n\}$, then $r(a) \notin \beta_{T}^{+}(a)$.

Proof. Let $i \in\{1, \ldots, n\}$ be such that $a \in q_{i} A q_{i}$. Then $a=a_{q_{i}}$, and hence from Theorem 5.5.4 it follows that $r(a) \notin \beta_{T}^{+}(a)$.

We mention that, under the assumptions as specified in Corollary 5.5.5, the equality $\beta_{T}^{+}(a)=\cup_{i=1}^{n} \beta_{T}^{+}\left(a_{q_{i}}\right)$ holds. Also, since $a=a_{q_{i}}$ is true for some $i$ (and therefore $r(a)=r\left(a_{q_{i}}\right)$ ), we have from Theorem 5.5.3 (see also Proposition 1.9.22) that $r(a)$ is a simple pole of $(\lambda \mathbf{1}-a)^{-1}$; in which case the condition $r(a) \notin \beta_{T}^{+}(a)$ follows, alternatively, from Theorem 5.3.3(i).

Remark 5.5.6. We mention that in the special case where $\mathscr{C}:=\left\{\mathbf{1}=p_{1}, 0=p_{0}\right\}$ determines the Frobenius normal form of $a$, we have that $a \in A=q_{1} A q_{1}$, so that the implication $r(a) \notin \sigma(\mathrm{Ta}) \Rightarrow r(a) \notin \beta_{T}^{+}(a)$ holds by Corollary 5.5.5.

As yet we do not know if alternative (more general) conditions exist under which $\cup_{i=1}^{n} \beta_{T}^{+}\left(a_{q_{i}}\right)$ in Theorem 5.5.4 can be replaced by $\beta_{T}^{+}(a)$. 
Remark 5.5.7. By replacing Corollaries 2.1.4 and 2.1.5 and Theorem 5.3.3(i) in the proof of Theorem 5.5.4 by (5.4.4), Lemma 5.4.3 and Theorem 5.4.5(i), respectively, Theorem 5.5.4 (and, therefore, Corollary 5.5.5) can be extended to Banach algebra homomorphisms having the strong Riesz property.

If $E$ is a Dedekind complete Banach lattice, then $\mathcal{L}^{r}(E)$ is a well-known example of a Dedekind complete semisimple OBA with a disjunctive product. We end this section by giving several applications of Theorem 5.5.4 to the theory of regular operators.

In view of the remark preceding Theorem 5.5.4, together with Remark 1.9.17 and the statement preceding it, the Frobenius normal form of $T \in$ $\mathcal{L}^{r}(E)$ in Corollary 5.5.8 exists. By recalling Remark 1.9.25 and the observation thereafter, let $\left\{E=B_{n}, B_{n-1}, \ldots, B_{0}=\{0\}\right\}$ be the $T$-invariant chain of projection bands in the Frobenius normal form of the positive operator $T$ in Corollaries 5.5.8 and 5.5.9 and let $Q_{i}=B_{i} \cap B_{i-1}^{d}$.

Keep in mind the notation $\pi_{r}: \mathcal{L}^{r}(E) \rightarrow \mathcal{L}^{r}(E) / \mathcal{K}^{r}(E)$ and the identity $r\left(T, \mathcal{L}^{r}(E)\right)=r(T, \mathcal{L}(E))$ for all positive operators $T$ on $E$.

Corollary 5.5.8. Let $E$ be a Dedekind complete Banach lattice and $T$ be a positive spectrally order continuous operator on E. If $r(T) \notin \sigma_{o e}(T)$, then $r(T) \notin$ $\cup_{i=1}^{n} \beta_{\pi_{r}}^{+}\left(P_{Q_{i}} T P_{Q_{i}}, \mathcal{L}^{r}(E)\right)$.

Proof. Since $E$ is a Dedekind complete Banach lattice, we have that $\mathcal{L}^{r}(E)$ is a Dedekind complete OBA under the $r$-norm, by the remark following Example 1.9.4, and has a disjunctive product, by the paragraph following Definition 1.9.20. It is also known that, for $E$ Dedekind complete, the spectral radius function in $\left(\mathcal{L}^{r}(E) / \mathcal{K}^{r}(E), \pi_{r} K\right)$, where $K$ is the cone of positive operators on $E$, is weakly monotone ([27], Theorem 2.8). If $T$ is a positive spectrally order continuous operator on $E$, then $T$ is a spectrally order continuous element in $\mathcal{L}^{r}(E)$ in view of Remark 1.9.17 and the fact preceding this remark. The desired result then follows from Theorem 5.5.4 and (1.9.24).

Making use of Corollary 5.5.8, we have the following:

Corollary 5.5.9. Let $E$ be a Dedekind complete Banach lattice and $T$ be a positive operator on $E$ such that $r(T) \notin \sigma_{e}(T)$. Under each of the following assumptions we have that $r(T) \notin \cup_{i=1}^{n} \beta_{\pi}^{+}\left(P_{Q_{i}} T P_{Q_{i}}, \mathcal{L}(E)\right)$ : 
(i) E is an AL-space.

(ii) $E$ is an $A M$-space with unit and $T$ is an order continuous operator on $E$.

Proof. If any of the statements (i) - (ii) on the Banach lattice $E$ hold, then $\mathcal{L}(E)=\mathcal{L}^{r}(E)$ (recall the remark following Example 1.9.4) and $\mathcal{K}(E)=$ $\mathcal{K}^{r}(E)$ (in view of Lemma 1.6.9), so that $\pi=\pi_{r}$.

(i) Suppose that $E$ is an $A L$-space. Since $E$ has order continuous norm (see (1.6.7)), we have that $(\mathcal{L}(E))_{n}=K$, where $K$ is the algebra cone of all positive operators on $E$. From Proposition 1.9.18 it follows that $T$ is a spectrally order continuous element in $\mathcal{L}(E)$, and hence a spectrally order continuous operator on $E$, from the paragraph just below Definition 1.9.16. The result then follows from Corollary 5.5.8.

(ii) Suppose now that $E$ is an $A M$-space with unit and that $T$ is an order continuous operator on $E$. Then $T$ is a spectrally order continuous operator on $E$ by Lemma 1.9.19, and hence the result follows from Corollary 5.5.8.

Remark 5.5.10. From Corollary 5.5.9 we have, for $i=1$ and $i=n$, that $r(T) \notin$ $\beta_{\pi}^{+}\left(T P_{B_{1}}, \mathcal{L}(E)\right)$ and $r(T) \notin \beta_{\pi}^{+}\left(P_{B_{n-1}} T, \mathcal{L}(E)\right)$, respectively.

Comparing Corollary 5.5.9 with Theorem 5.5.1, it is worth pointing out that this corollary presents a partial result to the question of whether the operators considered have the upper Browder spectrum property. 


\section{Chapter 6}

\section{The lower Weyl and Lozanovsky spectra}

The lower Weyl and Lozanovsky spectra were investigated in, for instance, [3], [4] and [5]. Originally, these spectra have been studied for positive bounded linear operators acting on Banach lattices.

The main purpose of this chapter is to introduce and investigate these spectra for arbitrary positive elements of general OBAs. In particular, in Section 6.1, we will address in an OBA-context the problem of developing conditions under which the spectral radius of a positive element will be outside of the lower Weyl spectrum of the element if it is known to be outside of the Fredholm spectrum of that element. In Section 6.2 we present some results on the Lozanovsky spectrum of a positive OBA element. We point out that the results presented in this chapter are the OBA versions of the results established by Alekhno in the operator context.

In the sequel of this chapter it is always assumed that $E$ is a Banach lattice and that $\pi: \mathcal{L}(E) \rightarrow \mathcal{L}(E) / \mathcal{K}(E)$ is the canonical homomorphism on $\mathcal{L}(E)$.

\subsection{Introducing the lower Weyl spectrum}

Recall that, for $0 \leq T \in \mathcal{L}(E)$, the set

$$
\omega_{\pi}^{-}(T):=\bigcap\{\sigma(T-K): 0 \leq K \leq T \text { and } K \in \mathcal{K}(E)\}
$$

is called the lower Weyl spectrum of $T$ ([3], p.376).

The set of inclusions $\sigma_{e}(T) \subseteq \omega_{\pi}^{-}(T) \subseteq \sigma(T)$ were established in ([3], p.376) and an example illustrating that these inclusions are generally strict 
were also given (see [4], Example 19). Consequently, a central problem being investigated by Alekhno is the following: given that the spectral radius of $T$ is outside its essential spectrum, what conditions suffice for it to be outside the lower Weyl spectrum of $T$ ? Some contributions to the solution of this problem can be found in ([3], Theorem 5) and ([4], Theorem 20 and Corollary 21).

Definition 6.1.1 (Lower Weyl). Let $(A, C)$ be an $O B A$ and $T: A \rightarrow B$ be a Banach algebra homomorphism. The lower Weyl spectrum of $a \in C$, denoted by $\omega_{T}^{-}(a)$, is defined as follows:

$$
\omega_{T}^{-}(a):=\bigcap_{\substack{0 \leq c \leq a \\ c \in \mathrm{N}(T)}} \sigma(a-c)
$$

The lower Weyl spectrum contains the Weyl spectrum, as can be seen in the following result.

Proposition 6.1.2. Let $(A, C)$ be an $O B A$ and $T: A \rightarrow B$ be a Banach algebra homomorphism. If $a \in C$, then

$$
\sigma(T a) \subseteq \omega_{T}(a) \subseteq \omega_{T}^{-}(a) \subseteq \sigma(a)
$$

and hence

$$
\sigma(T a) \subseteq \omega_{T}(a) \subseteq \omega_{T}^{+}(a) \cap \omega_{T}^{-}(a) \subseteq \omega_{T}^{+}(a) \cup \omega_{T}^{-}(a) \subseteq \sigma(a)
$$

Moreover, if $T$ has the Riesz property, then

$$
\sigma(T a) \subseteq \omega_{T}(a) \subseteq \omega_{T}^{+}(a) \subseteq \omega_{T}^{-}(a) \subseteq \sigma(a)
$$

Proof. Let $a \in C$ and suppose that $\lambda \notin \omega_{T}^{-}(a)$. Then there exists $c \in \mathrm{N}(T)$ satisfying $0 \leq c \leq a$ such that $\lambda \notin \sigma(a-c)$. Since $-c \in \mathrm{N}(T)$, we have from the representation of $\omega_{T}(a)$ below Proposition 1.5.5 that $\lambda \notin \omega_{T}(a)$. Hence $\omega_{T}(a) \subseteq \omega_{T}^{-}(a)$. The first inclusion is clear from (1.5.6), while the third inclusion follows from the definition of $\omega_{T}^{-}(a)$ when choosing $c=0$.

The second set of inclusions follows from Proposition 4.1.11 and the first part of the result.

Now, if $T$ has the Riesz property, then $\omega_{T}^{+}(a) \subseteq \omega_{T}^{-}(a)$ in view of Corollary 4.1.10. It remains to recall (6.1.3) to finish this proof. 
We remark that (6.1.3) was found by Alekhno in the setting of positive operators on Banach lattices (see [3], p.376). Although it appears from [3] that Alekhno was not aware of the inclusion $\omega_{\pi}^{+}(T) \subseteq \omega_{\pi}^{-}(T)$, he established (6.1.4) in [4] via Theorem 16.

The following properties of the lower Weyl spectrum follow easily.

Theorem 6.1.5. Let $(A, C)$ be an $O B A$ and $T: A \rightarrow B$ be a Banach algebra homomorphism. If $a \in C$, then $\omega_{T}^{-}(a)$ is non-empty and compact.

Proof. Using Proposition 6.1.2, this proof follows the arguments of the proof of Corollary 4.1.12.

The following examples illustrate that the inclusions in Proposition 6.1.2 (or, more specifically, (6.1.4)) can be strict. Note that from ([4], Example 19) we have a positive operator $T$ satisfying $\omega_{\pi}(T) \neq \omega_{\pi}^{-}(T)$. Our next example shows that this statement is generally true even for elements of a finite-dimensional OBA.

Example 6.1.6. Consider the homomorphism $T: M_{2}^{u}(\mathbb{C}) \rightarrow \mathbb{C}$ defined by

$$
T\left[\begin{array}{cc}
x_{11} & x_{12} \\
0 & x_{22}
\end{array}\right]=x_{11}
$$

and $X:=\left(\begin{array}{ll}2 & 0 \\ 0 & 0\end{array}\right) \in M_{2}^{u}(\mathbb{C})$. Then $\omega_{T}(X)=\omega_{T}^{+}(X) \subsetneq \omega_{T}^{-}(X)$.

The equality $\sigma(T X)=\{2\}$ is clear, and hence $\omega_{T}(X)=\omega_{T}^{+}(X)=\{2\}$ follows from Example 3.2.12. Furthermore, since $\left(\begin{array}{ll}0 & 0 \\ 0 & 0\end{array}\right)$ is the only element in $M_{2}^{u}\left(\mathbb{R}^{+}\right) \cap \mathrm{N}(T)$ dominated by $X$, we have that $\omega_{T}^{-}(X)=\sigma(X)=\{0,2\}$, and hence the result follows.

Example 6.1.7. Let $l^{\infty}:=l^{\infty}(\mathbb{C})$ and consider the homomorphism $T: l^{\infty} \rightarrow l^{\infty}$ defined by $T\left(x_{1}, x_{2}, \ldots\right)=\left(x_{2}, x_{3}, \ldots\right)$. For $a:=(1,0,0, \ldots) \in l^{\infty}$, we have that $\omega_{T}^{-}(a) \subsetneq \sigma(a)$.

The set of identities

$$
\omega_{T}^{-}(a)=\bigcap_{\substack{0 \leq c \leq a \\ c \in \mathrm{N}(T)}} \sigma(a-c)=\bigcap_{0 \leq x \leq 1} \sigma((1-x, 0,0, \ldots))=\bigcap_{0 \leq x \leq 1}\{1-x, 0\}=\{0\}
$$

follows easily, and hence $\sigma(T a)=\omega_{T}(a)=\omega_{T}^{-}(a)=\{0\} \subsetneq\{0,1\}=\sigma(a)$ by also recalling Proposition 6.1.2. 
An interesting aspect of research in OBAs is that of investigating in an OBAcontext certain problems that originated in $\mathcal{L}(E)$. Motivated by ([3], Section $3)$, an arbitrary positive element $a$ of a general OBA $(A, C)$ is said to have the lower Weyl spectrum property (relative to a Banach algebra homomorphism $T$ from $A$ to an arbitrary Banach algebra $B)$ if $r(a) \notin \sigma(T a)$ implies that $r(a) \notin \omega_{T}^{-}(a)$. In view of ([5], Example 4.6), not all positive OBA elements have the lower Weyl spectrum property.

Throughout we shall recall some examples (provided by Alekhno) of positive operators which have the lower Weyl spectrum property, with the aim of presenting generalizations and analogues of Alekhno's results. It is worth pointing out that the results given here are established with simpler proofs, although they are weaker.

For the purpose of our discussion that follows, suppose that $T$ is a positive operator on $E$ such that $r(T) \notin \sigma_{e}(T)$ and there exists a sequence $\left(K_{n}\right)$ of positive compact operators such that $K_{n} x \uparrow T x$ for all $x \in E_{+}$.

In ([3], Theorem 5(b)) Alekhno showed that the assertion $r(T) \notin \omega_{\pi}^{-}(T)$ is true whenever $E$ has order continuous norm. Now, for a Banach lattice $E$ with order continuous norm, it is an easy exercise to show that the statement $K_{n} x \uparrow T x$ for all $x \in E_{+}$is equivalent to $\left(T-K_{n}\right) x \downarrow 0$ for all $x \in E_{+}$ (in fact only the Dedekind completeness of $E$ is used). It then follows from a theorem of Nakano (see [7], Theorem 12.9) that the latter property implies that $\left\|\left(T-K_{n}\right) x\right\| \downarrow 0$ for all $x \in E_{+}$, and hence $\lim _{n \rightarrow \infty}\left\|\left(T-K_{n}\right) x\right\|=0$ for all $x \in E_{+}$. We point out here, for the sake of interest, that even under the weaker assumption of " $\left(K_{n}\right)$ is an increasing sequence satisfying $\lim _{n \rightarrow \infty}\left\|\left(T-K_{n}\right) x\right\|=0$ for all $x \in E_{+}$" Alekhno showed in ([4], Corollary 21) that the statement $r(T) \notin \omega_{\pi}^{-}(T)$ is true.

The next result can be viewed as an OBA version of ([3], Theorem 5(b)).

Proposition 6.1.8. Let $(A, C)$ be an $O B A$ and $T: A \rightarrow B$ be a Banach algebra homomorphism. Suppose that $a \in C$ is such that there exists a sequence $\left(c_{n}\right)$ in $C \cap \mathrm{N}(T)$ satisfying $c_{n} \leq$ a for all $n \in \mathbb{N}$ and $\lim _{n \rightarrow \infty}\left\|c_{n}-a\right\|=0$. If $r(a) \notin \sigma(T a)$, then $r(a) \notin \omega_{T}^{-}(a)$.

Proof. Suppose that $r(a) \notin \sigma(T a)$. If $T$ is bounded, then $0 \neq a \in \mathrm{N}(T)$, and hence

$$
\omega_{T}^{-}(a)=\bigcap_{\substack{0 \leq c \leq a \\ c \in \mathrm{N}(T)}} \sigma(a-c) \subseteq \sigma(a-a)=\{0\}=\sigma(T a),
$$


in which case the result obviously holds.

The proof for the general case is by contradiction. Suppose that $r(a) \in$ $\omega_{T}^{-}(a)$. Then $r(a) \in \sigma\left(a-c_{n}\right)$ for all $n \in \mathbb{N}$, and hence $r(a) \leq r\left(a-c_{n}\right) \leq$ $\left\|a-c_{n}\right\|$ for all $n \in \mathbb{N}$, so that $0<r(a) \leq \lim _{n \rightarrow \infty}\left\|a-c_{n}\right\|=0$. This is impossible; therefore $r(a) \notin \omega_{T}^{-}(a)$.

We point out that in the above proof we could have assumed, in fact, that $c_{n} \leq a$ only for sufficiently large $n$.

In some cases (as can be seen in the proof of Proposition 6.1.8 for $T$ bounded) the condition $\lim _{n \rightarrow \infty} c_{n}=a$ implies that $a \in \mathrm{N}(T)$, in which case $\omega_{T}^{-}(a)=\{0\}$.

It is interesting to note that Proposition 6.1.8 provides yet additional sufficient conditions for a positive element in a commutative OBA to have the upper Browder spectrum property. We have the following:

Corollary 6.1.9. Let $(A, C)$ be a commutative $O B A$ and $T: A \rightarrow B$ be a Banach algebra homomorphism which satisfies the Riesz property. Suppose that $a \in C$ is such that there exists a sequence $\left(c_{n}\right)$ in $C \cap \mathrm{N}(T)$ satisfying $c_{n} \leq$ a for all $n \in \mathbb{N}$ and $\lim _{n \rightarrow \infty}\left\|c_{n}-a\right\|=0$. If $r(a) \notin \sigma($ Ta $)$, then $r(a) \notin \beta_{T}^{+}(a)$.

Proof. The result follows from Proposition 6.1.8, equation (6.1.4) and the fact that $\omega_{T}^{+}(a)=\beta_{T}^{+}(a)$.

We point out that, if $r(T)$ is a simple pole of $(\lambda I-T)^{-1}$, then from ([3], Theorem 5(a)) we have that $r(T) \notin \omega_{\pi}^{-}(T)$. Unfortunately, at this stage, we have not managed to come up with an OBA version of ([3], Theorem 5(a)).

We conclude this section with an OBA version of ([4], Theorem 20(b)). But first we discuss the aforementioned statement in more detail: one of the assumptions in ([4], Theorem 20(b)) is that the order continuous dual $E_{n}^{\sim}$ separates the points of the Banach lattice $E$. This condition, however, does not give us information on the OBA $\mathcal{L}(E)$ itself. In view of (1.6.7), ([7], Theorem 12.14) and ([35], Proposition 8.3(ii), p.113), we observe that the condition " $E_{n}^{\sim}$ separates the points of $E$ " holds whenever $E$ is an $A L$ space. As is well-known, the latter statement implies that $\mathcal{L}(E)=\mathcal{L}^{r}(E)$ is a Dedekind complete semisimple OBA with a disjunctive product (recall the remarks following Example 1.9.4 and Remark 5.5.7). Consequently, our next result can be viewed as a generalization of ([4], Theorem 20(b)) in the case where $E$ is an $A L$-space. We point out that this result is a consequence of ([6], Corollary 5.4). 
Theorem 6.1.10. Let $(A, C)$ be a Dedekind complete semisimple OBA which has a disjunctive product with closed and normal algebra cone $C$. Also, suppose that $T: A \rightarrow B$ is a Banach algebra homomorphism with closed range satisfying the Riesz property and is such that the spectral radius function in $(A / \overline{\mathrm{N}(T)}, \pi C)$ is weakly monotone. If $a \in C$ is a spectrally order continuous element such that there exists a net $\left(c_{\alpha}\right)$ in $C \cap \mathrm{N}(T)$ satisfying $c_{\alpha} \uparrow a$, then $r(a) \notin \sigma(T a)$ implies that $r(a) \notin \omega_{T}^{-}(a)$.

Proof. Under the specified conditions, we have that $r(a)>0$ and $r(a)$ is a Riesz point of $\sigma(a)$ relative to $\mathrm{N}(T)$ by Theorem 1.8.2 and Corollary 2.1.5. In view of Proposition 1.9.22 and ([6], Corollary 5.4), we can find $\alpha_{0}$ such that $r\left(a-c_{\alpha_{0}}\right)<r(a)$ for all $\alpha \geq \alpha_{0}$. Hence $r(a) \notin \sigma\left(a-c_{\alpha_{0}}\right)$ for all $\alpha \geq \alpha_{0}$, so that $r(a) \notin \omega_{T}^{-}(a)$.

By replacing Corollary 2.1.5 in the proof of Theorem 6.1.10 with Lemma 5.4.3, this result remains true even under the weaker assumption of " $T$ has the strong Riesz property". We also remark that the condition " $C$ is normal" in Theorem 6.1 .10 can be replaced by the weaker condition " $\mathrm{C}$ is proper and the spectral radius function in $(A, C)$ is monotone".

Next, we apply Theorem 6.1.10 to $\mathcal{L}^{r}(E)$. The reader is reminded about the notation $\pi_{r}: \mathcal{L}^{r}(E) \rightarrow \mathcal{L}^{r}(E) / \mathcal{K}^{r}(E)$ and the identity $r\left(T, \mathcal{L}^{r}(E)\right)=$ $r(T, \mathcal{L}(E))$ for all $0 \leq T \in \mathcal{L}(E)$.

Corollary 6.1.11. Let $E$ be a Dedekind complete Banach lattice and $T \geq 0$ be a spectrally order continuous operator on E such that there exists a net $\left(K_{\alpha}\right)$ of positive r-compact operators satisfying $K_{\alpha} x \uparrow$ Tx for all $x \in E_{+}$. If $r(T) \notin \sigma_{o e}(T)$, then $r(T) \notin \omega_{\pi_{r}}^{-}(T)$.

Proof. A glance at the proof of Corollary 5.5.8 reveals that all conditions in Theorem 6.1.10 are satisfied. Also, for a Dedekind complete Banach lattice, it is an easy exercise to show that the statement " $K_{\alpha} x \uparrow T x$ for all $x \in E_{+}$" is equivalent to the condition " $K_{\alpha} \uparrow T$ in $\mathcal{L}^{r}(E)$ ". It remains to recall Theorem 6.1.10 to finish this proof.

Corollary 6.1.11 applied to $\mathcal{L}(E)$, for a Dedekind complete AM-space $E$ with unit, yields the following:

Corollary 6.1.12. Let $E$ be a Dedekind complete AM-space with unit and $T$ be a positive order continuous operator on $E$ such that there exists a net $\left(K_{\alpha}\right)$ of positive 
compact operators satisfying $K_{\alpha} x \uparrow T x$ for all $x \in E_{+}$. If $r(T) \notin \sigma_{e}(T)$, then $r(T) \notin \omega_{\pi}^{-}(T)$.

Proof. The result follows from Corollary 6.1.11 and a glance at the proof of Corollary 5.5.9(ii).

Example 4.6 in [5] shows that in general Corolary 6.1 .12 cannot be extended to arbitrary positive operators on $E$.

Some remarks: Of course, in view of (1.6.7) and ([3], Theorem 5(b)), the previous result is true if we consider an $A L$-space $E$ and an arbitrary positive operator $T$ on $E$. However, it is not clear whether Corollary 6.1.12 follows via duality, as we do not know if the lower Weyl spectrum of $T$ and its adjoint $T^{*}$ (under the assumptions in Corollary 6.1.12) coincide (the reader might want to look at ([5], Theorem 2.1)). We point out that, in general, the lower Weyl spectrum of $T^{*}$ is contained in the lower Weyl spectrum of $T$ ([5], p.305).

\subsection{A note on the Lozanovsky spectrum}

Motivated by a theorem of Lozanovsky ([2], p.199), Alekhno ([3], p.384) introduced the Lozanovsky essential spectrum of a positive operator $T$ on a Banach lattice $E$ as the set

$$
\sigma_{e l}(T):=\bigcap_{\substack{0 \leq Q \leq T \\ Q \leq K \text { for some } K \in \mathcal{K}(E)}} \sigma(T-Q) .
$$

Definition 6.2.1 (Lozanovsky spectrum). Let $(A, C)$ be an $O B A$ and $T: A \rightarrow$ $B$ be a Banach algebra homomorphism. The Lozanovsky spectrum of $a \in C$, denoted by $\omega_{T}^{L}(a)$, is defined as follows:

$$
\omega_{T}^{L}(a):=\bigcap_{\substack{0 \leq c \leq a \\ c \leq b \text { for some } b \in \mathrm{N}(T)}} \sigma(a-c)
$$

By definition, the Lozanovsky spectrum is a compact subset of the complex plane. However, it is not evident whether the Lozanovsky spectrum is a non-empty set in general. Interestingly enough, even in the case of positive operators on Banach lattices, this is still an open problem (see [4], p.18). In Remark 6.2.9 we point out conditions under which the Lozanovsky spectrum is a non-empty set. 
Obviously, the Lozanovsky spectrum is contained in the lower Weyl spectrum, but the relationship between the Weyl and Lozanovsky spectra is not immediately clear. We proceed to investigate this relationship in an OBA-context. Firstly, let us examine the implication

$$
r(a) \in \omega_{T}(a)(\operatorname{or} r(a) \in \sigma(T a)) \Rightarrow r(a) \in \omega_{T}^{L}(a)
$$

as was done in the operator setting. We start by pointing out the following auxiliary result.

Lemma 6.2.2. Let $(A, C)$ be an $O B A$ with closed algebra cone $C$ such that the spectral radius function in $(A, C)$ is monotone. Also, suppose that $T: A \rightarrow$ $B$ is a Banach algebra homomorphism such that the spectral radius function in $(A / \overline{\mathrm{N}}(T), \pi C)$ is weakly monotone. If $a \in C$ is such that $r(a) \notin \omega_{T}^{L}(a)$, then $r(a) \mathbf{1}-a+\overline{\mathrm{N}(T)} \in(A / \overline{\mathrm{N}(T)})^{-1}$.

Proof. Let $a \in C$ be such that $r(a) \notin \omega_{T}^{L}(a)$. Then there exists $c \in C$ such that $c \leq b$ for some $b \in \mathrm{N}(T), c \leq a$ and $r(a) \notin \sigma(a-c)$, that is, $d:=r(a) \mathbf{1}-$ $(a-c) \in A^{-1}$. Since $0 \leq a-c \leq a$ and the spectral radius function in $(A, C)$ is monotone, the inequality $r(a-c) \leq r(a)$ holds. For $a-c \in C$, we have from Theorem 1.8.2 that $r(a-c) \in \sigma(a-c)$, and hence $r(a-c)<r(a)$ as $r(a) \notin \sigma(a-c)$. From Proposition 1.8.6 it follows that $d^{-1}=[r(a) \mathbf{1}-(a-$ $c)]^{-1} \in C$, and hence $0 \leq d^{-1} c \leq d^{-1} b$. Since $d^{-1} b \in \overline{\mathrm{N}(T)}$, the element $d^{-1} b$ is Riesz w.r.t. $\overline{\mathrm{N}(T)}$, so that $d^{-1} c$ is Riesz w.r.t. $\overline{\mathrm{N}(T)}$ by Theorem 1.8.4. By also using the spectral mapping theorem, we obtain that

$$
\sigma\left(\mathbf{1}-d^{-1} c+\overline{\mathrm{N}(T)}\right)=1+\sigma\left(-d^{-1} c+\overline{\mathrm{N}(T)}\right)=\{1\},
$$

and hence $\mathbf{1}-d^{-1} c+\overline{\mathrm{N}(T)} \in(A / \overline{\mathrm{N}(T)})^{-1}$. Finally,

$$
\begin{aligned}
r(a) \mathbf{1}-a+\overline{\mathrm{N}(T)} & =d-c+\overline{\mathrm{N}(T)} \\
& =d\left(\mathbf{1}-d^{-1} c\right)+\overline{\mathrm{N}(T)} \\
& =(d+\overline{\mathrm{N}(T)})\left(\mathbf{1}-d^{-1} c+\overline{\mathrm{N}(T)}\right) \in(A / \overline{\mathrm{N}(T)})^{-1}
\end{aligned}
$$

This completes the proof.

Since $\pi: \mathcal{L}(E) \rightarrow \mathcal{L}(E) / \mathcal{K}(E)$ is both bounded and a homomorphism with closed range satisfying the Riesz property, the following two results (Theorems 6.2.3 and 6.2.4) present OBA versions of ([3], Theorem 7). We 
remark that, due to the assumption about the weak monotonicity, these results are not generalizations in the strict sense.

Although Theorem 7 in [3] is formulated in terms of the Fredholm spectrum, this result remains true whenever the Fredholm spectrum is replaced by the (upper) Weyl spectrum. In view of Theorem 1.5.8, we formulate the following result (for Banach algebra homomorphisms with closed range satisfying the Riesz property) in terms of the Weyl spectrum.

Theorem 6.2.3. Let $(A, C)$ be an $O B A$ with closed algebra cone $C$ such that the spectral radius function in $(A, C)$ is monotone. Also, suppose that $T: A \rightarrow B$ is a Banach algebra homomorphism with closed range satisfying the Riesz property such that the spectral radius function in $(A / \overline{\mathrm{N}}(T), \pi C)$ is weakly monotone. If $a \in C$ is such that $r(a) \in \omega_{T}(a)$, then $r(a) \in \omega_{T}^{L}(a)$.

Proof. Let $a \in C$ and suppose that $r(a) \notin \omega_{T}^{L}(a)$. From Lemma 6.2.2 it follows that $r(a) \mathbf{1}-a+\overline{\mathrm{N}(T)} \in(A / \overline{\mathrm{N}(T)})^{-1}$, that is $r(a) \notin \sigma(a+\overline{\mathrm{N}(T)})$, so that $r(a) \notin \sigma(\mathrm{Ta})$ by Corollary 2.1.5. By recalling Theorem 1.5.8 the proof is complete.

By replacing Corollary 2.1.5 and Theorem 1.5.8, respectively, by Lemma 5.4.3 and the last identity of (5.4.4) (recall also (1.5.6)), Theorem 6.2.3 remains valid for Banach algebra homomorphisms having the strong Riesz property.

For a bounded homomorphism we have the following:

Theorem 6.2.4. Let $(A, C)$ be an $O B A$ with closed algebra cone $C$ such that the spectral radius function in $(A, C)$ is monotone. Also, suppose that $T: A \rightarrow B$ is a bounded Banach algebra homomorphism such that the spectral radius function in $(A / \mathrm{N}(T), \pi C)$ is weakly monotone. If $a \in C$ is such that $r(a) \in \sigma(T a)$, then $r(a) \in \omega_{T}^{L}(a)$.

Proof. Let $a \in C$ and suppose that $r(a) \notin \omega_{T}^{L}(a)$. By Lemma 6.2.2 we have that $r(a) \mathbf{1}-a+\mathrm{N}(T) \in(A / \mathrm{N}(T))^{-1}$, and hence $r(a) \mathbf{1}-T a=\phi(r(a) \mathbf{1}-a+$ $\mathrm{N}(T)) \in B^{-1}$, where $\phi: A / \mathrm{N}(T) \rightarrow \overline{T(A)} \subseteq B$ is defined by $\phi(a+\mathrm{N}(T))=$ Ta. Consequently, $r(a) \notin \sigma(T a)$.

It is not immediately clear whether $\sigma(T a)$ in the previous result can be replaced by $\omega_{T}(a)$. However, under the additional assumption that $T$ has either closed range or satisfies the Riesz property, the replacement is possible. Under the first assumption the statement is true by ([19], Theorem 3). If we 
assume the second condition, then, since $r(a) \mathbf{1}-a+\mathrm{N}(T) \in(A / \mathrm{N}(T))^{-1}$ whenever $r(a) \notin \omega_{T}^{L}(a)$ by Lemma 6.2.2, we have that $r(a) \notin \sigma(\pi a)$ and hence $r(a) \notin \omega_{\pi}(a)$ in view of Theorem 1.5.8, where $\pi: A \rightarrow A / \mathrm{N}(T)$. It then follows that $r(a) \mathbf{1}-a \in \mathcal{W}_{\pi}=A^{-1}+\mathrm{N}(\pi)=A^{-1}+\mathrm{N}(T)=\mathcal{W}_{T}$, so that $r(a) \notin \omega_{T}(a)$.

Theorem 6.2.3 or 6.2.4 applied to $\mathcal{L}^{r}(E)$ yields the following:

Corollary 6.2.5. If $T$ is a positive operator on $E$ satisfying $r(T) \in \omega_{\pi_{r}}\left(T, \mathcal{L}^{r}(E)\right)$, then $r(T) \in \omega_{\pi_{r}}^{L}\left(T, \mathcal{L}^{r}(E)\right)$.

We mention that the set $\omega_{\pi_{r}}\left(T, \mathcal{L}^{r}(E)\right)$ is referred to as the order Weyl spectrum of $T$ ([9], Definition 4.1).

We now focus our attention on the following problem: if $a$ is a positive element, under which conditions do we have that $\omega_{T}(a) \subseteq \omega_{T}^{L}(a)$ ? In his papers ([3], remark at the end of p.384) and ([4], (a) and (b), p.17 and Theorem 23), Alekhno identified certain positive operators (and certain types of Banach lattices) for which the inclusion holds. In particular, following the discussion in Section 5 of [4] (with focus on Theorem 23), we have that every positive operator $T$ on either an $A L$ - or an $A M$-space (with unit) satisfies $\omega_{\pi}(T) \subseteq \omega_{\pi}^{L}(T)$. If, in addition, $E$ is Dedekind complete, then (as pointed out several times) $\mathcal{L}(E)=\mathcal{L}^{r}(E)$ and $\mathcal{K}(E)=\mathcal{K}^{r}(E)$ - recall the remark following Example 1.9.4 and Lemma 1.6.9. Most importantly, in view of ([27], Theorem 2.8), we have that the spectral radius function in $\left(\mathcal{L}^{r}(E) / \mathcal{K}^{r}(E), \pi_{r} K\right)$, where $K$ is the cone of positive operators on $E$, is weakly monotone. Though not a generalization of the above-mentioned fact by Alekhno, we have the following result for Banach algebra homomorphisms $T$ such that the spectral radius function in $(A / \overline{\mathrm{N}(T)}, \pi C)$ is weakly monotone.

Proposition 6.2.6. Let $(A, C)$ be an $O B A$ and $B$ be a commutative semisimple Banach algebra. If $T: A \rightarrow B$ is a Banach algebra homomorphism with closed range satisfying the Riesz property such that the spectral radius function in $(A / \overline{\mathrm{N}(T)}, \pi C)$ is weakly monotone, then $\omega_{T}(a) \subseteq \omega_{T}^{L}(a)$ for all $a \in C$.

Proof. Let $a \in C$ and suppose that $\lambda \notin \omega_{T}^{L}(a)$. Then there exists $c \in C$ such that $c \leq b$ for some $b \in \mathrm{N}(T), c \leq a$ and $\lambda \notin \sigma(a-c)$. Since the spectral radius function in $(A / \overline{\mathrm{N}(T)}, \pi C)$ is weakly monotone, we have that $r(T c)=r(\pi c) \leq r(\pi b)=0$ in view of Corollary 2.1.4, and hence $T c \in$ 
$\mathrm{QN}(B)=\operatorname{Rad}(B)=\{0\}$ by assumption. Consequently, $c \in \mathrm{N}(T)$, and thus $\lambda \notin \omega_{T}(a)$ (in fact, $\lambda \notin \omega_{T}^{+}(a)$ in light of Corollary 4.1.10). We have shown that $\omega_{T}(a) \subseteq \omega_{T}^{L}(a)$.

Some remarks: It is interesting to note that the condition " $T$ has closed range and satisfies the Riesz property" in Proposition 6.2.6 can be replaced by " $T$ has the strong Riesz property" (substitute Corollary 2.1.4 with (5.4.4)), " $T$ is bounded and has closed range" (replace Corollary 2.1.4 by Corollary 2.1.3) and " $T$ is bounded and satisfies the Riesz property". Under the latter assumption, there is inequality $r(T c) \leq r(\pi c)$ as $\eta \sigma(T c) \subseteq \eta \omega_{T}(c)=$ $\eta \omega_{\pi}(c)=\eta \sigma(\pi c)$ by (1.5.6) and Theorems 1.5.7 and 1.5.8.

In ([3], p.384) Alekhno remarked that, if $E$ and its order dual $E^{\prime}$ both have order continuous norms, then $\omega_{\pi}^{-}(T)=\omega_{\pi}^{L}(T)$ for all $0 \leq T \in \mathcal{L}(E)$. As mentioned in ([34], p.499) (see also ([7], Theorem 16.20)), under these assumptions on $E$, the algebra cone $\pi K$, where $K:=\left\{T \in \mathcal{L}(E): T E_{+} \subseteq E_{+}\right\}$, is proper in $\mathcal{L}(E) / \mathcal{K}(E)$. Next, we investigate arbitrary Banach algebra homomorphisms $T: A \rightarrow B$, where $(A, C)$ is an OBA, with the property that $T C$ is a proper algebra cone in $B$.

We start our discussion with the following more general version of Theorem 6.1 in [34]. We point out that, to establish this result, a similar idea as in the proof of ([34], Theorem 6.1) is used.

Lemma 6.2.7. Let $(A, C)$ be an $O B A$ and $T: A \rightarrow B$ be a Banach algebra homomorphism. The following two conditions are equivalent:

(i) if $0 \leq a \leq b$ relative to $C$ and $b \in \mathrm{N}(T)$, then $a \in \mathrm{N}(T)$;

(ii) the algebra cone $\mathrm{TC}$ is proper in $B$.

Proof. (i) $\Rightarrow$ (ii). Suppose that the conditions $0 \leq a \leq b$ relative to $C$ and $b \in \mathrm{N}(T)$ imply that $a \in \mathrm{N}(T)$. Let $c \in T C \cap-T C$. Then there exist $c_{1}, c_{2} \in C$ such that $c=T c_{1}=-T c_{2}$, and hence $c_{1}+c_{2} \in \mathrm{N}(T)$. Since $0 \leq c_{1} \leq c_{1}+c_{2}$, we have that $c=T c_{1}=0$ by assumption. Hence $T C$ is a proper algebra cone.

(ii) $\Rightarrow$ (i). Suppose that TC is a proper algebra cone in $B$ and that $a \in A$ and $b \in \mathrm{N}(T)$ are such that $0 \leq a \leq b$ relative to $C$. It then follows that $-T a=T(b-a) \in T C$, so that $T a \in T C \cap-T C=\{0\}$ by assumption, and hence $a \in \mathrm{N}(T)$. 
We are now in a position to present a generalization of the remark by Alekhno preceding Lemma 6.2.7.

Proposition 6.2.8. Let $(A, C)$ be an $O B A$ and $T: A \rightarrow B$ be a Banach algebra homomorphism such that $T C$ is a proper algebra cone in $B$. If $a \in C$, then $\omega_{T}^{-}(a)=$ $\omega_{T}^{L}(a)$.

Proof. It suffices to show that $\omega_{T}^{-}(a) \subseteq \omega_{T}^{L}(a)$. Suppose that $\lambda \notin \omega_{T}^{L}(a)$. Then there exists $c \in C$ such that $c \leq b$ for some $b \in \mathrm{N}(T), c \leq a$ and $\lambda \notin$ $\sigma(a-c)$. From Lemma 6.2.7 we have that $c \in \mathrm{N}(T)$, and hence $\lambda \notin \omega_{T}^{-}(a)$ by definition. This completes the proof.

We mention that in Examples 3.2.10 to 3.2.12 the condition "TC is a proper algebra cone" occurs.

Remark 6.2.9. It is worth pointing out that, under the conditions as stipulated in either Proposition 6.2.6 or Proposition 6.2.8, $\omega_{T}^{L}(a) \neq \varnothing$.

Corollary 6.2.10. Let $(A, C)$ be an $O B A$ and $T: A \rightarrow B$ be a Banach algebra homomorphism such that $T C$ is a proper algebra cone in $B$. If $a \in C$, then $\omega_{T}(a) \subseteq$ $\omega_{T}^{L}(a)$, and hence $\sigma(T a) \subseteq \omega_{T}(a) \subseteq \omega_{T}^{L}(a)=\omega_{T}^{-}(a) \subseteq \sigma(a)$.

If, in addition, $T$ satisfies the Riesz property, then

$$
\sigma(T a) \subseteq \omega_{T}(a) \subseteq \omega_{T}^{+}(a) \subseteq \omega_{T}^{L}(a)=\omega_{T}^{-}(a) \subseteq \sigma(a)
$$

Proof. For $a \in C$, the inclusion $\omega_{T}(a) \subseteq \omega_{T}^{L}(a)$ follows from Propositions 6.1.2 and 6.2.8. By recalling Proposition 6.1.2 and (6.1.4) (in the case where $T$ has the Riesz property), the rest of the result follows.

Note that, even under the assumption "TC is a proper algebra cone in $B$ ", the inclusion $\omega_{T}(a) \subseteq \omega_{T}^{L}(a)$ is strict in general (see Example 6.1.6). 


\section{Conclusion}

In this dissertation we illustrated how a recent discovery in the context of bounded linear operators on Banach lattices has led us to link the positivity and Fredholm theory in Banach algebras equipped with a partial ordering.

The first aim of this thesis was to develop the theory of upper Weyl and upper Browder elements in arbitrary OBAs (Chapter 3). Among other results we showed that, although the classes of Weyl and upper Weyl elements generally do not coincide (Example 3.1.8), relative to some Banach algebra homomorphisms they do (Theorem 3.2.6). This result generalized Example 3.1.3 and can be viewed as a generalization of Alekhno's result (Example 3.1.2) in the case where the Banach lattice is either $A L$ or $A M$.

Our second focus was the study of two new spectra for an element of an OBA (Chapter 4) - these spectra have, as underlying sets, the sets of upper Weyl and upper Browder elements, respectively. In particular, we identified in Theorem 4.3.2 elements whose connected hulls of their Fredholm, Browder, Weyl and upper Weyl spectra coincide and offered an example that illustrated that the connected hull of the upper Browder spectrum cannot in general be added to this list (Example 4.3.3). Consequently, a central problem in this thesis was the following:

Problem: Given that the spectral radius of a positive element is outside its Fredholm spectrum, what conditions suffice for it to be outside the upper Browder spectrum of the element?

As far as we know, this question has not been investigated in the operatorcontext. Throughout Chapter 5 we provided several results pertaining to the above-stated problem - Corollary 5.2.11, Theorem 5.3.3, Proposition 5.3.5 and Corollary 5.5.5 (see also Corollary 6.1.9). In some cases we were only able to obtain results of a weaker form - Theorem 5.3.6, Theorem 5.5.4 and Corollary 5.5.9. Furthermore, in Section 5.4, we showed that the results of Section 5.3 (and of the chapters that follow) can be strengthened by weakening the assumption that the Banach algebra homomorphism $T$ has both 
closed range and the Riesz property to the condition that $T$ has the strong Riesz property.

Finally, we studied in Chapter 6 natural generalizations of the lower Weyl and Lozanovsky essential spectra for a positive bounded linear operator on a Banach lattice in an OBA-context. Specifically, in Section 6.1, a similar problem to that of the aforementioned problem was studied; replacing "upper Browder spectrum" by "lower Weyl spectrum". The main results connected to this problem are contained in Proposition 6.1.8, Theorem 6.1.10 and Corollary 6.1.12. Subsequently, in Section 6.2 we provided some answers (all of which took place in an OBA setting) to the question of when the Weyl spectrum is contained in the Lozanovsky spectrum (cf. Proposition 6.2.6, Corollary 6.2.10).

Some open ends: Although none of our results in either Section 5.3 or Section 5.5 suggests that all positive operators on arbitrary Banach lattices have the upper Browder spectrum property, neither do we have a counterexample. However, we point out that the construction (if possible) of a positive operator on some Banach lattice which does not have the upper Browder spectrum property would answer a number of unsolved problems raised within this dissertation. We mention a few: First the example would confirm that the identity $\mathcal{B}_{\pi}=\mathcal{B}_{\pi}^{+}$does not hold in general. Secondly, it would imply that the commutativity assumption in Proposition 5.3.5 could not in general be dropped and, lastly, that $\beta_{T}^{+}(a u)$ in Theorem 5.3.6 could not be replaced by $\beta_{T}^{+}(a)$ in general.

The question of whether the sets of Weyl and upper Weyl elements coincide whenever a Banach algebra homomorphism $T$ has the Riesz property is still unanswered. An example of such $T$ relative to which the sets of Weyl and upper Weyl elements do not coincide would indicate that our assumption about the span-condition in Theorem 3.2.6(a) is generally essential.

To conclude, we mention that a number of results in this work involve weak monotonicity assumptions on the spectral radius (see, for instance, Proposition 1.9.22, Theorem 5.5.4 and Lemma 6.2.2). It is a natural question as to whether we can drop this assumption in general. If this were possible, then these results would potentially be applicable to operators which are not regular. 


\section{List of references}

[1] Y. A. Abramovich. Some new characterizations of $A M$-spaces. (In Russian). An. Univ. Craiova Mat. Fiz.-Chim., 6:15-26, 1978.

[2] Y. A. Abramovich and C. D. Aliprantis. An invitation to operator theory, volume 50. Grad. Stud. Math., Amer. Math. Soc., Providence, Rhode Island, 2002.

[3] E. A. Alekhno. Some properties of essential spectra of a positive operator. Positivity, 11(3):375-386, 2007.

[4] E. A. Alekhno. Some properties of essential spectra of a positive operator. II. Positivity, 13(1):3-20, 2009.

[5] E. A. Alekhno. The lower Weyl spectrum of a positive operator. Integral Equations Operator Theory, 67(3):301-326, 2010.

[6] E. A. Alekhno. The irreducibility in ordered Banach algebras. Positivity, 16(1):143-176, 2012.

[7] C. D. Aliprantis and O. Burkinshaw. Positive operators. Academic Press, Orlando, 1985.

[8] W. Arendt. On the $o$-spectrum of regular operators and the spectrum of measures. Math. Z., 178(2):271-287, 1981.

[9] W. Arendt and A. R. Sourour. Perturbation of regular operators and the order essential spectrum. Indag. Math., 48(2):109-122, 1986.

[10] B. Aupetit. A Primer on spectral theory. Springer-Verlag, New York, 1991.

[11] J. Banasiak and L. Arlotti. Perturbations of positive semigroups with applications. Springer-Verlag, London, 2006. 
[12] B. A. Barnes, G. J. Murphy, M. R. F. Smyth, and T. T. West. Riesz and Fredholm theory in Banach algebras. Research Notes in Mathematics, 67. Pitman, Boston, 1982.

[13] S. K. Berberian. The Weyl spectrum of an operator. Indiana Univ. Math. J., 20(6):529-544, 1970.

[14] F. F. Bonsall and J. Duncan. Complete normed algebras. Ergebnisse der Mathematik und ihrer Grenzgebiete, 80. Springer-Verlag, New YorkHeidelberg, 1973.

[15] G. Braatvedt, R. Brits, and H. Raubenheimer. Gelfand-Hille type theorems in ordered Banach algebras. Positivity, 13(1):39-50, 2009.

[16] J. B. Conway. Functions of one complex variable. Graduate Texts in Mathematics, 11. Springer-Verlag, New York, second edition, 1978.

[17] J. B. Conway. A course in functional analysis. Graduate Texts in Mathematics, 96. Springer-Verlag, New York, 1985.

[18] J. J. Grobler and H. Raubenheimer. Spectral properties of elements in different Banach algebras. Glasgow Math. J., 33(1):11-20, 1991.

[19] R. E. Harte. The exponential spectrum in Banach algebras. Proc. Amer. Math. Soc., 58(1):114-118, 1976.

[20] R. E. Harte. Fredholm theory relative to a Banach algebra homomorphism. Math. Z., 179(3):431-436, 1982.

[21] R. E. Harte. Fredholm, Weyl and Browder theory. Proc. Roy. Irish Acad. Sect. A, 85(2):151-176, 1985.

[22] J. J. Koliha. A generalized Drazin inverse. Glasgow Math. J., 38(3):367381, 1996.

[23] V. Kordula and V. Müller. On the axiomatic theory of spectrum. Studia Math., 119(2):109-128, 1996.

[24] E. Kreyszig. Introductory Functional Analysis with applications. John Wiley and Sons, New York, 1989.

[25] A. Lebow and M. Schechter. Semigroups of operators and measures of noncompactness. J. Funct. Anal., 7:1-26, 1971. 
[26] L. Lindeboom and H. Raubenheimer. On regularities and Fredholm theory. Czechoslovak Math. J., 52(127)(3):565-574, 2002.

[27] J. Martinez and J. M. Mazón. Quasi-compactness of dominated positive operators and $C_{0}$-semigroups. Math. Z., 207(1):109-120, 1991.

[28] H. du T. Mouton, S. Mouton, and H. Raubenheimer. Ruston elements and Fredholm theory relative to arbitrary homomorphisms. Quaest. Math., 34(3):341-359, 2011.

[29] H. du T. Mouton and H. Raubenheimer. More on Fredholm theory relative to a Banach algebra homomorphism. Proc. Roy. Irish Acad. Sect. A, 93(1):17-25, 1993.

[30] S. Mouton. Convergence properties of positive elements in Banach algebras. Math. Proc. R. Ir. Acad., 102A(2):149-162, 2002.

[31] S. Mouton. A spectral problem in ordered Banach algebras. Bull. Austral. Math. Soc., 67(1):131-144, 2003.

[32] S. Mouton (née Rode) and H. Raubenheimer. More spectral theory in ordered Banach algebras. Positivity, 1(4):305-317, 1997.

[33] V. Müller. Axiomatic theory of spectrum III - Semiregularities. Studia Math., 142(2):159-169, 2000.

[34] H. Raubenheimer and S. Rode. Cones in Banach algebras. Indag. Math. (N.S.), 7(4):489-502, 1996.

[35] H. H. Schaefer. Banach lattices and positive operators. Die Grundlehren der mathematischen Wissenschaften, 215. Springer-Verlag, New YorkHeidelberg, 1974.

[36] H. H. Schaefer. On the $o$-spectrum of order bounded operators. Math. Z., 154(1):79-84, 1977.

[37] S. Č. Živković-Zlatanović, D. S. Djordjević, and R. E. Harte. Ruston, Riesz and perturbation classes. J. Math. Anal. Appl., 389(2):871-886, 2012. 


\section{Index}

$A L$-space, 15

AM-space, 15

$A M$-space with unit, 15

$K B$-space, 15

T-invariant, 14

T-invariant chain, 23

a-invariant, 23

$a$-invariant chain, 23

$f$-pole, 26

algebra, 1

Banach, 2

commutative, 1

complex, 1

disc, 2

real, 1

semisimple, 3

simple, 75

unital, 2

algebra cone, 16

generating, 17

inverse-closed, 18

normal, 17

proper, 17

almost invertible Fredholm element,

11

band, 14

projection, 14 block, 24

Browder element, 11

center of Banach lattice, 82

commutant, 1

commute, 1

connected hull, 5

Dedekind complete vector lattice, 14

disjoint complement, 14

disjunctive product, 25

double commutant, 2

Fredholm element, 11

Frobenius normal form, 26

generalized Drazin inverse, 8 generalized Drazin invertible, 7

holomorphic functional calculus, 8 homomorphism, 3

Banach algebra, 3

homomorphism induced by composition, 37

ideal, 3

inessential, 10

order, 14

infimum of an OBA elment, 20

inverse, 2 
invertible, 2

irreducible element, 23

irreducible w.r.t. an order idempotent, 23

isomorphic, 3

isomorphism, 3

lattice

Banach, 14

complex Banach, 15

normed vector, 14

vector, 13

lower semi-regularity, 56

lower Weyl spectrum property, 95

modulus, 14

negative element, 17

negative part, 14

operator

r-compact, 15

band irreducible, 15

order continuous, 14

positive, 14

regular, 15

spectrally order continuous, 24

order continuous element, 23

order continuous norm, 14

order idempotent, 22

order projection, 22

order-bounded above, 20

ordered Banach algebra (OBA), 17

Dedekind complete, 21

pole, 10

positive cone, 14

positive element, 17

positive part, 14 quasi-invertible, 5

quasi-product, 5

quasinilpotent, 7

radical, 3

regular norm ( $r$-norm), 15

regularity, 56

resolvent, 7

resolvent equation, 7

resolvent identity, 7

resolvent set, 5

Riesz element, 10

Riesz point of spectrum, 10

Riesz property, 10

spectral block, 24

spectral idempotent, 9

spectral mapping theorem, 8

spectral radius, 6

spectral radius monotone, 19

spectral radius preserving, 6

spectral radius weakly monotone,

19

spectrally order continuous element, 24

spectrum, 4

$o-, 69$

almost invertible Fredholm, 13

Browder, 13

essential, 69

Fredholm, 12

lower Weyl, 93

Lozanovsky, 98

Lozanovsky essential, 98

order essential, 69

order Weyl, 101

upper Browder, 59 
upper Weyl, 59

Weyl, 12

spectrum preserving, 5

strong Riesz property, 84

supremum of an OBA element, 20

unitization, 5

upper Browder element, 36

upper Browder spectrum property,

74

upper semi-regularity, 57

upper Weyl element, 36

Weyl element, 11 